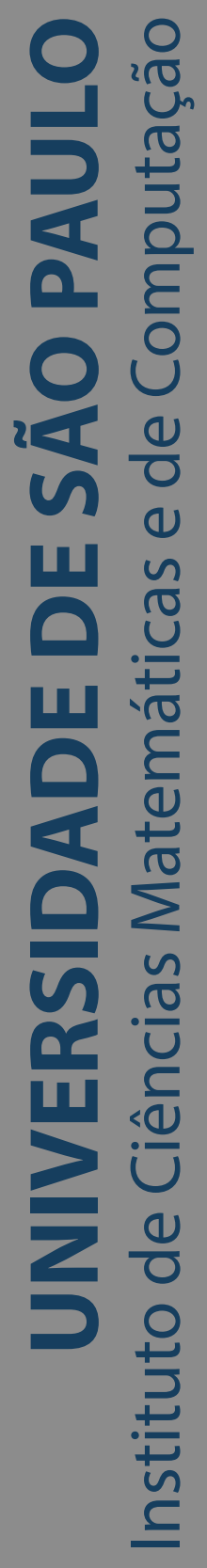

\title{
Self-organization map in complex network
}

\section{Mayra Mercedes Zegarra Pimenta}

Tese de Doutorado do Programa de Pós-Graduação em Ciências de Computação e Matemática Computacional (PPG-CCMC) 

SERVIÇO DE PÓS-GRADUAÇÃO DO ICMC-USP

Data de Depósito:

Assinatura:

Mayra Mercedes Zegarra Pimenta

\section{Self-organization map in complex network}

Doctoral dissertation submitted to the Institute of Mathematics and Computer Sciences - ICMC-USP, in partial fulfillment of the requirements for the degree of the Doctorate Program in Computer Science and Computational Mathematics. FINAL VERSION

Concentration Area: Computer Science and Computational Mathematics

Advisor: Prof. Dr. Francisco Aparecido Rodrigues

USP - São Carlos

August 2018 
Ficha catalográfica elaborada pela Biblioteca Prof. Achille Bassi e Seção Técnica de Informática, ICMC/USP, com os dados inseridos pelo(a) autor(a)

Zegarra Pimenta, Mayra Mercedes

Z44s Self- organization map in complex network /

Mayra Mercedes Zegarra Pimenta; orientador

Francisco Aparecido Rodrigues. -- São Carlos, 2018. $100 \mathrm{p}$.

Tese (Doutorado - Programa de Pós-Graduação em Ciências de Computação e Matemática Computacional) Instituto de Ciências Matemáticas e de Computação, Universidade de São Paulo, 2018.

1. Complex network. 2. Neural networks. 3 . Cllustering algorithm. 4. Self-organization map. 5. Classification. I. Rodrigues, Francisco Aparecido, orient. II. Título.

Bibliotecários responsáveis pela estrutura de catalogação da publicação de acordo com a AACR2: Gláucia Maria Saia Cristianini - CRB - 8/4938 Juliana de Souza Moraes - CRB - 8/6176 
Mayra Mercedes Zegarra Pimenta

\title{
Mapas organizativos em redes complexas
}

\author{
Tese apresentada ao Instituto de Ciências \\ Matemáticas e de Computação - ICMC-USP, \\ como parte dos requisitos para obtenção do título \\ de Doutora em Ciências - Ciências de Computação e \\ Matemática Computacional. VERSÃO REVISADA \\ Área de Concentração: Ciências de Computação e \\ Matemática Computacional \\ Orientador: Prof. Dr. Francisco Aparecido Rodrigues
}



This work is dedicated to God for allowing me to fulfill a desire of my heart and is also dedicated to my husband. Thank you for your unconditional support. 

In the first place, I want to thank God, who gave me this opportunity, who sustained me and strengthened me. I am deeply thankful to my family and my husband who gave me his support with prayers and words of encouragement. Thank you for believing in me.

I would like to thank Prof. Francisco Rodrigues for supervising this Ph.D. and for introducing me to the world of complex networks. I am very grateful to Prof. Luciano da Fontoura Costa for giving me the opportunity to work and learn from him. I am also very grateful to Prof. Cesar Comin for his pertinent comments, suggestions about the work developed in this thesis.

I want to thank my laboratory colleagues from the Computer Department and the Institute of Physics, Didier Vega Oliveros, Guilherme Ferraz de Arruda, Thomas Peron, Filipi Nascimento for the opportunity to exchange ideas and consult my doubts.

I also want to thank ICMC's friends for the good times shared. I acknowledge CAPES for the financial support. 

"They were all trying to frighten us, thinking, Their hands will get too weak for the work, and it will not be completed." But I prayed, Now strengthen my hands." (Nehemiah 6:9) 



\section{ABSTRACT}

PIMENTA, M. M. Z. Self-organization map in complex network. 2018. 100 p. Tese (Doutorado em Ciências - Ciências de Computação e Matemática Computacional) - Instituto de Ciências Matemáticas e de Computação, Universidade de São Paulo, São Carlos - SP, 2018.

The Self-Organization Map (SOM) is an artificial neural network that was proposed as a tool for exploratory analysis in large dimensionality data sets, being used efficiently for data mining. One of the main topics of research in this area is related to data clustering applications. Several algorithms have been developed to perform clustering in data sets. However, the accuracy of these algorithms is data depending. This thesis is mainly dedicated to the investigation of the SOM from two different approaches: (i) data mining and (ii) complex networks. From the data mining point of view, we analyzed how the performance of the algorithm is related to the distribution of data properties. It was verified the accuracy of the algorithm based on the configuration of the parameters. Likewise, this thesis shows a comparative analysis between the SOM network and other clustering methods. The results revealed that in random configuration of parameters the SOM algorithm tends to improve its acuracy when the number of classes is small. It was also observed that when considering the default configurations of the adopted methods, the spectral approach usually outperformed the other clustering algorithms. Regarding the complex networks approach, we observed that the network structure has a fundamental influence of the algorithm accuracy. We evaluated the cases at short and middle learning time scales and three different datasets. Furthermore, we show how different topologies also affect the self-organization of the topographic map of SOM network. The self-organization of the network was studied through the partitioning of the map in groups or communities. It was used four topological measures to quantify the structure of the groups such as: modularity, number of elements per group, number of groups per map, size of the largest group in three network models. In small-world (SW) networks, the groups become denser as time increases. An opposite behavior is found in the assortative networks. Finally, we verified that if some perturbation is included in the system, like a rewiring in a SW network and the deactivation model, the system cannot be organized again. Our results enable a better understanding of SOM in terms of parameters and network structure.

Keywords: Self-organization map, Complex networks, Clustering algorithm. 



\section{RESUMO}

PIMENTA, M. M. Z. Mapas organizativos em redes complexas. 2018. 100 p. Tese (Doutorado em Ciências - Ciências de Computação e Matemática Computacional) - Instituto de Ciências Matemáticas e de Computação, Universidade de São Paulo, São Carlos - SP, 2018.

Um Mapa Auto-organizativo (da sigla SOM, Self-organized map, em inglês) é uma rede neural artificial que foi proposta como uma ferramenta para análise exploratória em conjuntos de dados de grande dimensionalidade, sendo utilizada de forma eficiente na mineração de dados. Um dos principais tópicos de pesquisa nesta área está relacionado com as aplicações de agrupamento de dados. Vários algoritmos foram desenvolvidos para realizar agrupamento de dados, tendo cada um destes algoritmos uma acurácia específica para determinados tipos de dados. Esta tese tem por objetivo principal analisar a rede SOM a partir de duas abordagens diferentes: mineração de dados e redes complexas. Pela abordagem de mineração de dados, analisou-se como o desempenho do algoritmo está relacionado à distribuição ou características dos dados. Verificou-se a acurácia do algoritmo com base na configuração dos parâmetros. Da mesma forma, esta tese mostra uma análise comparativa entre a rede SOM e outros métodos de agrupamento. Os resultados revelaram que o uso de valores aleatórios nos parâmetros de configuração do algoritmo SOM tende a melhorar sua acurácia quando o número de classes é baixo. Observou-se também que, ao considerar as configurações padrão dos métodos adotados, a abordagem espectral usualmente superou os demais algoritmos de agrupamento. Pela abordagem de redes complexas, esta tese mostra que, se considerarmos outro tipo de topologia de rede, além do modelo regular geralmente utilizado, haverá um impacto na acurácia da rede. Esta tese mostra que o impacto na acurácia é geralmente observado em escalas de tempo de aprendizado curto e médio. Esse comportamento foi observado usando três conjuntos de dados diferentes. Além disso, esta tese mostra como diferentes topologias também afetam a auto-organização do mapa topográfico da rede SOM. A auto-organização da rede foi estudada por meio do particionamento do mapa em grupos ou comunidades. Foram utilizadas quatro medidas topológicas para quantificar a estrutura dos grupos em três modelos distintos de rede: modularidade, número de elementos por grupo, número de grupos por mapa, tamanho do maior grupo. Em redes de pequeno mundo, os grupos se tornam mais densos à medida que o tempo aumenta. Um comportamento oposto a isso é encontrado nas redes assortativas. Apesar da modularidade, tem um alto valor em ambos os casos.

Palavras-chave: SOM, Redes complexas, Algoritmos de agrupamento. 

Figure $1-$ example of an undirected network $\ldots \ldots \ldots . \ldots \ldots$

Figure $2-$ Degree distribution of deactivation model . . . . . . . . . . . . 36

Figure 3 - Figure adapted from (KLEMM; EGUÍLUZ, 2002) . . . . . . . . . . 36

Figure 4 - BA network with the degree correlated modified by the approach. (a) BA assortative com $r=0.32$ e (b) BA dissortative com $r=-0.47 \ldots \ldots . . . .37$

Figure 5 - Schematic Diagram of SOM . . . . . . . . . . . . 39

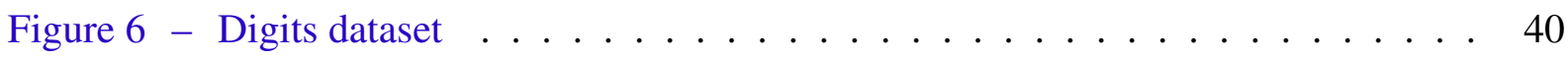

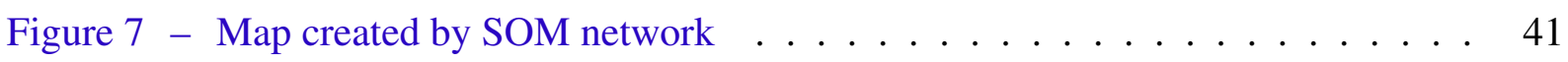

Figure 9 - Average performance of the seven considered clustering algorithms according to the number of features in the dataset. All datasets described in Section 3.3 were used for evaluation. The averages were calculated separately for datasets containing 2, 10 and 50 features. The considered performance indexes are (a) adjusted Rand, (b) Jaccard, (c) normalized mutual information and (d) Fowlkes Mallows. . . . . . . . . . . . . . . . . . . . . . . . 59

Figure 10 - Average performance of the seven considered clustering algorithms according to the number of objects per class in the dataset. All datasets described in Section 3.3 were used for evaluation. The averages were calculated separately for datasets containing 5, 50 and 100 objects per class. The considered performance indexes are (a) adjusted Rand, (b) Jaccard, (c) normalized mutual information and (d) Fowlkes Mallows. . . . . . . . . . . . . . 60

Figure 11 - Distribution of ARI values obtained for the random sampling of the SOM parameters. The algorithm was applied to dataset DB10C10F and DB10C2F, and 500 sets of parameters were drawn. . . . . . . . . . . . . 65

Figure 12 - Dataset: From top to bottom correspond to the EMNIST, MNIST and musical symbols dataset. . . . . . . . . . . . . . . . . . . 73 
Figure 13 - Error rate as a function of the number of learning steps for different datasets and network models. The first, second and third rows of plots correspond, respectively, to the digits and musical symbols and letters dataset. Each column correspond to a model network. From left to right correspond to SW, deactivation model and degree correlated networks. The first and second columns correspond to a map size of $50 \times 50$. In the third column a map size of $35 \times 35$ where used. Figures $13 \mathrm{a}, 13 \mathrm{~d}$ and $13 \mathrm{~g}$ show the results for the small-world model. The results for the deactivation model are shown in Figures 13b, 13e and 13h. Figures 13c, 13f and 13i show the results for the degree-degree correlated networks. In all cases $\langle k\rangle=4$ was used and the

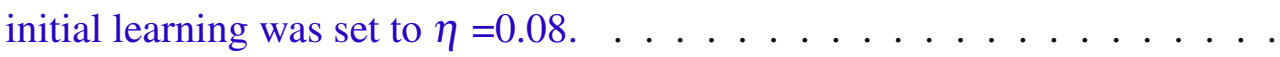

Figure 14 - Error rate as a function of network properties for the digits dataset. The plots from top to bottom correspond to the small-world, degree-degree correlated and deactivation models. Inset plots show the relationship between selected pairs of topological measurements. . . . . . . . . . . . . .

Figure 15 - Error rate as a function of model parameters for the digits dataset. The plots from top to bottom correspond to the small-world, degree-degree correlated and deactivation models. . . . . . . . . . . . . . . . .

Figure 16 - Error rate of the SOM maps as a function of training time. From top to bottom the plots correspond to small-world, deactivation and assortative networks. Map sizes of 20x20, 35x35 and 70x70 were used. . . . . . . . . . . 79

Figure 17 - Example of SOM result for a map of size 10x10 . . . . . . . . . . . . 80

Figure 18 - Average number of neurons per group, $\bar{e}_{\text {groups }}$, and number of groups, $\bar{n}_{\text {groups }}$, as a function of error rates obtained for distinct number of learning steps used when training SOM models. The plots from top to bottom correspond to small-world, deactivation and assortative networks. . . . . . . . . . .

Figure 19 - Size of the largest group as a function of the error rate for different network models. The models are (a) small-world, (b) deactivation and (c) assortative.

Figure 20 - Standard deviation of group sizes as a function of the error rate. The plots from top to bottom correspond to small-world, deactivation and assortative networks. . . . . . . . . . . . . . . . . .

Figure 21 - Modularity as a function of training time for the digits dataset. From top to bottom correspond to small world network, deactivation model and degree correlated networks. Inset plots show the relationship between modularity and error rate. . . . . . . . . . . . . . . . . . . . . 
Table 1 - Average difference of accuracies obtained when clustering algorithms are used with their default configuration of parameters. In general, the spectral algorithm provides the highest accuracy rate among all evaluated methods.

Table 2 - One-parameter analysis performed in DB2C2F and DB10C2F. This analysis is based on the performance (measured through the ARI index) obtained when varying a single parameter of the clustering algorithm while maintaining the others in their default configuration. $\langle S\rangle, \max S, \Delta S$ is associated with the average, standard deviation and maximum difference between the performance obtained when varying a single parameter and the performance obtained for the default parameter values. We also measure $\langle\max A c c\rangle$, the average of best ARI values obtained when varying each parameter, where the average is calculated over all considered datasets. . . . . . . . . . . . . . . .

Table 3 - One-parameter analysis performed in DB2C10F and DB10C10F. This analysis is based on the performance obtained when varying a single parameter, while maintaining the others in their default configuration. $\langle S\rangle$, $\max S, \Delta S$ are associated with the average, standard deviation and maximum difference between the performance obtained when varying a single parameter and the performance obtained for the default parameter values. We also measure $\langle\max A c c\rangle$, the average of best ARI values obtained when varying each parameter, where the average is calculated over all considered datasets. . . . . . . . . . . . . .

Table 4 - Multi-parameter analysis performed in dataset DB2C2F. The p-value represents the probability that the classifier set with a random configuration of parameters outperform the same classifier set with its default parameters. $\langle R\rangle$, $\Delta R$ and $\max R$ represent the average, standard deviation and maximum value of the improvement obtained when random parameters are considered. Column $\langle\max$ ARI $\rangle$ indicates the average of the best accuracies obtained for each dataset. 66

Table 5 - Multi-parameter analysis performed in dataset DB10C2F. The p-value represents the probability that the classifier set with a random configuration of parameters outperform the same classifier set with its default parameters. $\langle R\rangle$, $\Delta R$ and $\max R$ represent the average, standard deviation and maximum value of the improvement obtained when random parameters are considered. Column $\langle\max$ ARI $\rangle$ indicates the average of the best accuracies obtained for each dataset. 66 
Table 6 - Multi-parameter analysis performed in dataset DB2C10F. The p-value represents the probability that the classifier set with a random configuration of parameters outperform the same classifier set with its default parameters. $\langle R\rangle$, $\Delta R$ and $\max R$ represent the average, standard deviation and maximum value of the improvement obtained when random parameters are considered. Column $\langle\max A R I\rangle$ indicates the average of the best accuracies obtained for each dataset. 66

Table 7 - Multi-parameter analysis performed in dataset DB10C10F. The p-value represents the probability that the classifier set with a random configuration of parameters outperform the same classifier set with its default parameters. $\langle R\rangle$, $\Delta R$ and $\max R$ represent the average, standard deviation and maximum value of the improvement obtained when random parameters are considered. Column $\langle$ max ARI $\rangle$ indicates the average of the best accuracies obtained for each dataset. 67

Table 8 - Summary table for the performance of clustering algorithms in datasets $\mathrm{DB} 2 \mathrm{C} 2 \mathrm{~F}$ and $\mathrm{DB} 10 \mathrm{C} 2 \mathrm{~F}$. ARI $I_{\text {def }}$ represents the average accuracy obtained when considering the default parameters of the algorithms. $A R I_{\text {best }}$ represents the average of the best accuracies obtained when varying a single parameter. ARI best $_{r}$ represents the average of the best accuracies obtained when parameters are randomly selected. . . . . . . . . . . . . 68

Table 9 - Summary table for the performance of clustering algorithms in datasets $\mathrm{DB} 2 \mathrm{C} 10 \mathrm{~F}$ and $\mathrm{DB} 10 \mathrm{C} 10 \mathrm{~F} . A R I_{d e f}$ represents the average accuracy obtained when considering the default parameters of the algorithms. ARI best $_{p}$ represents the average of the best accuracies obtained when varying a single parameter. ARI best $_{r}$ represents the average of the best accuracies obtained when parameters are randomly selected. . . . . . . . . . . . . . . 


\section{LIST OF ABBREVIATIONS AND ACRONYMS}

$\begin{array}{ll}\text { ANNs } & \text { Artificial Neural Network } \\ \text { ARI } & \text { Adjusted Rand Index } \\ \text { BA } & \text { Barabási-Albert } \\ \text { BMU } & \text { Best Matching Unit } \\ \text { DB10C10F } & \text { Dataset with 10 classes and 10 features } \\ \text { DB10C2F } & \text { Dataset with 10 classes and 2 feature } \\ \text { DB2C10F } & \text { Dataset with 2 classes and 10 features } \\ \text { DB2C2F } & \text { Dataset with 2 classes and 2 feature } \\ \text { ER } & \text { Erdős Rényi } \\ \text { FM } & \text { Fowlkes Mallows Index } \\ \text { J } & \text { Jaccard Index } \\ \text { NMI } & \text { Normalized Mutual Information } \\ \text { SOM } & \text { Self-Organization map } \\ \text { SW } & \text { Small World }\end{array}$



$N$ - number of vertices

$A-$ Network adjacency matrix

$A_{i j}$ - Elements of line $\mathrm{i}$ and column $\mathrm{j}$ of the adjacency matrix

$k_{i}$ - degree of node $\mathrm{i}$

$P(k)$ — Probability of distribution of degree $\mathrm{k}$

$c c_{i}-$ Clustering coefficient of vertice $\mathrm{i}$

$L$ - average shortest path length

$Q$ - network modularity

$r$ - Assortativity coefficient

$M$ - number of edges in the network

$p$ - Rewiring probability in the SW model

$\mu$ - Connection probability in the range of $[0,1]$

$w(i, j)$ - weight of connection between neuron $\mathrm{i}$ and $\mathrm{j}$

$N_{e}$ - Number of object per class

$F$ - Number of features

$\alpha$ - Separation between the classes

$C$ - number of classes

$\langle S\rangle$ - average value of improvement in performance

$\max S$ - maximum value of improvement in performance

$n_{P}$ - is the cardinality of all possible values taken by the parameter $P$ in our experiments

$\Delta S$ - standar desviation obtained between default parameter and when varying a single parameter 

1.1 Motivation and contextualization . . . . . . . . . . . 26

1.1.1 Analysis of the SOM network from the perspective of complex networks . . . . . . . . . . . . . . . . . . . 26

1.1.2 Sensitivity analysis of SOM in clustering task . . . . . . . . . . 27

$1.2 \quad$ Goals . . . . . . . . . . . . . . . . . . . 28

1.2.1 Topological measurements of complex networks . . . . . . . . . . 28

1.2.2 The modular structure of SOM . . . . . . . . . . . . . . 28

1.2.3 Artificial Dataset . . . . . . . . . . . . . . . 28

$1.3 \quad$ Contributions . . . . . . . . . . . . . . . 28

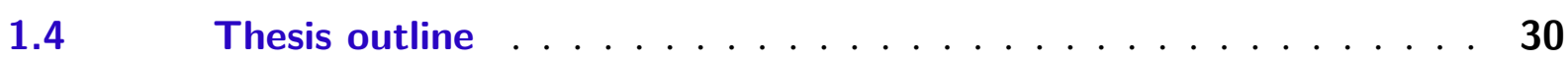

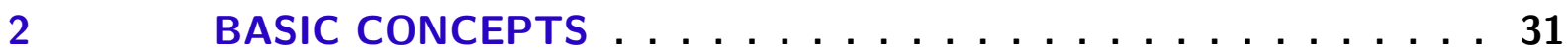

$2.1 \quad$ Complex networks . . . . . . . . . . . . . . . . 31

2.1.1 Terminology and metrics . . . . . . . . . . . . . 31

2.1.2 Global measures . . . . . . . . . . . . . . . . . . 33

2.1.2.1 Modularity . . . . . . . . . . . . . . . 33

2.1.2.2 Assortativity coefficient . . . . . . . . . . . . . 34

2.1.3 Network models . . . . . . . . . . . . . . . . . . 34

2.1.3.1 The Erdös-Rényi model . . . . . . . . . . . . . . . . . 34

2.1.3.2 The Watts-Strogatz model . . . . . . . . . . . . . . . . . 34

2.1.3.3 Barabási-Albert model . . . . . . . . . . . . . . . 35

2.1.3.4 Deactivation model . . . . . . . . . . . . . . 35

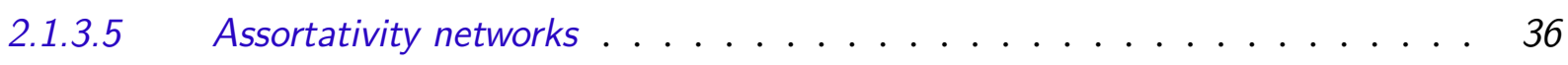

$2.2 \quad$ Artificial neural network . . . . . . . . . . . . 38

2.2.1 Self-organization map . . . . . . . . . . . . . 38

2.2.2 Variations in the parameters . . . . . . . . . . . 41

$2.3 \quad$ Data clustering . . . . . . . . . . . . . . . 41

2.3.1 Taxonomies of clustering of data . . . . . . . . . . . . 43

2.3.2 Hierarchical methods . . . . . . . . . . . . . . . . 44

2.3.3 Partitional methods . . . . . . . . . . . . . . . . . 44

2.3.4 Methods based on density . . . . . . . . . . . . . 45

2.3.5 Methods based on models . . . . . . . . . . . . . . . . . . 45 
2.3.6 Subspace- based methods . . . . . . . . . . . . . . 46

2.3.7 Final remarks . . . . . . . . . . . . . . . . . . . 46

3 ANALYSIS COMPARATIVE OF CLUSTERING ALGORITHM . . . 47

$3.1 \quad$ Background and related works . . . . . . . . . . . . 48

$3.2 \quad$ Package in $\mathbf{R} \ldots \ldots \ldots \ldots \ldots \ldots$

$3.3 \quad$ Method description ................. 51

3.4 Description of the clustering algorithms' parameters . . . . . . 53

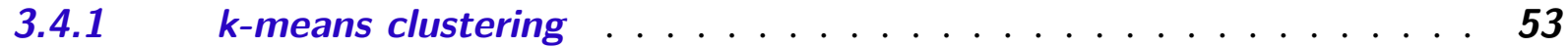

3.4.2 Clustering for large applications (clara) . . . . . . . . . . . . 54

3.4.3 Hierarchical clustering . . . . . . . . . . . . . . . 54

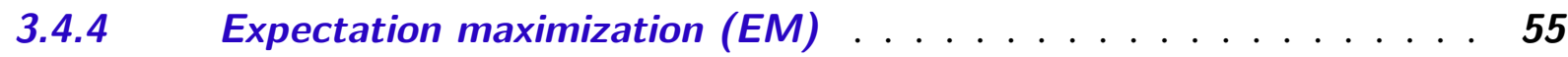

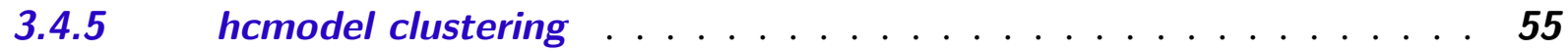

3.4.6 Spectral algorithm . . . . . . . . . . . . . . 56

3.4.7 Subspace algorithm . . . . . . . . . . . . . . . 57

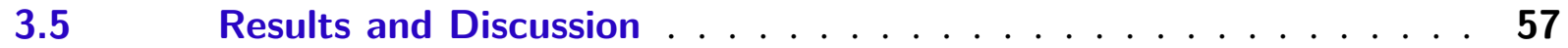

3.5.1 Performance when using default parameters . . . . . . . . . . . . 57

3.5.2 One-dimensional analysis . . . . . . . . . . . . . . . 60

3.5.3 Multi-dimensional analysis . . . . . . . . . . . . . . 63

$3.6 \quad$ Conclusions . . . . . . . . . . . . . . . . . . . 67

4 THE IMPACT OF INTERCONNECTING TOPOLOGIES ON SOM NEURAL NETWORKS . . . . . . . . . . . . . . 71

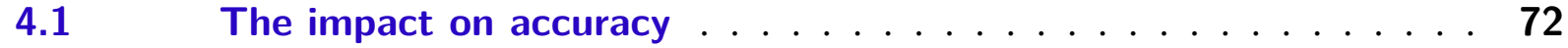

4.1.1 Dataset . . . . . . . . . . . . . . . 72

4.1.2 Analysis and results . . . . . . . . . . . . . . 72

$4.2 \quad$ Analysis of topological properties of map SOM . . . . . . . . 78

$4.3 \quad$ Final remarks . . . . . . . . . . . . . . . . . . 84

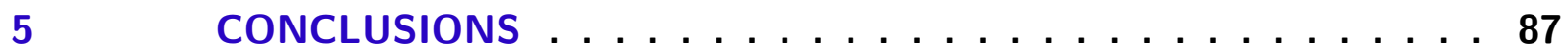

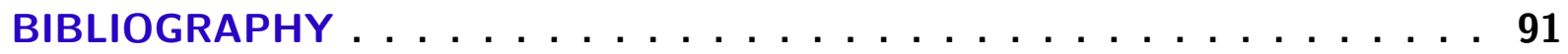


CHAPTER

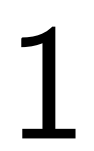

INTRODUCTION

The artificial neuronal networks (ANN) are inspired by the structure and dynamics of biological neural networks. The interest in these neural networks is motivated from the brain remarkable information processing, high parallelism, robustness, fault and noise tolerance. These characteristics enable generalization and learning capabilities (BASHEER; HAJMEER, 2000). ANN have been extensively studied for two main purpose: (1) to better understand biological neuroscience (ARBIB, 2003; RUSSO; HERRMANN; ARCANGELIS, 2014) and (2) to obtain effective methods for machine learning and pattern recognition tasks (BISHOP, 1995), pattern classification (ZHANG, 2000) and forecasting (ZHANG; PATUWO; HU, 1998). As an example of an artificial network that has been used in these two purposes corresponds to the self-organization neural map (SOM) also called Kohonen network.

SOM is an unsupervised artificial neural network proposed by Teuvo Kohonen (KOHONEN, 1982) and based on a competitive learning. It has been extensely used for many domains such as gesture recognition (CARIDAKIS et al., 2010), segmentation, visualization, clustering (TASDEMIR; MERÉNYI, 2009) and classification tasks (CORRIDONI; BIMBO; LANDI, 1996). Currently, SOM is a very useful tool in data analysis (KOHONEN, 2013).

Our interest on the study and analysis of SOM is based on three main goals: (i) to determine how the neuronal interconnection topology influences the learning and accuracy of the obtained neuronal network, (ii) to analyse if the self-organization of topographic map is preserved at different learning time scales when considering different types of topology, (iii) to verify whether the accuracy of SOM network in clustering task is related to the distribution of data probability or to the sensitivity with respect to their parameters configuration.

Regarding the first two goals, we are going to analyse the SOM network from the perspective of complex networks. Recent studies have shown an increasing interesting in the study of real networks made of thousands or millions of vertices, such as social and technological networks (PALLA; BARABÁSI; VICSEK, 2007). Among these studies, there is a particular 
interest in on the influence of network topology on dynamical processes occurring on networks, like synchronization, epidemic spreading and learning tasks (COSTA et al., 2011). Particularly, in this thesis we are interested in study how the network topology affects the performance of SOM structured as complex networks.

Related to our third objective, we will verify how the parameters used in SOM for data clustering influence the results with respect to small changes in the data.

\subsection{Motivation and contextualization}

\subsubsection{Analysis of the SOM network from the perspective of complex networks}

Previous studies on SOM showed the impact of the structure of neural networks on learning processes. Including the following issues:

- Quantification of the topological influence on the retrieval of patterns.

- Quantification of the error rate of classification for a given dataset.

- To study the influence of the removal of certain vertices on the accuracy of the neural network.

Initial studies on the influence of network topology focused on the Hopfield network (HOPFIELD, 1982), also called associative memory networks. This type of network allows storing a set of patterns, such that when an input pattern is presented to the network, the output will be the pattern most similar to the input pattern. In (MCGRAW; MENZINGER, 2003) and (MCGRAW; MENZINGER, 2003) the difference in the pattern recovery rate in network topologies such as scale free (SF), small world networks (SW) and random networks was studied. It was verified that Hopfield networks with small-world properties produce far better results in terms of memory storage than networks with random and regular connectivity.

Another type of network used in this scenario is the multi-layered feed-forward network. In Simard, Nadeau and Kröger (2005) supervised learning with back-propagation in a multilayered feed-forward network with different network architectures, such as small-world and random networks, was studied. It was shown that rewiring on network structure reduces the learning error and learning time when compared to regular and random networks. In (ERKAYMAZ; ÖZER; YUMUŞAK, 2014) the impact of the small-world topology on the performance of a multi-layered feed-forward networks was studied based on two real-life problems and it was shown that small-world networks perform statistically significantly better than regular networks.

Studies specifically related to the SOM in complex networks has not been yet extensively studied in the relation of topological influence in function of classification performance. In (Jiang; 
Berry; Schoenauer, 2007) was compared the performance of SOM neural network whose neighbourhood relationships were defined by a regular, small world and random network. The results show the performance of the network is weakly controlled by its topology at medium and high scales of training. By the other hand at short scales the topology has a bigger impact on performance. Was observed that the same differences can be exploited by evolutionary algorithms. The robustness of the results with respect to noise were also addressed. For instance, Burguillo (2014) explored the use of complex network topologies, like random, scale-free and small-world networks for connecting the neurons within a SOM, which are often applied for time series prediction. Their results suggest that small-world and random networks provide similar results. On the other hand, scale-free topologies do not typically lead to good prediction as a consequence of their heterogeneity. García-Rois and Burguillo (2017) studied the impact of different parameters of the SOM such as number of neurons and learning rate, on the performance when applied to time series prediction considering regular and complex topologies. It was found, for specific parameter configurations, that the small-world and scale-free topologies can present better performance than regular lattices.

Few studies have considered the topological influence of the network and among all those studies that have been done, all of them were limited to the case of classic topologies, such as the small-world and scale-free networks. Aspects such as assortativity and modular structure of topographic maps have not bee analysed yet.

\subsubsection{Sensitivity analysis of SOM in clustering task}

It is well known that machine learning methods play a key role not only in computer science, in areas such as robotics and image processing, but also in medicine (HOWE et al., 2008), ecology (REICHMAN; JONES; SCHILDHAUER, 2011), and many others. Therefore, the development of more efficient algorithms of machine learning or the improvement of algorithms that already exist has become a significant task because it allows to solve several types of real problems. Machine learning methods can be divided into two types: (i) supervised (predictive) and (ii) unsupervised learning (BISHOP et al., 2006; FACELI et al., 2011). Clustering of data is a unsupervised learning, where the goal is to classify objects according to the similarity of their characteristic vectors considering a distance measure. Clustering algorithms involve several parameters, often operate in high dimensional spaces, and have to cope with noisy, incomplete and sampled data. Their performance can vary substantially for different applications and types of data. For such reasons, several different approaches to clustering have been proposed in the literature.

Many works have used SOM networks as a clustering algorithm. In (MO et al., 2017) a self-organizing-map (SOM) based clustering algorithm used to automatically classify colors on printed fabrics, is introduced. The results show that the intricate color patterns can be precisely separated into individual regions representing different colors. In (AHMAD; YUSOF, 2016) it is 
proposed a modification of SOM inspired from pheromone approach in ant colony optimization. The result applied in categorical data show that the modified SOM has produced accurate clustering result and all clusters can clearly be identified.

Although some works have used the SOM as a clustering algorithm, only a few have focused on the sensitivity analysis of the network configuration parameters in the clustering results. To evaluate the sensitivity of the input parameter configuration for each clustering algorithm on the accuracy, we focus on the use of artificial data containing not only distinct number of classes, features, number of objects and separation between classes, but also a predefined correlation and distributions between features. This is a novel approach to generate artificial data and can provide more detailed analysis of cluster evaluation methods.

\subsection{Goals}

The three main goals of this thesis are: First, to study and analyze how different interconnectivity topologies influence the performance of SOM networks. Second, to analyse the topological properties of the topographic map SOM. Third, to evaluate the accuracy of SOM as clustering algorithm, considering different artificial dataset and using different configurations in the parameters. In the following, we present the specific objectives:

\subsubsection{Topological measurements of complex networks}

In this task, the objective is to evaluate the performance of SOM networks and verify which measures, such as clustering coeficient $(C C)$, average path lenght $(L)$ and assortativity, have a greater influence in the results obtained.

\subsubsection{The modular structure of SOM}

Analyse the formation of groups in the topological map of SOM network using for that purpose the measure of modularity. Other topological properties are also considered, such as the number of elements per group, number of groups and the size of the largest group.

\subsubsection{Artificial Dataset}

The purpose of using artificial data is the possibility of obtaining an unlimited number of samples and to systematically change properties such as number of features, number of objects and separation between classes, correlation and distributions between features of a dataset.

\subsection{Contributions}

- How do different topologies affect the rate in neural network SOM? 
To answer it, we will consider small world, deactivation model and degree-degree correlated networks (ARRUDA et al., 2016) which is based on a simulated annealing procedure to create networks with different levels of assortativity. Using three different databases such as digits, musical symbols and letters dataset.

We found that the interconnecting topology has an influence on the performance of the networks at short and middle learning time scales. It was studied how the variation of the level of assortativity in the network model affects the performance of the network. A high level of assortativity implies the creation of small clusters in the network, preventing the update process to be expanded to the other modules of the network, resulting in a decrease in the error rate. In the deactivation model, adding a small amount of long-range connections (10\%) tended to worsen the network performance. In chapter 4 we present these results in details.

- How are the topological properties of topographic map SOM considering different topologies?

To answer this question we must remember that once the training time is over, the input patterns are mapped into the two-dimensional output map of the SOM network depending on the proximity between them. Similar patterns will be located side by side. The output map will be called in the text as topological map. We have analysed the properties of topological maps created by SOM networks considering the above mentioned topologies. In small-world networks when the parameter of rewiring $p=0$, the mean number of elements per group increases with time. This means that the groups begin to be denser and become more defined, which is also corroborated with the measure of modularity, whose obtained value is around 0.7 . However when the parameter $p>0$, the number of elements per group remains the same as in the training step as the time increases, and in turn, the network does not self-organize itself. At short time scale, when the assortativity has high values, the modularity obtained is around of 0.1 , which indicates that the network is disorganized. At middle short time scale the network begins to self-organize, obtaining a modularity of 0.3 approximately. In chapter 4 we present this results in details.

- Sensitivity to the input parameters considering different algorithms of clustering

We evaluated many clustering algorithms considering their performance when using the default parameters, through the variation of a single parameter and the simultaneous variation of all parameters. It was used artificial data generated through a method that allows controlling number of features, number of classes and the variance and correlation distributions among the features. The method to generate this data is also one contribution presented on this work. Regarding the default parameters, the difference in performance of clustering methods was not significant for low-dimensional datasets. Regarding single parameter variations, for datasets containing 10 or more features, most methods could 
be readily improved through changes on selected parameters. With respect to multidimensional analysis for datasets containing ten classes and ten features, the improvement for the multidimensional selection of parameters was significantly lower for almost all the algorithms. In chapter 3 we presented these results in details.

\subsection{Thesis outline}

Next, the text is divided as follows: In Chapter 1, we have contextualized this thesis introducing briefly the objectives, the motivation and the contributions. In Chapter 2 we introduce basic concepts about three main topics of this thesis: complex network, clustering of data and selforganization neural maps. In Chapter 3, we show a comparative analysis of different clustering methods considering a variety of artificial datasets. We quantify the sensitivity of the performance of clustering algorithms with respect to small changes in the dataset. Specially, we observe that default parameters are not always a good alternative to obtain the best accuracy results for the studied algorithms.

In Chapter 4, we study the neural network self-organization map. First, we perform a study of the impact of the accuracy in this type of network considered different topologies and different dataset. Subsequently, we analyse the topological properties of the map generated by the SOM network. We show how the SOM network is self-organized considering the different topologies studied, as training time increases. For this we use the measure of modularity and density intra and inter communities to quantify these differences. In Chapter 5 we present the conclusions regarding the results obtained in this work and future works that can be performed later. 
CHAPTER

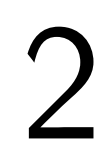

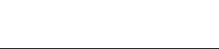

\section{BASIC CONCEPTS}

We shall introduce in this chapter some basic concepts necessary for the understanding of the studies developed in this thesis. Concepts of network science, network metric and network models are presented here. Moreover, we will introduce concepts of artificial neural networks. We will also include concepts and approaches related to data clustering.

\subsection{Complex networks}

\subsubsection{Terminology and metrics}

The organization of complex systems can be represented mathematically as graphs. A graph $G=(N, E)$, consists of two sets: one of nodes and one of edges, $E$. That is, $N$ vertices connected by $M$ edges. The connections of such a graph can be stored in an adjacency matrix $A$, whose elements of line $i$ and column $j$ of the adjacency matrix are defined as $A_{i j}$. $A_{i j}=\left\{\begin{array}{cc}1 & \text { if nodes } \mathrm{i} \text { and } \mathrm{j} \text { are connected by and edge, } \\ 0 & \text { otherwise }\end{array}\right.$

When the adjacency matrix is symmetric $\left(A_{i j}=A_{j i}\right)$ the network is called undirected network. In the case that $A_{i j}=1$, but not necessarily $A_{j i}=1$, we can say that the matrix is not symmetric and we can call this network of directed.

In Figure 1 shows an example of undirected network and its adjacency matrix.

It is necessary to use descriptors of network topology, which are measures of complex networks, to extract useful information about the network structure. Topological measurements have been developed for the characterization, analysis and classification of complex networks (NEWMAN, 2010a). A simple measure is the degree, or connectivity, of a vertex, which is equal to its number of connections. This measure can be calculated in terms of the adjacency matrix $A$ and it 
Figure 1 - example of an undirected network

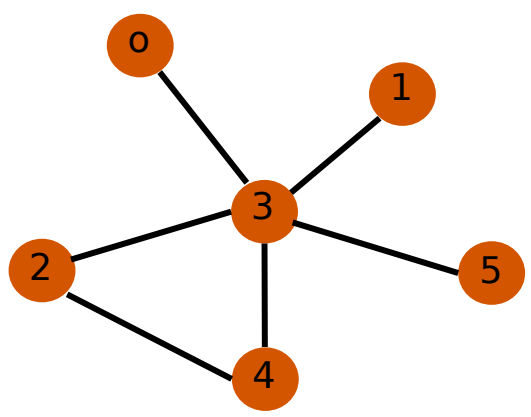

012345

00 \begin{tabular}{llllll|}
0 & 0 & 0 & 1 & 0 & 0 \\
\hline
\end{tabular}

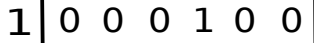

2000001100

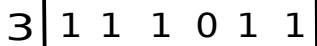

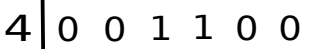

$50 \begin{array}{llllll}0 & 0 & 0 & 1 & 0 & 0\end{array}$

Source: Elaborated by the author.

is expressed as $k_{i}$

$$
k_{i}=\sum_{j=1}^{N} A_{i j}
$$

The network average degree is expressed as,

$$
\langle k\rangle=\frac{1}{N} \sum_{j=1}^{N} k_{i} .
$$

The organization of the connections of a network can be quantified in terms of its degree distribution, $P(k)$, which is defined as the probability that a randomly chosen node has degree $k$. Complex networks can also be characterized in terms of clustering coefficient $(C)$, which is define as the average fraction of pairs of neighbors of a node that are also neighbors of each other (WANG; CHEN, 2003). It can be described as,

$$
C=\frac{3 * \text { number of triangles }}{\text { number of triples }}
$$

where a triangle is defined as a set of three nodes connected together. A triple is a sequence of three nodes $i, j, k$ connected by two edges.

The local clustering coefficient, is defined as $c c_{i}$, is given by the equation

$$
c c_{i}=\frac{2 e_{i}}{k_{i}\left(k_{i}-1\right)}=\frac{\sum_{j=1}^{N} \sum_{m=1}^{N} A_{i j} A_{j m} A_{m i}}{k_{i}\left(k_{i}-1\right)},
$$

where $e_{i}$ represents the number of connections between the neighbors of the vertex $i$.

Shortest paths or geodesic paths $d_{i j}$ represent an important topic of studies in networks, mainly in the analysis of transport and communication. A path between $i$ and $j$ is a sequence of 
vertices and edges (adjacent nodes) that starts at $i$ and ends at $j$, where no vertex is visited more than once (BOCCALETTI et al., 2006). The average shortest path length $L$ is given by,

$$
L=\frac{1}{N(N-1)} \sum_{i \neq j} d_{i j} .
$$

\subsubsection{Global measures}

\subsubsection{Modularity}

In (FORTUNATO, 2010), the communities are defined as groups of vertices which probably share common properties and/or play similar roles within the graph. Another definition is described as a collection of vertices in a subgraph that is densely connected among themselves while being loosely connected to the vertices outside the subgraph (LI et al., 2008).

According to Newman (2006) if the number of edges between two groups is what one would expect by a random choice, so this not constitutes evidence of meaningful community structure. On the other hand, if the number of edges between groups is significantly less or more than that obtained by chance, then the network present community structure.

The importance of the detection of communities is to reveal the hidden structures of networks and to know the properties and relationship of nodes in a graphical network which is not possible from direct observations.

Newman and Girvan (2004) defined a quality function $Q$ to measure the community structure of a network. In equation 2.6 the modularity $\mathrm{Q}$ is expressed as

$$
Q=\frac{1}{2 M} \sum_{i j}\left(A_{i j}-\frac{k_{i} k_{j}}{2 M}\right) \delta_{I_{i}, I_{j}}
$$

where $A_{i j}$ adjacency matrix of elements $i$ e $j, M$ number of connections, $I_{x}$ indicates the index of the community membership of node $x . \delta_{I_{i}, I_{j}}=1$ se $I_{i}=I_{j}$ e $\delta_{I_{i}, I_{j}}=0$ otherwise and $\frac{k_{i} k_{j}}{2 M}$ indicates the expected number of connections between nodes with degree $k_{i}$ and $k_{j}$ if the network were constructed in a uniformly random fashion.

Several algorithms were proposed based on different approaches that allowed to identify a good partition of groups in a graph, such as the algorithms devised to maximize modularity (CLAUSET; NEWMAN; MOORE, 2004; FORTUNATO, 2010) and the method based on the spectrum of adjacency matrix (NEWMAN, 2006). Was recently suggested an alternative global measure of partition called Surprise (ALDECOA; MARÍN, 2013). Surprise evaluates not only the number of links, but also the number of nodes within each community this allows that small communities to be detected.

Although many modularity measures have been proposed, we will use the modularity of the equation 2.6 to evaluate the groups created by the SOM network in the different network models used. 


\subsubsection{Assortativity coefficient}

The assortativity coefficient $r$ is a measure of degree-degree correlation. It quantifies the relationship between the degrees of neighbouring nodes. For instance, a large assortativity coefficient means that a node with large degree tends to connect to nodes also with large degrees $(r=+1)$. It is calculated by taking into account the Pearson correlation coefficient between pairs of edges connected by an edge (NEWMAN, 2003),

$$
r=\frac{\frac{1}{M} \sum_{j>i} k_{i} k_{j} A_{i j}-\left[\frac{1}{M} \sum_{j>i} \frac{1}{2}\left(k_{i}+k_{j}\right) A_{i j}\right]^{2}}{\frac{1}{M} \sum_{j>i} \frac{1}{2}\left(k_{i}^{2}+k_{j}^{2}\right) A_{i j}-\left[\frac{1}{M} \sum_{j>i} \frac{1}{2}\left(k_{i}+k_{j}\right) A_{i j}\right]^{2}},
$$

where $M$ is the total number of edges in the network.

\subsubsection{Network models}

\subsubsection{The Erdős-Rényi model}

The random networks began to be studied in order to know the properties of the networks as a function of the number of random connections. Erdős and Rényi () were one of the first scholars to study random networks. The model begins with $N$ nodes and connects every pair of nodes with probability $p$, creating a graph with approximately $p N(N-1) / 2$ edges distributed randomly. The degree distribution converges to (NEWMAN, 2010a)

$$
P(k)=\frac{\langle k\rangle^{k} e^{-\langle k\rangle}}{k !} \text {. }
$$

The majority of nodes have approximately the same degree, It means that there is no presence of node with high degree, also called hubs. The distribution of the number of connections (degree) follows a Poisson distribution. Networks created by this process is called the $G_{N, p}$ ensemble. We will refer to this network as Erdôs Rényi (ER) networks. This model is used as a benchmark for many modelling and empirical studies. Networks generated by the ER model have a very large regularity.

\subsubsection{The Watts-Strogatz model}

Watts and Strogatz (1998) investigated the change in the network topology in terms of local clustering and minimum path length. They proposed a simple model that can be tuned through a regular and random network. We will refer to this network as Small World (SW) network. The procedure for generating the network starts from a ring-like lattice with $n$ nodes and $k$ edges per node. Then, each edge is rewired with probability $p$. This network structure or topology is highly clustered. They showed that the existence of even a few long-range connections greatly reduced the minimum path length of the network without affecting its local clustering to the same extent. It is known that the brain likely evolved to minimize the costs of information processing (BASSETT; BULLMORE, 2006). The small-world topology is associated with low wiring costs. To construct a SW network we can follow the following procedure: 
1. Place the nodes in a one-dimensional ring with periodic boundary conditions.

2. Connect every node to its respective $k$ nearest neighbours in both directions so that $k_{i}=2 k$.

3. Rewire each edge with probability $p$.

For small $p$, each short cut has a highly nonlinear effect on path length contracting the distance between the pair of vertices that it connects, but between their immediate neighbourhoods.

\subsubsection{Barabási-Albert model}

Until then, key features such as growing and preferential attachment, properties of real networks, had not been modelled by ER or SW network. Thinking of a network model that incorporates these two key features, Barabasi and Albert (1999) proposed a network model that provides a simple approach for obtaining power-law degree distribution of the form $P(k) \propto k^{-3}$. The model, which is based on preferential growth and attachment generates networks so that a small portion of the nodes has a substantially larger degree. Such nodes are called hubs. Many real-world networks such as the World Wide Web, movie actor collaboration, cellular network (BARABASI; ALBERT, 1999; FALOUTSOS; FALOUTSOS; FALOUTSOS, 1999) have a power-law degree distribution. We will refer to this network as Barabási-Albert (BA) network.

\subsubsection{Deactivation model}

(KLEMM; EGUÍLUZ, 2002) proposed a model of network growth that contains many properties observed in real-world networks such as power-law degree distribution and large clustering coefficient. The growth of the network is based on a vertex activation-deactivation dynamics. The model has the following characteristics i) power-law distribution for the degree, ii) preferential attachment, and negative correlation between age and attachment rate. The algorithm to construct the deactivation network model is given by the following steps:

1. A new node joins the network by attaching a link to each of the $m$ active nodes.

2. For each of the $m$ links of the new node it is decided randomly, with probability $\mu$, whether the link connects to the active node or connects to a random node.

3. The new node becomes active.

4. One of the active nodes is deactivated. The probability that node $i$ is chosen for deactivation is given by $p_{i}=a k_{i}^{-1}$, where $a=\sum_{j} k_{j}^{-1}$. The random nodes are chosen according to linear preferential attachment. The probability that node $j$ obtains a link is proportional to the node's degree $k$. 
In figure 2 we can see the degree distribution of deactivation model when $\mu=0$ recover the high clustering that is higher than in regular lattices, and $\mu=1.0$ is the BA model. For both parameters, the degree distribution follows a power law distribution.

Figure 2 - Degree distribution of deactivation model

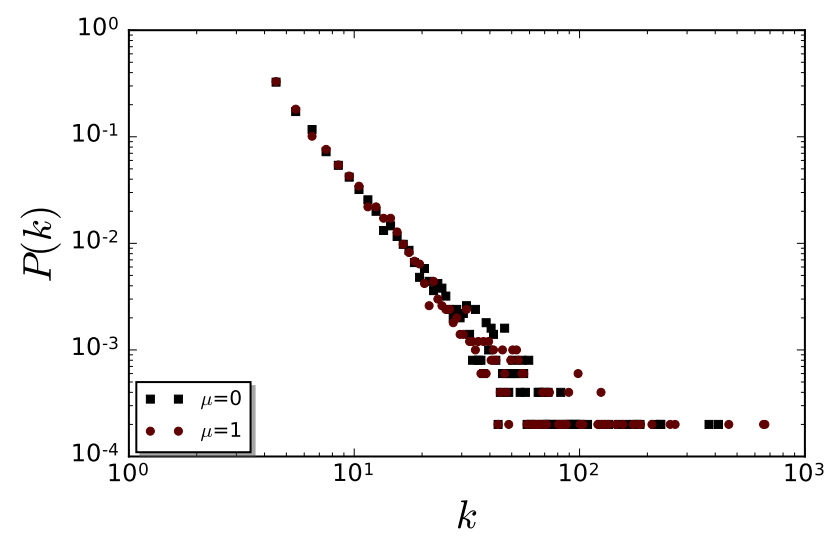

Source: Elaborated by the author.

In figure 3 shows the variation of the average shortest path length and the clustering coefficient with the parameter $\mu$.

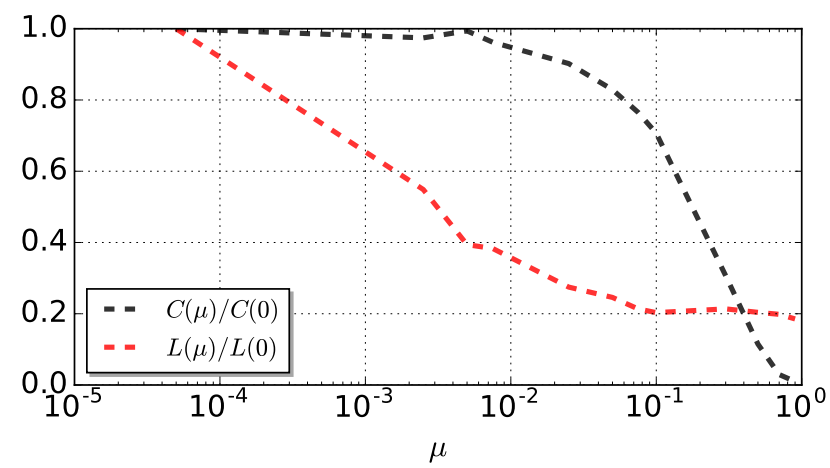

Figure 3 - Figure adapted from (KLEMM; EGUÍLUZ, 2002)

\subsubsection{Assortativity networks}

Generally, the assortativity of a network is determined for the degree of the nodes in the network. The existence of a degree-degree correlation between nodes is an important property of networks. It has been reported that different correlations arise in different kinds of networks brain networks tend to be assortative (BULLMORE; SPORNS, 2012; MURAKAMI et al., 2017). By other hand technological and biological systems have disassortative structures.

The characteristic usually referred to as assortativity is when nodes with many connections tent to connect to other nodes with high degree. In a disassortative network, high-degree 
Figure 4 - BA network with the degree correlated modified by the approach. (a) BA assortative com $\mathrm{r}=$ 0.32 e (b) BA dissortative com $r=-0.47$.

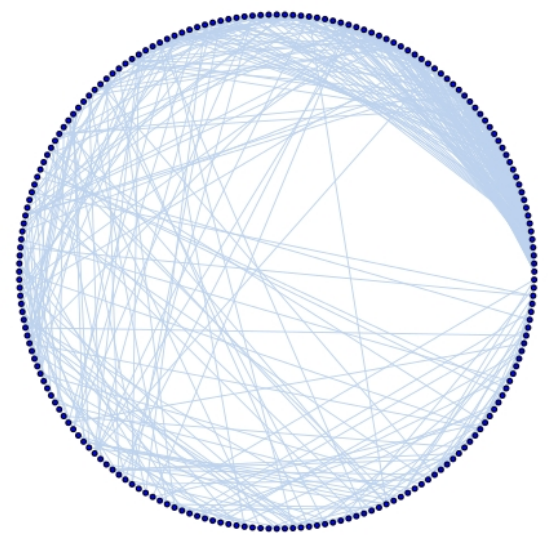

(a) Assortativity Network

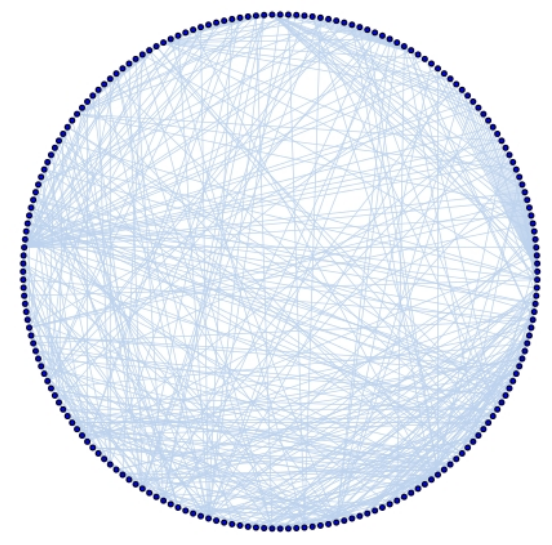

(b) Dissortativity Network

Source: Elaborated by the author.

nodes are less connected to one another. Assortativity provides information about the structure of a network, but also about dynamic behaviour of the network and robustness. In Figure 4 shown examples of assortative and disassortative networks.

In order to determine the exact influence of correlations many models have been proposed to build correlated networks (NEWMAN, 2003; XULVI-BRUNET; SOKOLOV, 2004). Here we consider the procedure in (ARRUDA et al., 2016), which is based on a simulated annealing procedure. This algorithm is based on two functions: (i) the perturbation function is a rewiring procedure that preserves the degree distribution of the network, but changes the large-scale degree-degree correlation and (ii) the energy function, which is minimized. The energy function is defined as $E_{t}=c\left(\rho_{t}+1\right)$, where $\rho_{t}$ is the assortativity of the network at time $t$, and $c$ is a parameter related to the desired degree-degree correlation.

Given an initial network configuration, an initial temperature, $T$ and a cooling factor $\beta$, the algorithm can be described by the following steps: (i) the energy function is initialized as $E_{0}$; (ii) while the number of iterations are less than a threshold or the optimal solution is not found the following steps are performed: (iii) a rewiring preserving the degree distribution is executed, according to the perturbation function; (iv) the new energy function, $E_{t+1}$, is calculated (v) $E_{t+1}-E_{t+1}<0$ then the new solution is accepted; (vi) the temperature is updated, $T=\beta T$; and (vii) increment the iteration counter. 


\subsection{Artificial neural network}

The first mechanism of an Artificial Neural Network (Artificial Neural Network (ANNs)) for character recognition was developed by Rosenblatt (ROSENBLATT, 1958) called "Perceptron" using concepts of the model of McCulloch and Pitts (1943). However, due to the limitation of not being able to classify correctly objects non-linearly separable, caused that the scientific community lost the interest in this field of research that lasted almost 20 years. Subsequently to this time, there was an explosive development in this field in the mid 80, with the network models proposed by Hopfield which consist of a nonlinear associative memory, or content-addressable memory, capable to retrieve a pattern stored in memory in response to the presentation of an incomplete or noisy version of that pattern, the Boltzmann machine that is a generalization of Hopfield model, multilayer perceptron(MLP) and Kohonen's self organizing networks.

Mathematically, a neural network is a kind of triple $(N, V, w)$ with two sets $N, V$, where $N$ is the set of neurons and $V$ is a set whose elements are connections between neurons. The function $w$ define the weights, where $w(i, j)$ is the weight of connection between neuron $i$ and $j$. A neural network is not just a complex system, but a complex adaptive system, whose adaptation is obtained through the adjusting of weights according to rules inspired by real biological networks.

There are three essential paradigms of the learning process of a neural network: (i) unsupervised learning is the biologically most plausible method, once the input patterns are given; the network tries to identify similar patterns and to classify them into similar categories, (ii) reinforcement learning the network receives a logical or a real value after completion of a sequence, which defines whether the result is right or wrong and (iii) supervised learning, where the network weights can be changed according to the difference between the output of the network and the correct solution.

\subsubsection{Self-organization map}

Teuvo Kohonen (KOHONEN, 1982) proposed a theoretical study and computational simulations of an artificial neural network called self-organization map (Self-Organization map (SOM)), with two particular characteristics, the creation of a spatially organized map and the competitive learning of biological plausibility. Since its introduction, several variations of this network have been proposed and investigated such as growing SOM (SAKAR; MAMMONE, 1993; FRITZKE, 1993; ALAHAKOON; HALGAMUGE; SRINIVASAN, 2000), SOM with fast convergence (SU; CHANG, 2000), Fuzzy SOM (TSAO; BEZDEK; PAL, 1994; HU et al., 2004) and the growing hierarchical SOM (DITTENBACH; RAUBER; MERKL, 2002). There are many research papers published regarding the SOM used in various domains which include, image segmentation (ORTIZ et al., 2014; AGHAJARI; CHANDRASHEKHAR, 2017), text document clustering (KASKI et al., 1998; LIU; WU; LIU, 2011), speech analysis and recognition (CORRI- 
DONI; BIMBO; LANDI, 1996; MAJUMDER; BEHERA; SUBRAMANIAN, 2014) and many more.

The SOM combines an input layer with a competitive layer of processing neurons, which is organized as a two-dimensional regular grid of neurons or units, where each neuron $i$ is represented by a weights vector called model or codebook having the same dimensionality of the input patterns. It is able to convert nonlinear statistical relationships between high-dimensional data items into simple relationships on a low-dimensional map. Figure 5 provides the schematic diagram of this mapping. As we can see the whole network is fully connected, i.e. all input nodes are connected to all nodes in the output layer and each output node is weighted.

Figure 5 - Schematic Diagram of SOM

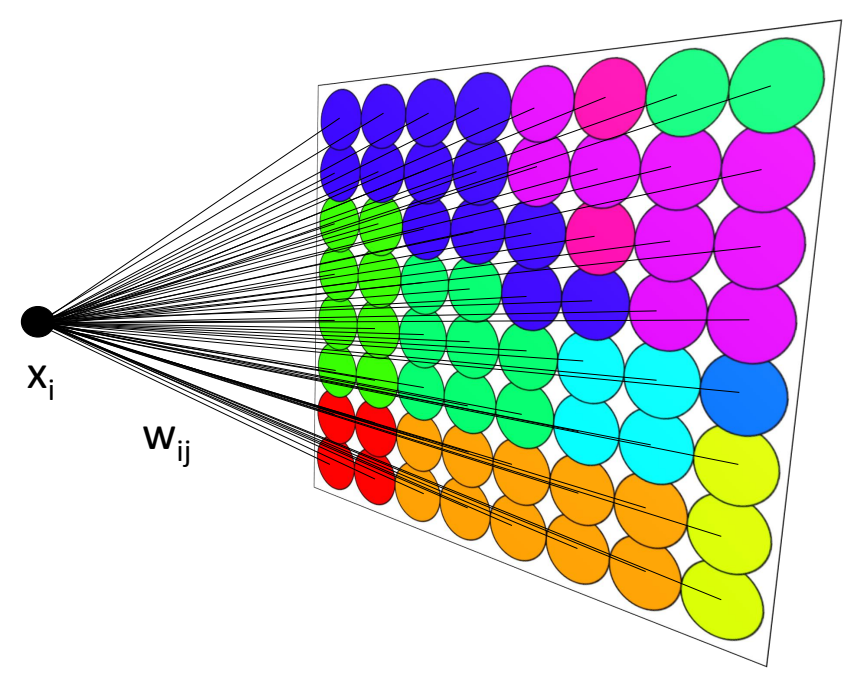

Source: Elaborated by the author.

SOM uses a competitive learning, in which the output neurons compete amongst themselves to be activated. The effect of the competitive learning is to move the stored pattern in the BMU neuron, a little bit closer to the input pattern. The Best Matching Unit (BMU) (Best Matching Unit) is the neuron of the output layer, that, in more similar to a given input pattern (KOHONEN, 2013). The competition between neurons has an important role in improving, sharpening, or even forming the features extracted from the data by each single neuron (ARBIB, 2003). The procedure is described as follows:

- After initialization, the SOM is trained for a number of epochs. A set of input patterns represented by vectors is presented to the codebook in a random order at each training cycle.

- Then, the neurons are competing with each other for the right to represent the input pattern, so that the neuron whose weight vector is more similar to the input pattern, according to a previously defined metric. The neuron who wins the competition is called BMU. 
- The BMU neuron is adapted, that is, the weights vector is updated in the sense of becoming more similar to the input pattern. Not only the BMU neuron is updated but all neighborhood neuron's weights are updated as well. Those updates depend on how far the adjusted neuron is located from the winner.

At each training cycle $t$ we calculate the euclidean distance of each weights vector $w_{i}(t)$ to the input pattern $x$.

$$
B M U=\operatorname{argmin}_{i}\left\{d\left(x(t), w_{i}(t)\right)\right\}
$$

where $x(t)$ is the input at iteration $t$, and $w_{i}(t)$ is the weight vector at iteration $t$. Neurons that are close to the BMU neuron are updated according to a gaussian neighbourhood function $h_{c i}$, that is expressed as

$$
h_{c i}(t)=\exp \left(\frac{-d_{c i}}{2 \sigma^{2}(t)}\right)
$$

where $d_{c i}$ denotes the smallest distance between a given $c t h$ neuron and the winning neuron, and $\sigma$ controls the scale of the neighborhood during training. The weights are then updated as

$$
w_{i}(t+1)=w_{i}(t)+\alpha(t) * h_{c i}(t) *\left(x(t)-w_{i}(t)\right)
$$

where $w_{i}(t+1)$ is the adjusted weight, $\alpha(t)$ is a learning rate function that decreases with time, $h_{c i}(t)$ represent a kernel neighborhood function over the lattice, defining which units and how much will they be affected by each input pattern. This update step has to be repeated for all the $w_{i}$ for every $x$ presented.

In practice the neurons are placed on a regular low dimensional grid and the kernel neighborhood function is defined as a monotonically decreasing function during learning. The parameter $\sigma$ represent the radius of neighborhood. A common choice to $\sigma(t)$ is the exponencial decay function as follow: $\sigma(t)=\sigma_{0} e^{-\frac{t}{\tau}}$ where $\sigma_{0}$ is the initial value of the radius of the neighbourhood and $\tau$ is a time parameter.

Through a process of learning the outputs become self-organised and the feature map between inputs and outputs is formed. The traditional SOM training algorithm, or called "online algorithm", assumes the updating of the synaptic weights of the neurons every time a data item is presented to the network. In figure 7 we can observe the map created by SOM network after a learning step. Was used the digits dataset( 0 to 9 ) to training the network.

Figure 6 - Digits dataset

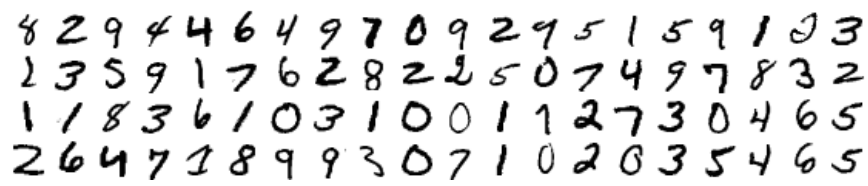

Source: Elaborated by the author. 


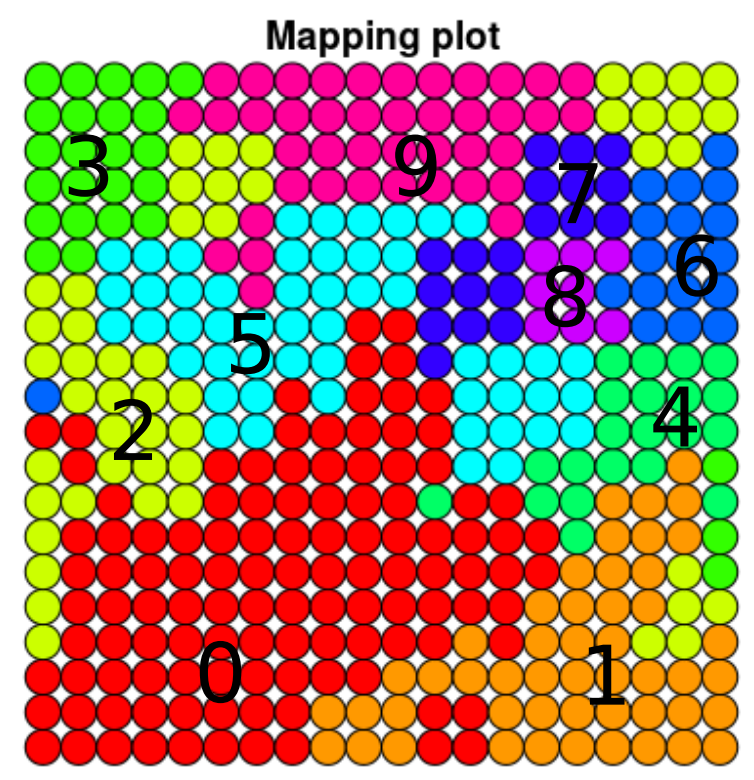

Source: Elaborated by the author.

\subsubsection{Variations in the parameters}

There are several ways to build algorithms derived from SOM, modifying the following aspects.

- The choice of the BMU neuron can be given not only considering the Euclidean distance, although it is the most used.

- The neighbourhood may have a regular, hexagonal or spherical shape. It can also be defined gradually, as the network grows, with the inclusion or removal of neurons during the training process.

\subsection{Data clustering}

There are several definitions of clustering of data in the literature. A first definition indicates that clustering is a method for exploratory analysis of data used to aid in the resolution of classification problems (BACKER, 1995). A second definition describes clustering as a technique for finding groups in the data in which objects belonging to a particular group share some characteristic or property relevant to the problem being studied (JAIN; DUBES, 1988). As well, there are many approaches proposed in the literature that have been explored and developed 
to perform clustering (GUHA; RASTOGI; SHIM", 2001; ZHANG; CHEN, 2003; WHITE; SMYTH, 2005; HUANG et al., 2013). Also, many of the commonly employed methods are defined in terms of similar assumptions about the data or consider analogous mathematical concepts (e.g, similarity matrices for spectral or graph clustering).

The purpose of clustering algorithms is to identify groups of objects, or clusters, that are more similar to each other than to other clusters. Such an approach to data analysis is closely related to the task of creating a model of the data, that is, defining a simplified set of properties that can provide an intuitive explanation about relevant aspects of a dataset. Clustering methods are generally more demanding than supervised approaches, but provide more insights about complex data.

For clustering of data there is a sequence of steps that must be considered. The stage of pre-processing of the data that implies the reduction of the number of attributes through the selection or extraction of characteristics. Next, to this step, similarity/dissimilarity measures between objects, which are referred to as measures of proximity, are used to decide whether to assign an object to a cluster or to join/divide clusters. A higher similarity value indicates a greater similarity between objects. Usually, a value of 1 indicates that two objects are identical. The dissimilarity measure works the opposite way. Clustering algorithms are applied to identify possible clusters structures in the data. Finally, the evaluation of the quality of the generated partitions is one of the most important issues in cluster analysis (HALKIDI; BATISTAKIS; VAZIRGIANNIS, 2001). A common approach for evaluation of clustering results is to use validity indexes. There are three approaches to verify the validity of clustering: i) external criteria: results are evaluated based on a predefined structure, which is imposed on a dataset and reflects an intuition about the data grouping structure; ii) internal criteria: this criterion can be evaluated from the proximity matrix, iii) relative criteria: the evaluation of a grouping structure is done comparing it with another grouping structure obtained by the same algorithm but with different parameters. Some measures are biased towards certain clusterings. For example NMI index gives higher value to the partition with a larger number of clusters. Therefore, the ideal is to consider more than one index to validate the output of each algorithm of clustering. Here, we adopt the most traditional indexes, which are the Jaccard Index (Jaccard Index (J)) (JACCARD, 1908), Adjusted Rand Index (Adjusted Rand Index (ARI)) (LAWRENCE; ARABIE, 1985), Fowlkes Mallows Index (Fowlkes Mallows Index (FM)) (FOWLKES, 1983) and Normalized Mutual Information (Normalized Mutual Information (NMI)) (STREHL; GHOSH; CARDIE, 2002).

In order to define the cluster quality metrics, we consider the following concepts. Let $U=\left\{u_{1}, u_{2} \ldots u_{R}\right\}$ represent the truth partition of a dataset which is usually generated by an expert in the data domain, where $u_{i}$ denote a subset of the objects associated with cluster $i$. Equivalently, let $V=\left\{v_{1}, v_{2} \ldots v_{C}\right\}$ represent the partition found by a cluster algorithm. We denote as $a$ the number of pairs of objects that are placed in the same group in both $U$ and $V$. Mathematically, $a$ 
can be computed by

$$
a=\sum_{i, j}\left(\begin{array}{c}
n_{i j} \\
2
\end{array}\right)
$$

where $n_{i j}$ is the number of objects belonging to both subset $u_{i}$ and $v_{j}$.

Let $b$ indicate the number of pairs of objects belonging to the same group in $U$ but different groups in $V$, i.e.

$$
b=\sum_{i}\left(\begin{array}{c}
n_{i} \\
2
\end{array}\right)-\sum_{i, j}\left(\begin{array}{c}
n_{i j} \\
2
\end{array}\right),
$$

where $n_{i .}=\sum_{j} n_{i j}$. Let $c$ be the number of pairs of objects belonging to different groups in $U$ and to the same group in $V$, which can be written as

$$
c=\sum_{j}\left(\begin{array}{c}
n_{\cdot j} \\
2
\end{array}\right)-\sum_{i, j}\left(\begin{array}{c}
n_{i j} \\
2
\end{array}\right),
$$

where $n_{. j}=\sum_{i} n_{i j}$

The Jaccard Index $(J)$, Adjusted Rand Index (ARI) and Fowlkes Mallows (FM) index can then be defined based on $a, b, c$ :

$$
\begin{gathered}
J=\frac{a}{a+b+c}, \\
\mathrm{ARI}=\frac{\sum_{i, j}\left(\begin{array}{c}
n_{i j} \\
2
\end{array}\right)-\left[\sum_{i}\left(\begin{array}{c}
n_{i} \\
2
\end{array}\right) \sum_{j}\left(\begin{array}{c}
n_{. j} \\
2
\end{array}\right)\right] /\left(\begin{array}{c}
n \\
2
\end{array}\right)}{1 / 2\left[\sum_{i}\left(\begin{array}{c}
n_{i} \\
2
\end{array}\right)+\sum_{j}\left(\begin{array}{c}
n_{. j} \\
2
\end{array}\right)\right]-\left[\sum_{i}\left(\begin{array}{c}
n_{i} \\
2
\end{array}\right) \sum_{j}\left(\begin{array}{c}
n_{. j} \\
2
\end{array}\right)\right] /\left(\begin{array}{c}
n \\
2
\end{array}\right)}, \\
\mathrm{FM}=a \frac{\sqrt{a+b} \sqrt{a+c}}{(a+b)(a+c)} .
\end{gathered}
$$

We also consider the normalized mutual information(NMI) as a quality metric because it quantifies the mutual dependence between two random variables based on well-established concepts of information theory (COVER; THOMAS, 2012). The NMI measure is defined as

$$
\operatorname{NMI}(C, T)=\frac{I(C, T)}{\sqrt{[H(C), H(T)]}} .
$$

where $C$ is the random variable denoting the cluster assignments of the points, and $T$ is the random variable denoting the underlying class labels on the points. $I(C, T)=H(C)-H(C \mid T)$ is the mutual information between the random variables $C$ and $T . H(C)$ is the Shannon entropy of C. $H(C \mid T)$ is the conditional entropy of $C$ given $T$.

Note that when the two sets of labels have a perfect one-to-one correspondence, the quality measures are all equal to unity.

\subsubsection{Taxonomies of clustering of data}

Several taxonomies have been proposed to organize the many different types of clustering algorithms into families (FRALEY; RAFTERY, 1998; JAIN et al., 2004). While some taxonomies 
categorize the algorithms based on their objective functions (JAIN et al., 2004), others aim at the specific structures desired for the obtained clusters. Kamber and Pei (HAN; PEI; KAMBER, 2011), classify clustering algorithms into five categories: (i) hierarchical methods, (ii) partitioning methods, (iii) density-based methods, (iv) grid-based methods, and (v) model-based methods.

\subsubsection{Hierarchical methods}

The hierarchical algorithms group creates a sequence of nested partitions. The graphical representation is a binary tree called the dendrogram. Two approaches can be considered in this type of clustering, that is, agglomerative and divisive. (1) In the agglomerative approach, start with $\mathrm{n}$ clusters with a single object. At each iteration, individual objects are added to existing clusters or join the two more similar groups until all elements are in only one group or by some stopping criteria. (2) (2) In the divisive approach, initially, all data is considered a unique group that is partitioned until each object is in a different group. To group two subsets of objects is used as linkage metric. These metrics are defined as follows: i) Single linkage: It is given by the distance between the objects of two clusters that are closest. It produces clusters of elongated form. It works well in well-separated clusters. ii) Complete linkage: describes the distance between the two most distant objects. Produces compact clusters, iii) Average linkage: merge group according to the mean of the distances between elements of distinct groups.

\subsubsection{Partitional methods}

In this type of approach the data are divided into $k$ groups, and between the groups, there is no hierarchy. For data partitioning, several heuristics have been proposed that help to consider an approximate solution in order to find the best partition (FACELI et al., 2011). The two most popular algorithms in this approach are the kmeans and the kmedoids. The kmeans requires input parameters the number of groups $(k)$ and a distance metric. Initially, each data point is associated with one of the $k$ clusters according to its distance to the centroids (clusters centres) of each cluster.

The final classification can strongly depend on the choice of the number of centroids (MACQUEEN et al., 1967). In addition, the k-means is not particularly recommended in cases where the clusters do not show convex distribution or have very different sizes (JAIN, 2010; STEINLEY, 2006). Moreover, the k-means algorithm is sensitive to the initial seed selection (JAIN; MURTY; FLYNN, 1999). Given these limitations, many modifications of this algorithm have been proposed (DUNN, 1973; HUANG, 1998). The kmedoide proved to be more robust than the Kmeans when there is the presence of noise or outliers. However, this method is not ideal for working with high-dimensional data because as the number of data increases, its efficiency decreases (FACELI et al., 2011). Another interesting example of partitional clustering algorithms is the clustering for large applications (clara)(KAUFMAN; ROUSSEEUW, 1990). This method takes into account multiple fixed samples of the dataset to minimize sampling bias and, subsequently, select the 
best medoids among the chosen samples, where a medoid is defined as the object $\mathrm{i}$ for which the average dissimilarity to all other objects in its cluster is minimal. This method is efficient for large amounts of data because it does not explore the whole neighbourhood of the data points (HAN; KAMBER, 2006), although the quality of the results has been found to strongly depend on the number of objects in the sample data.

\subsubsection{Methods based on density}

This type of algorithm consider the groups as dense regions of objects in a 1-dimensional space. Basically consists of growing a certain group as long as there are objects in the group with density in their neighbourhood, greater than a threshold. The Ordering Points To Identify the Clustering Structure (optics) (ANKERST et al., 1999a) and the Density-based spatial clustering of applications with noise (DBSCAN) (ESTER et al., 1996) are the best known algorithms in this approach. The optics is defined as a set of density-connected objects which is maximal densityreachability. The algorithm starting with a point and expands its neighbourhood like DBSCAN, but it explores the new point in the order of lowest to highest core-distance. The core-distance of an object $p$ is the smallest distance $\varepsilon$ between $p$ and an object in its $\varepsilon$ - a neighbourhood that $p$ would be a core object with respect to $\varepsilon$. Noise objects are the set of objects not contained in any cluster. optics has the ability to detect clusters with large density variations and irregularly shaped clusters. The difference between DBSCAN lies in that different densities are constructed simultaneously. Can be extracted any density-based clustering from this order with respect to MinPts and a clustering distance $\varepsilon_{\text {, }}$ and assigning cluster-memberships (ANKERST et al., 1999b).

\subsubsection{Methods based on models}

Model-based methods can be regarded as a general framework for estimating the maximum likelihood of the parameters of an underlying distribution to a given dataset. A well-known instance of model-based methods is the expectation-maximization algorithm (EM). Most commonly, one considers that the data from each class can be modelled by multivariate normal distributions, and, therefore, the distribution observed for the whole data can be seen as a mixture of such normal distributions. A maximum likelihood approach is then applied for finding the most probable parameters of the normal distributions of each class. The EM approach for clustering is particularly suitable when the dataset is incomplete (REDNER; WALKER, 1984; DEMPSTER; LAIRD; RUBIN, 1977). On the other hand, the clusters obtained from the method may strongly depend on the initial conditions (AGGARWAL; REDDY, 2013). In addition, the algorithm may fail to find very small clusters (FRALEY; RAFTERY, 1998; FRALEY; RAFTERY, 2002).

Another class of methods considered in our analyses is spectral clustering. These methods emerged as an alternative to traditional clustering approaches that were not able to define nonlinear discriminative hypersurfaces (NASCIMENTO; CARVALHO, 2011). The main advantage 
of spectral methods lies on the definition of an adjacency structure from the original dataset, which avoids imposing a prefixed shape for the clusters (FILIPPONE et al., 2008). The first step of the method is to construct an affinity matrix $A \in \mathbb{R}^{N x N}$, where the value in the $j$-th row and $k$-th column indicates the similarity between points $j$ and $k$. This matrix can be regarded as a weighted graph representation of the data. Then, the eigenvalues and eigenvectors of the matrix are used for partitioning the data according to a given criterion. Many different types of similarity matrices can be used, a popular choice being the Laplacian matrix (LUXBURG, 2007). One disadvantage of spectral methods is the costly process of calculating the eigenvectors of the similarity matrix (AGGARWAL; REDDY, 2013).

\subsubsection{Subspace- based methods}

In recent years, the efficient handling of high dimensional data has become of paramount importance and, for this reason, this feature has been desired when choosing the most appropriate method for obtaining accurate partitions. To tackle high dimensional data, subspace clustering was proposed (PARSONS; HAQUE; LIU, 2004). This method works by considering the similarity between objects with respect to distinct subsets of the attributes (KRIEGEL; KRÖGER; ZIMEK, 2012). The motivation for doing so is that different subsets of the attributes might define distinct separations between the data. Therefore, the algorithm can identify clusters that exist in multiple, possibly overlapping, subspaces (PARSONS; HAQUE; LIU, 2004).

\subsubsection{Final remarks}

In this chapter, we presented the basic concepts related to complex networks, the SOM network, and some methods of data clustering.

Other algorithms of clustering can be found in references (JAIN; DUBES, 1988; DUDA; HART; STORK, 2012; BISHOP et al., 2006; GAN; MA; WU, 2007). We see that availability of such a large number of clustering algorithms existing in the literature can affect the quality and usefulness of finding groups in the data because often an inadequate technique can be chosen for the problem to be treated. 
CHAPTER

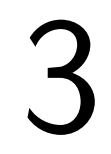

\section{ANALYSIS COMPARATIVE OF CLUSTERING ALGORITHM}

Most clustering algorithms involve several parameters, often operate in high dimensional spaces, and have to cope with noisy and incomplete data. Their performance can vary substantially for different applications and types of data. For these reason many clustering methods was proposed and several previous efforts for comparing clustering algorithms have been reported in the literature (JUNG; KANG; HEO, 2014; KINNUNEN et al., 2011; ABBAS et al., 2008; PIRIM et al., 2012; COSTA; CARVALHO; SOUTO, 2004; SOUTO et al., 2008; DOUGHERTY et al., 2002; BROHÉE; HELDEN, 2006; MAULIK; BANDYOPADHYAY, 2002; FRALEY; RAFTERY, 1998). But despite a large number of clustering methods have been proposed, there is no consensus on which methods are more suitable for a given dataset. As a consequence, it is important to comprehensively compare methods in many possible scenarios.

In order to account for the many possible variations of data, we quantified the sensitivity of the performance with respect to small changes in the data. We considered a variety of artificial datasets. The purpose of using artificial data is the possibility to obtain an unlimited number of samples and systematically change properties of a dataset such as the number of features, classes, number the objects per class and the correlation between features. Such features allow the clustering algorithms to be comprehensive and strictly evaluated in a vast number of circumstances. The method to generate different dataset create samples varying the number of class, the separation between classes and also can control both the variance and correlation distributions among the features for each class.

Subsequently, we performed a comparative analysis of SOM method with 8 well-known clustering methods. These methods have been implemented in several programming languages and packages. The current work focuses on the comparative analysis of several clustering algorithms found in popular packages available in the software R. Here, we chose the R programming language (R Development Core Team, 2006) for the analysis. This choice was motivated by 
the popularity of the $\mathrm{R}$ language in the data mining field, and by virtue of the well-established clustering packages it contains.

To evaluate the accuracy of each clustering algorithm, three methodologies were considered:

- We consider the default parameters of the algorithms provided by the R package. The reason for measuring performance using the default parameters is to consider the case where a researcher applies the classifier to a dataset without any parameter adjustment. This is a common scenario when the researcher is not a machine learning expert.

- We quantify the influence of the parameters of the algorithm on the accuracy. This is done by varying a single parameter of an algorithm while keeping the others at their default values.

- Finally, we analyze the performance by randomly varying all parameters of a clustering method. This procedure allows the quantification of certain properties such as the maximum accuracy attained and the sensibility of the algorithm to parameter variation.

The chapter is structured as follows. In section 3.1 we present the background and related works on this topic. In section 3.2 we present the $\mathrm{R}$ packages implementing the chosen clustering algorithms. In section 3.3 we detail the description of the method to generate the dataset that will be used in all the experiments. In section 3.5 we present the performance results obtained for the default parameters 3.5.1, for single parameter variation 3.5.2 and for random parameter sampling 3.5.3. Finally, the final considerations are in section 3.6.

\subsection{Background and related works}

Previous approaches for comparing the performance of clustering algorithms can be divided according to the nature of used datasets. While some studies use either real-world or artificial data, others employ both types of datasets to compare the performance of several clustering methods. Another common approach for comparing clustering algorithms considers using a mixture of real world and artificial data (e.g. (VERMA; MEILA, 2003; MAULIK; BANDYOPADHYAY, 2002; PIRIM et al., 2012; DOUGHERTY et al., 2002; BROHÉE; HELDEN, 2006)). In (MAULIK; BANDYOPADHYAY, 2002), the performance of k-means, single linkage and simulated annealing (SA) was evaluated, considering different partitions obtained by validation indexes. The authors used two real-world datasets obtained from UCI machine learning respository and three artificial datasets (having two dimensions and 10 clusters). They proposed a new validation index called $I$ index that measures the separation based on the maximum distance between clusters and compactness based on the sum of distances between objects and their respective centroids. They found that such an index was the most reliable among other 
considered indices, reaching its maximum value when the number of clusters is properly chosen. In addition, the authors concluded that the studied SA-based algorithms outperformed the traditional k-means. A systematic quantitative evaluation of four graph-based clustering methods was performed in (BROHÉE; HELDEN, 2006). The compared methods were: Markov clustering (MCL), restricted neighbourhood search clustering (RNSC), superparamagnetic clustering (SPC), and molecular complex detection (MCODE). Six datasets modelling protein interactions in the Saccharomyces cerevisiae and 84 random graphs were used for the comparison. For each algorithm, the robustness of the methods was measured in a twofold fashion: the variation of performance was quantified in terms of changes in the (i) methods parameters and (ii) dataset properties. In the latter, connections were included and removed to reflect uncertainties in the relationship between proteins. The restricted neighbourhood search clustering method turned out to be remarkably robust to variations in the choice of method parameters, whereas the other algorithms were found to be more robust to dataset alterations.

A comparative analysis using real world dataset is presented in several works (KOU; PENG; WANG, 2014; COSTA; CARVALHO; SOUTO, 2004; ERMAN; ARLITT; MAHANTI, 2006; SOUTO et al., 2008; KINNUNEN et al., 2011; JUNG; KANG; HEO, 2014). Some of these works are reviewed briefly in the following. In (KOU; PENG; WANG, 2014), the authors propose an evaluation approach based on a multiple criteria decision making in the domain of financial risk analysis over three real-world credit risk and bankruptcy risk datasets. More specifically, clustering algorithms are evaluated in terms of a combination of clustering measurements, which includes a collection of external and internal validity indexes. Their results show that no algorithm can achieve the best performance on all measurements for any dataset and, for this reason, it is mandatory to use more than one performance measure to evaluate clustering algorithms.

In (KINNUNEN et al., 2011), a comparative analysis of clustering methods was performed in the context of text-independent speaker verification task, using three datasets of documents. Two approaches were considered: clustering algorithms focused on minimizing a distance based objective function and a Gaussian model-based approach. The following algorithms were compared: k-means, random swap, expectation-maximization, hierarchical clustering, selforganized maps (SOM) and fuzzy c-means. The authors found that the most important factor for the success of the algorithms is the model order, which represents the number of the centroid or Gaussian components (for Gaussian models-based approaches) considered. Overall, the recognition accuracy was similar for clustering algorithms focused on minimizing a distance based objective function. When the number of clusters was small, SOM and hierarchical methods provided significantly poorer accuracy than the other methods. Finally, a comparison of the computational efficiency of the methods revealed that the hierarchical method is the fastest clustering algorithm in the considered dataset.

In (SOUTO et al., 2008), five clustering methods were studied: k-means, multivariate 
Gaussian mixture, hierarchical clustering, spectral and nearest neighbour methods. Four proximity measures were used in the experiments: Pearson and Spearman correlation coefficient, cosine similarity and the Euclidean distance. The algorithms were evaluated in the context of 35 gene expression data from either Affymetrix or cDNA chip platforms, using the ARI index for performance evaluation. The multivariate Gaussian mixture method provided the best performance in recovering the actual number of clusters of the datasets. The k-means method displayed similar performance. In this same analysis, the hierarchical method led to limited performance, while the spectral method showed to be quite sensitive to the proximity measure employed.

In (COSTA; CARVALHO; SOUTO, 2004), experiments were performed to compare five different types of clustering algorithms: Cluster identification via connectivity kernels (CLICK), self-organized mapping-based method (SOM), k-means, hierarchical and dynamical clustering. Data sets of gene expression time series of the Saccharomyces cerevisiae yeast were used. A $\mathrm{k}$-fold cross-validation procedure was considered to compare different algorithms. The authors found that k-means, dynamical clustering and SOM obtained high accuracy in all experiments. On the other hand, hierarchical clustering presented a poor performance in clustering larger datasets, yielding low accuracy in some experiments.

A comparative analysis using artificial data is presented in (MINGOTI; LIMA, 2006; MANGIAMELI; CHEN; WEST, 1996; PARSONS et al., 2004). In (PARSONS et al., 2004), two subspace clustering methods were compared: MAFIA (Adaptive Grids for Clustering Massive Data Sets) (BURDICK; CALIMLIM; GEHRKE, 2001) and FINDIT (A fast and intelligent subspace clustering algorithm using dimension voting) (PARSONS; HAQUE; LIU, 2004). The artificial data, modelled according to a normal distribution, allowed the control of the number of dimensions and instances. The methods were evaluated in terms of both scalability and accuracy. In the former, the running time of both algorithms was compared for a different number of instances and features. In addition, the authors assessed the ability of the methods in finding adequate subspaces for each cluster. They found that MAFIA discovered all relevant clusters, but one significant dimension was left out in most cases. Conversely, the FINDIT method performed better in the task of identifying the most relevant dimensions. Both algorithms were found to scale linearly with the number of instances, however, MAFIA outperformed FINDIT in most of the tests.

\subsection{Package in $\mathbf{R}$}

The clustering methods considered in our analysis were described in section 2.3 and the respective libraries and functions in $\mathrm{R}$ employing are listed below.

The k-means whose routine in the $\mathrm{R}$ language is the k-means function from the stat package, which contains the implementation of MacQueen et al. (1967) and Hartigan and Wong 
(1979). The Clara algorithm employed here is available as the clara function contained in the cluster package Kaufman and Rousseeuw (1990). For hierarchical approach, it was considered the agnes routine from the cluster package. Four well-known linkage criteria are available in agnes, namely single linkage, complete linkage, Ward's method, and weighted average linkage.

The package mclust (FRALEY; RAFTERY, 1999) provides iterative EM (ExpectationMaximization) methods for maximum likelihood estimation in parameterized Gaussian mixture models. Functions estep and mstep implement the individual steps of the EM iteration. A related algorithm that is also used is the hcmodel, which can be found in the $h c$ function of the mclust package.

The function specc from the kernlab R package (KARATZOGLOU et al., 2004) implement an spectral algorithm. Lastly the $h d d c$ function from package HDclassif (BERGÉ; BOUVEYRON; GIRARD, 2012) implements a subspace clustering method. The R routine used for SOM algorithm was the function som from package som. The R package implements the standard SOM (YANG, 2004).

\subsection{Method description}

The proper comparison of clustering algorithms requires a robust artificial data generation method to produce a variety of datasets. For such a task, we apply a methodology based on a previous work by Hirschberger et al. (HIRSCHBERGER; QI; STEUER, 2007). The procedure can be used to generate samples characterized by $F$ features and separated into $C$ classes. In addition, the method can control both the variance and correlation distributions among the features for each class. The artificial dataset can also be generated by varying the number of objects per class, $N_{e}$, and the expected separation, $\alpha$, between the classes.

The main difficulty in generating datasets with the aforementioned properties is the definition of a proper covariance matrix $R$ for the considered features. A valid covariance matrix must be positive semi-definite (HORN; JOHNSON, 2012), which is hard to ensure. However, for a given matrix $\mathbf{G} \in \mathbb{R}^{n \times m}$, the matrix $R=\mathbf{G G}^{T}$ is guaranteed to be positive semi-definite (HORN; JOHNSON, 2012). Thus any random matrix $\mathbf{G}$ can define a valid respective covariance matrix. As a consequence, additional constraints on matrix $\mathbf{G}$ can be imposed for the generation of datasets with the required properties. Hirschberger et al. (HIRSCHBERGER; QI; STEUER, 2007) developed a robust approach to generate such a matrix given the first two statistical moments of the co-variance distribution of a set of $F$ artificial features. The resulting covariance matrix contains variances and co-variances drawn from such distribution. Here we consider a normal distribution to represent the elements of $\mathbf{R}$. For each class $i$ in the dataset, a covariance matrix $\mathbf{R}_{i}$ of size $F \times F$ is generated, and this matrix is used to define $N_{e}$ objects for the classes. This means that pairs of features can have distinct correlation for each generated class. Then, the generated class values are divided by $\alpha$ and translated by $s_{i}$, where $s_{i}$ is a random 
(a)

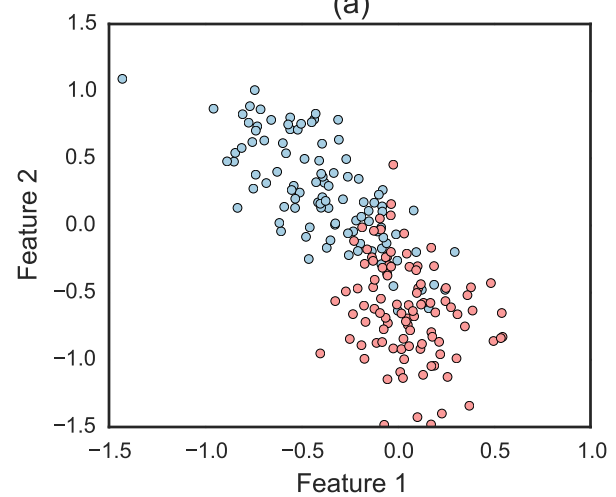

(c)

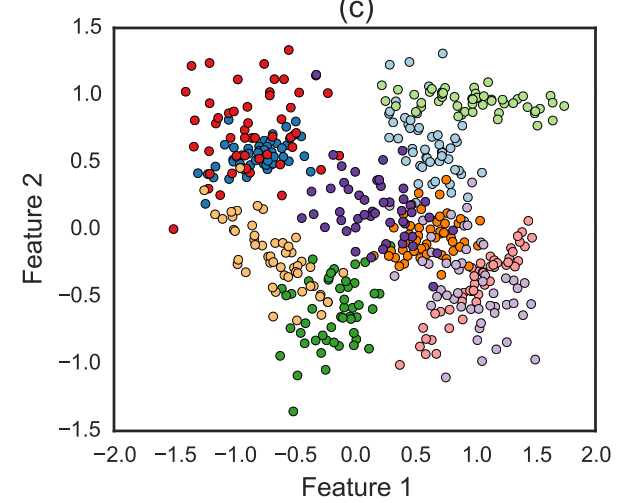

(b)

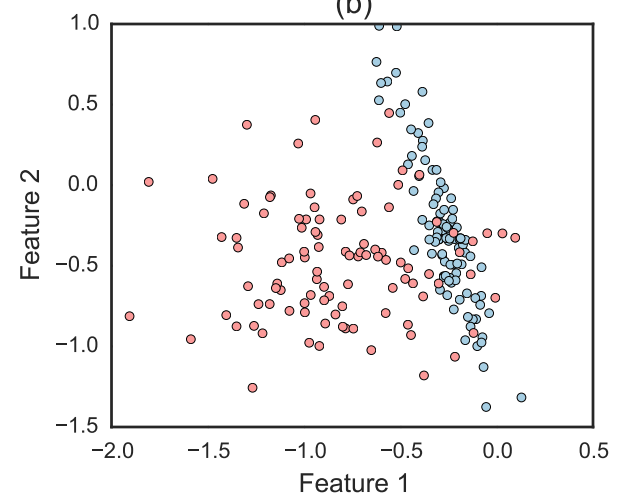

(d)

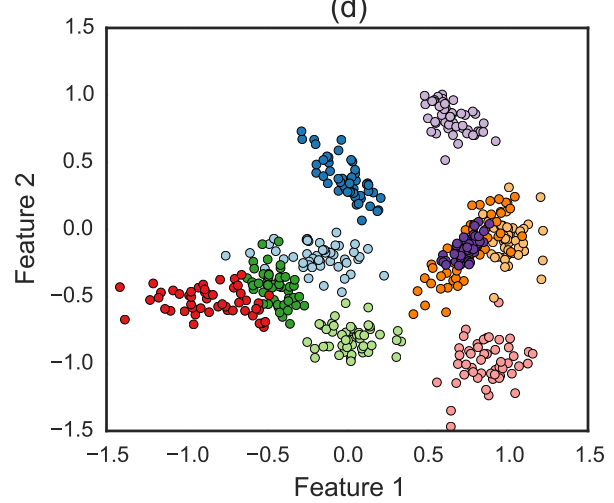

(a) Examples of artificial datasets generated by the methodology. The parameters used for each case are (a) $\mathrm{C}=2, N_{e}=100$ and $\alpha=3.3$. (b) $\mathrm{C}=2, N_{e}=100$ and $\alpha=2.3$. (c) $\mathrm{C}=10, N_{e}=50$ and $\alpha=4.3$. (d) $\mathrm{C}=10, N_{e}=50$ and $\alpha=6.3$. Note that each class can present highly distinct properties due to differences in correlation between their features.

Source: Elaborated by the author.

variable described by a uniform random distribution defined in the interval $[-1,1]$. Parameter $\alpha$ is associated with the expected distances between classes. Such distances can have different impacts on the clustering results depending on the number of objects and features used in the dataset. Notice that such a procedure for the generation of artificial datasets was previously used in (AMANCIO et al., 2014). The procedure can be used to generate samples characterized by $F$ features and separated into $C$ classes. In addition, the method can control both the variance and correlation distributions among the features for each class. The artificial dataset can also be generated by varying the number of objects per class, $N_{e}$, and the expected separation, $\alpha$, between the classes.

In Figure 8a, we show some examples of artificially generated data. For visualization purposes, all considered cases contain $F=2$ features. The parameters used for each case are described in the caption of the figure. Note that the methodology can generate a variety of dataset configurations, including variations in features correlation for each class.

In this study, we considered the following values for the artificial dataset parameters: 
- Number of classes $(C)$ : The generated datasets are divided into $C=\{2,10,50\}$ classes.

- Number of features $(F)$ : The number of features to characterize the objects is $F=$ $\{2,10,50\}$.

- Number of object per class $\left(N_{e}\right)$ : we considered $N e=\{5,50,100\}$ objects per class. In our experiments, in a given generated dataset, the number of instances for each class is constant.

- Mixing parameter $(\alpha)$ : This parameter has a non-trivial dependence on the number of classes and features. Therefore, for each dataset, the value of this parameter was tuned so that no algorithm would achieve an accuracy of $0 \%$ or $100 \%$.

We refer to datasets containing 2, 10 and 50 features as DB2F, DB10F, DB50F respectively. Such datasets are composed of all considered number of classes, $C=\{2,10,50\}$, and 50 elements for each class (i.e., $\mathrm{Ne}=50$ ). In some cases, we also indicate the number of classes considered for the dataset. For example, dataset DB2C10F contains 2 classes, 10 features and 50 elements per class. For each case, we consider 10 realizations of the dataset. Therefore, 360 datasets were generated in total. The accuracy of each considered clustering algorithm was evaluated using three methodologies. In the first methodology, we consider the default parameters of the algorithms provided by the $\mathrm{R}$ package. The reason for measuring performance using the default parameters is to consider the case where a researcher applies the classifier to a dataset without any parameter adjustment. This is a common scenario when the researcher is not a machine learning expert. In the second methodology, we quantify the influence of the parameters of the algorithm on the accuracy. This is done by varying a single parameter of an algorithm while keeping the others at their default values. The third methodology consists in analyzing the performance by randomly varying all parameters of a classifier. This procedure allows the quantification of certain properties such as the maximum accuracy attained and the sensibility of the algorithm to parameter variation.

\subsection{Description of the clustering algorithms' parameters}

In the following, we provide a brief description about the parameters of the clustering algorithms considered in the experiments. We note that, since some algorithms do not have a default value for the number of clusters, in all cases we set this parameter as the number of clusters in the dataset.

\subsection{1 k-means clustering}

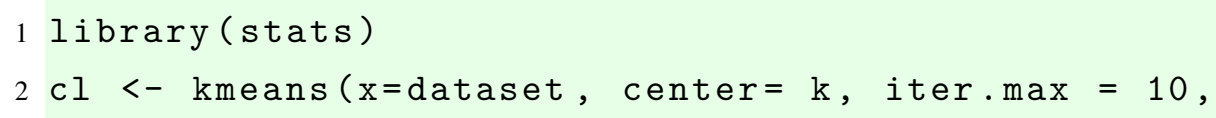


The k-means algorithm used in the main text has the following parameters:

- iter.max: integer, the maximum number of iterations. Default value: 10.

- nstart: integer, indicates how many random sets should be chosen. Default value: 1.

- algorithm: string, implementation of the k-means algorithm to use. Default value: "HartiganWong".

- center: integer, number of clusters. Default value: number of clusters in dataset.

\subsubsection{Clustering for large applications (clara)}

1 library (cluster)

2 cl <- $\operatorname{clara}(\mathrm{x}=$ dataset, $\mathrm{k}=\mathrm{k}$, metric = 'euclidean', samples = 5, $\operatorname{sampsize}=\min (\operatorname{nrow}($ dataset $), 40+2 * \mathrm{k}), \operatorname{rngR}=$ FALSE) \$cluster 3

The algorithm has the following parameters:

- metric: string, specifies the metric to be used for calculating dissimilarities between observations. Default value: "euclidean".

- sample: integer, number of samples to be drawn from the dataset. Default value: 5 .

- sampsize: integer, number of observations in each sample. sampsize should be larger than the number of clusters. Default value: $\min (N, 40+2 k)$, where $N$ is the number of objects.

- rngR: boolean, whether R's random number generator should be used instead of the primitive clara. Default value: false.

- $\mathrm{k}$ : integer, the number of clusters. Default value: number of clusters in dataset.

\subsubsection{Hierarchical clustering}

1 library (cluster)

2 cl <- cutree (agnes (dataset, metric = 'euclidean', stand = FALSE, method = 'average'), $\mathrm{k}=\mathrm{k}$ ) ;

The hierarchical method has the following options:

- metric: string, metric to use for calculating distances between samples. Default value: "Euclidean". 
- method: string, clustering method to use. Default value: "average".

- par.method: integer, specifies the parameter for the dissimilarity calculation in some methods. Default value: 0 .

- $\mathrm{k}$ : integer, the number of clusters. Default value: number of clusters in dataset.

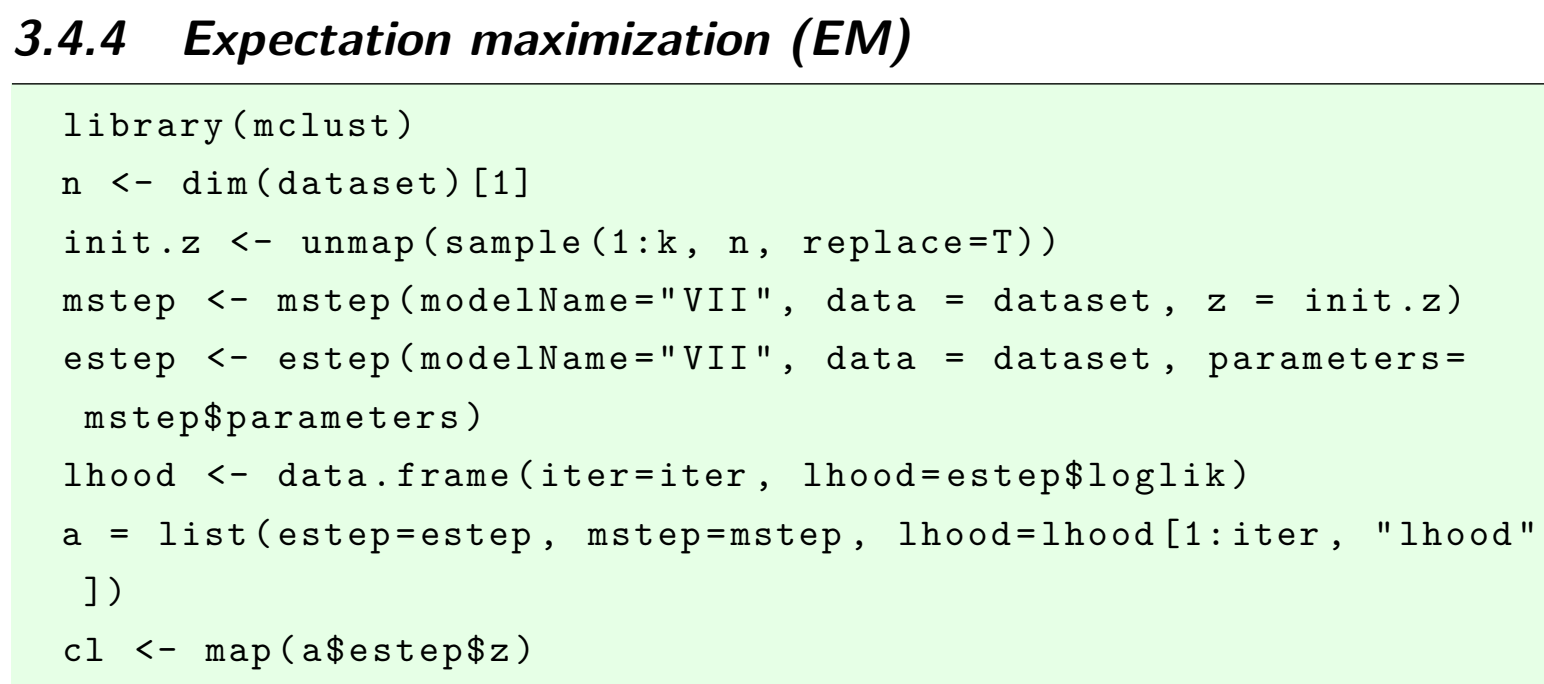

The algorithm used for expectation maximization clusterization is provided by the mclust package. Two routines of the package are used for applying the method:

- mstep: Maximization step in the EM algorithm for parametric Gaussian mixture models.

- z: string, conditional probability of the $i$-th observation belonging to the $k$-th component of the mixture. Default value: "random".

- modelName: string, indicates the model to be used. Default value: "VII".

- estep: Implements the expectation step of EM algorithm for parameterized Gaussian mixture models.

- modelName: string, indicates the model to be used. Default value: "VII".

- parameters: List containing the mean, variance and mixing proportion for each component. These parameters are usually obtained in the expectation mstep.

\subsection{5 hcmodel clustering}

1 library (mclust);

2 cl <- hclass (hc(as matrix (dataset), modelName = 'VVV', use = ' VARS'), k)

Provided by the mclust package. The hc routine employing the hcmodel has the following parameters: 
- modelName: string, indicates the model to be used. Default value: "VVV".

- use: string, specify what type of data/transformation should be used for model-based hierarchical clustering. Default value: "VARS".

- k: integer, number of clusters. Default value: number of clusters in dataset.

\subsubsection{Spectral algorithm}

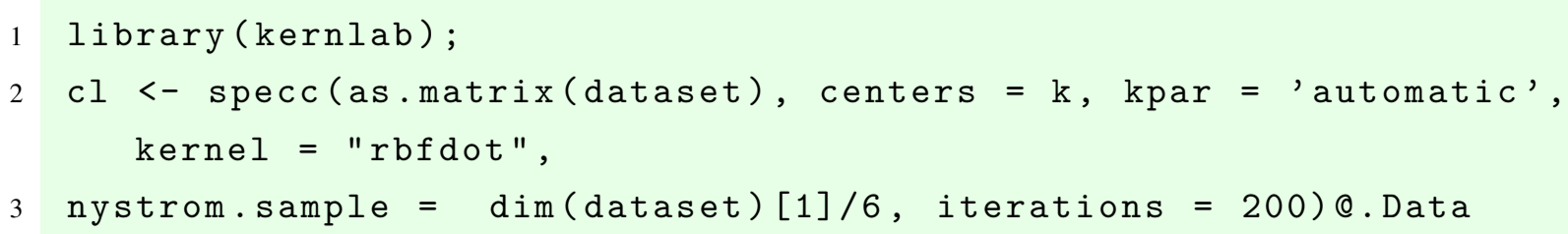

The routine specc of the kernlab package has the following options:

- centers: integer, number of clusters. Default value: number of clusters in dataset.

- kernel: string, the kernel function used in computing the affinity matrix. Default value: "rbfdot". The following options are available:

- rbfdot: Radial Basis kernel function ("Gaussian").

- polydot: Polynomial kernel function.

- vanilladot: Linear kernel function.

- tanhdot Hyperbolic tangent kernel function.

- laplacedot Laplacian kernel function.

- besseldot: Bessel kernel function.

- anovadot: ANOVA RBF kernel function.

- splinedot: Spline kernel.

- stringdot: String kernel

- kpar: string, the kernel parameter can also be set to a user defined function of class "kernel" by passing the function name as an argument. Default value: "automatic".

- nystrom.sample: float, proportion of data to use when estimating sigma. Default value: $N_{b} / 6$, where $N_{b}$ is the number of objects.

- iterations: integer, the maximum number of iterations. Default value: 200. 


\subsubsection{Subspace algorithm}

cl <- hddc(dataset, $K=1: k$, model=c(1), algo='EM', init='kmeans', mini.nb=c $(5,10)$, min. individuals $\left.\left.=2, d_{-} \max =100\right) \$ c l a s s\right)$

The routine used for subspace clustering, contained in the HDclassif package, is called hddc (High Dimensional Data Clustering). It has the following parameters:

- model: integer or string, 14 models can be used: 12 models with class specific orientation matrix and two models with common covariance matrix. Default value: "akjbkQkdk" or 1.

- $\mathrm{k}$ : designates the number of clusters. The algorithm selects the result with the maximum BIC value. Default value: the selected $\mathrm{k}$ is in the default interval $(1,10]$.

- algo: string, the algorithm used for clustering. Can be either EM, CEM (Classification EM) or SEM (Stochastic EM). Default value: "EM".

- init: string, how to the initial class assignments are done. Default value: "kmeans". Four initializations have been implemented:

- random: each observation is randomly assigned to a class.

- kmeans: the initial class of each observation is provided by the k-means algorithm.

- param: initializes according to a multivariate normal distribution.

- mini-em: the EM algorithm is run $m$ times for $n b$ iterations, the result with the highest likelihood is kept as the initialization of the algorithm.

- mini.nb: integer, used when parameter init is "mini-em". It is an array of length 2 containing $m$ and $n b$. Default value: $(5,10)$.

- min.individuals, integer, is used to control for the minimum population of a class. The value of 'min.individuals' cannot be lower than 2

\subsection{Results and Discussion}

\subsubsection{Performance when using default parameters}

In this experiment, we evaluated the performance of the classifiers for all datasets described in Section 3.3. All unsupervised algorithms were set with their default configuration of parameters. For each algorithm, we divide the results according to the number of features contained in the dataset. In other words, for a given number of features, $F$, we used datasets with $C=\{2,10,50\}$ classes, and $N_{e}=\{5,50,100\}$ objects for each class. Thus, the performance results obtained for each $F$ corresponds to the performance averaged over a distinct number of 
classes and objects per class. We note that the algorithm based on subspaces cannot be applied to datasets containing 2 features, and therefore its accuracy was not quantified for such datasets.

In Figure 9, we show the obtained values for the four considered performance metrics. The results indicate that all performance metrics provide similar results. Also, the hierarchical method seems to be strongly affected by the number of features in the dataset. In fact, when using 50 the hierarchical method provided the worst results among all methods. The k-means, spectral benefit from an increment in the number of features. Interestingly, the homodel has a better performance in the datasets containing 10 features than in those containing 2 and 50, which suggests an optimum performance for this algorithm for datasets containing around 10 features. It is also clear that for 2 features the performance of the algorithms tends to be similar. On the other hand, a larger number of features induce marked differences in performance.

We use the Kruskal-Wallis test (MCKIGHT; NAJAB, 2010), a one-way ANOVA nonparametric test, to explore the statistical differences in performance when considering a distinct number of features in clustering methods. First, we test whether the difference in performance is significant for 2 features. For this case, the Kruskal-Wallis test returns a p-value of $p=0.00017$, with a chi-squared distance of $\chi^{2}=21.12$. Therefore, the difference in performance is statistically significant when considering all algorithms. For data sets containing 10 features, a p-value of $p=2.06 \times 10^{-7}$ is returned by the Kruskal-Wallis test, with a chi-squared distance of $\chi^{2}=44.08$ ). For 50 features, the test returns a $p$-value of $p=3.59 \times 10^{-7}$, with a chi-squared distance of $\chi^{2}=42.83$ ). Therefore, the null hypothesis of the Kruskal-Wallis test is rejected. This means that the algorithms indeed have significant differences in performance for 2, 10 and 50 features, as indicated in Figure 9.

In order to verify the influence of the number of objects used for classification, we also calculated the average accuracy for datasets separated according to the number of objects $N_{e}$. The result is shown in Figure 10. We observe that the impact that changing $N_{e}$ has on the accuracy depends on the algorithm. Surprisingly, the hierarchical, k-means and Clara methods attain lower accuracy when more data is used. The result indicates that these algorithms are less robust with respect to the larger overlap between the clusters due to an increase in the number of objects. We also observe that a larger $N_{e}$ enhances the performance of the hcmodel. This result is in agreement with (BERGÉ; BOUVEYRON; GIRARD, 2012).

The results obtained for the default parameters are summarized in Table 1. The table is divided into four parts, each part corresponds to a performance metric. For each performance metric, the value in the row $i$ and column $j$ of the table represents the average performance of the method in the row $i$ minus the average performance of the method in column $j$. The last column of the table indicates the average performance of each algorithm. We note that the averages were taken over all generated datasets.

The results shown in Table 1 indicate that the spectral algorithm tends to outperform other algorithms by at least $10 \%$. On the other hand, the hierarchical method attained a poor 

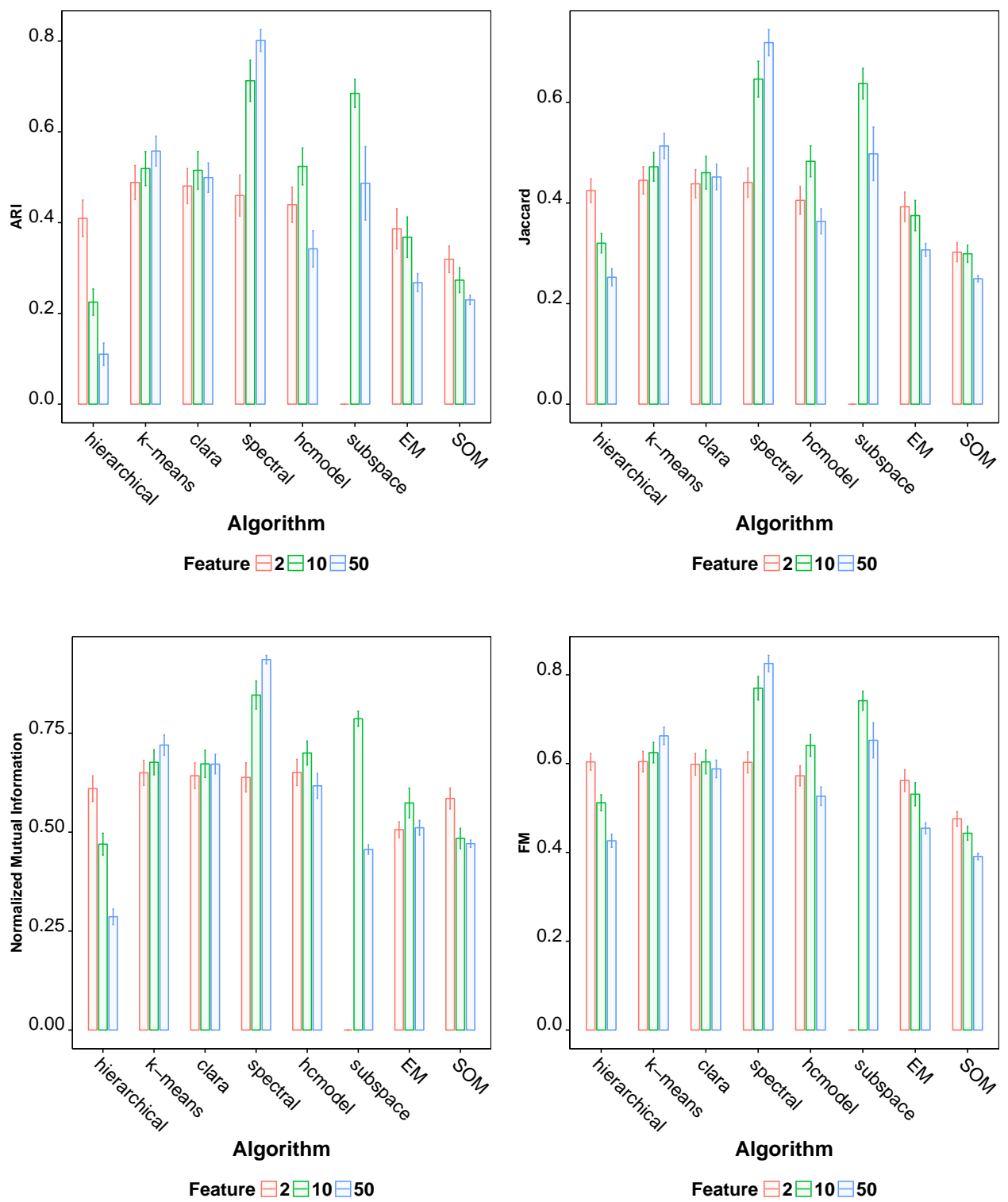

Figure 9 - Average performance of the seven considered clustering algorithms according to the number of features in the dataset. All datasets described in Section 3.3 were used for evaluation. The averages were calculated separately for datasets containing 2, 10 and 50 features. The considered performance indexes are (a) adjusted Rand, (b) Jaccard, (c) normalized mutual information and (d) Fowlkes Mallows.

performance in all considered cases. Another interesting result is that the k-means and Clara provide equivalent performance when considering all datasets. The SOM algorithm has a performance similar to the EM. In light of the results, we can conclude that the spectral method is to be preferred when no optimization of parameters values is performed. 


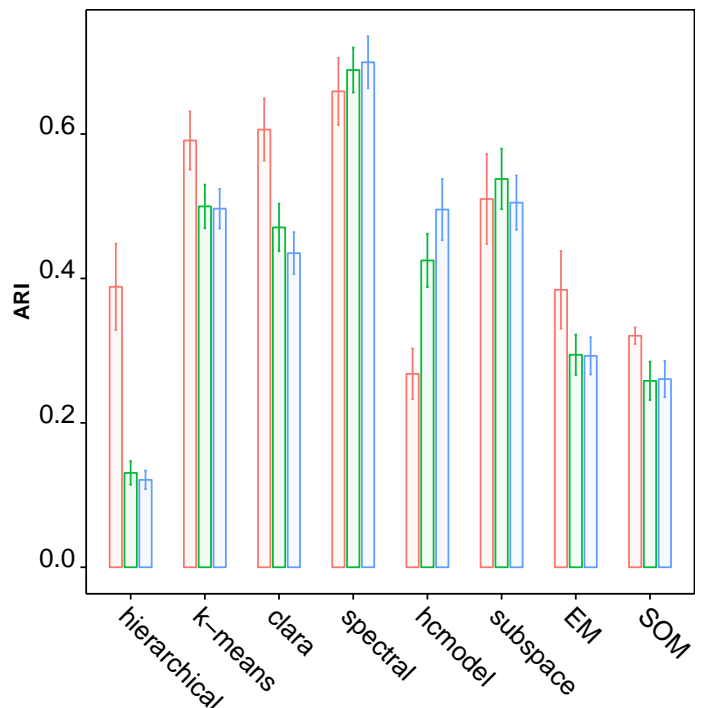

Algorithm

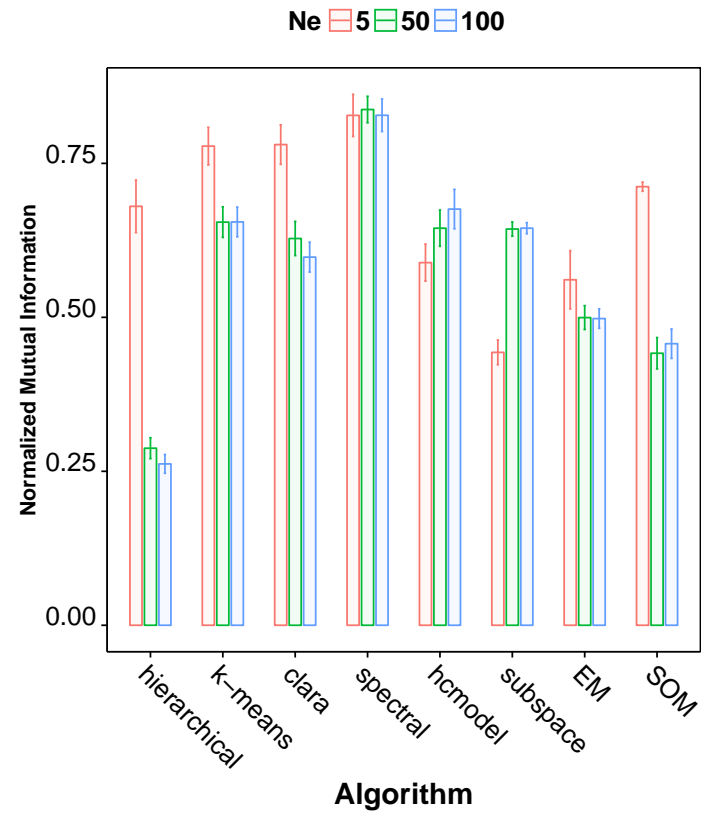

$\mathrm{Ne} \boxminus 5 \boxminus 50 \boxminus 100$

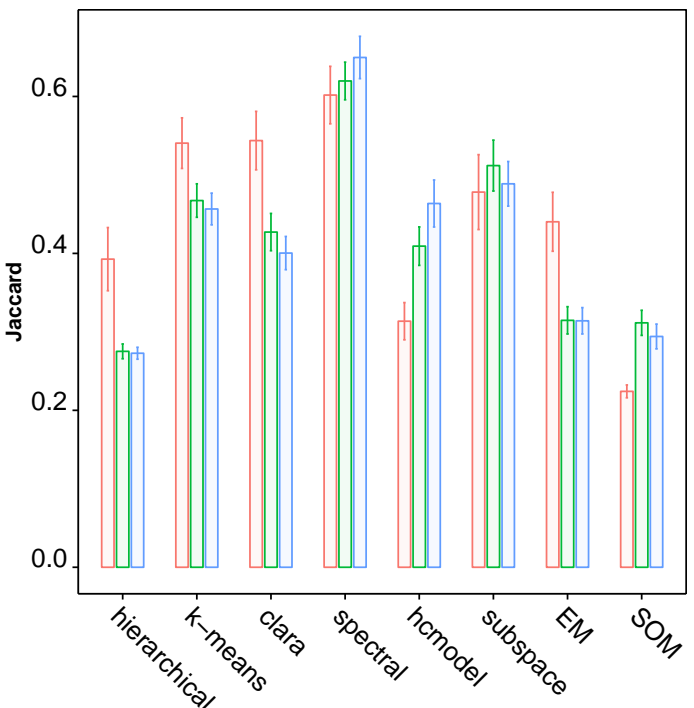

Algorithm

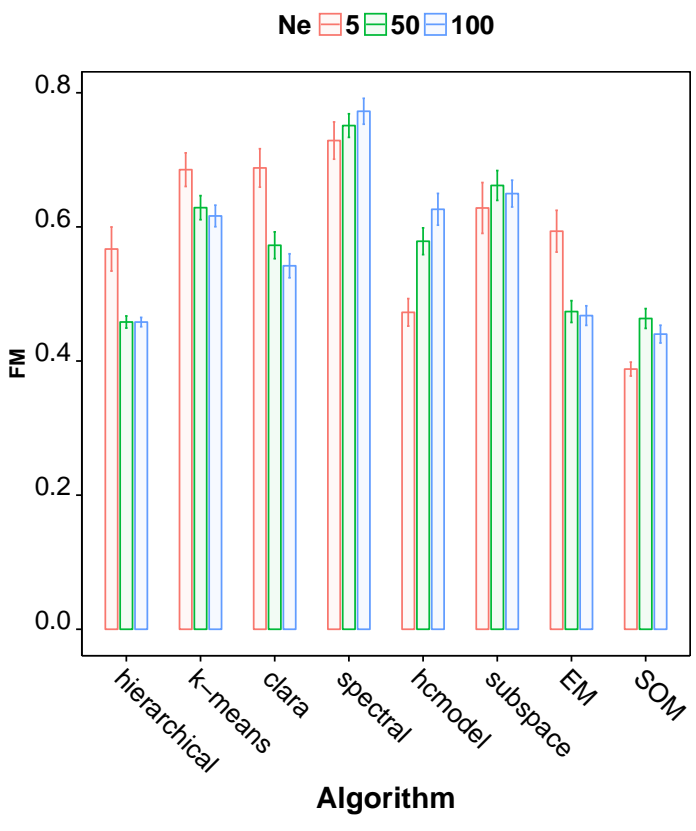

$\mathrm{Ne} \boxminus 5 \boxminus 50 \boxminus 100$

Figure 10 - Average performance of the seven considered clustering algorithms according to the number of objects per class in the dataset. All datasets described in Section 3.3 were used for evaluation. The averages were calculated separately for datasets containing 5, 50 and 100 objects per class. The considered performance indexes are (a) adjusted Rand, (b) Jaccard, (c) normalized mutual information and (d) Fowlkes Mallows.

\subsubsection{One-dimensional analysis}

The objective of the one-dimensional analysis is to verify how sensitive the accuracy of the clustering algorithms is to the variation of a single parameter. In addition, this analysis is also useful to verify whether a very simple optimization strategy can lead to significant improvements in performance. For the one-dimensional analysis, we considered the databases DB2C2F Dataset with 2 classes and 2 feature (DB2C2F) (with $\alpha=2.5$ ), DB10C2F Dataset with 10 classes and 2 
Table 1 - Average difference of accuracies obtained when clustering algorithms are used with their default configuration of parameters. In general, the spectral algorithm provides the highest accuracy rate among all evaluated methods.

\begin{tabular}{|c|c|c|c|c|c|c|c|c|c|c|}
\hline \multirow{9}{*}{ NMI } & Algorithm & hierarchical & k-means & clara & spectral & hcmodel & subspace & EM & SOM & MAcc \\
\hline & hierarchical & - & $-28.59 \%$ & $-25.89 \%$ & $-42.10 \%$ & $-22.65 \%$ & $-16.72 \%$ & $-10.29 \%$ & $-10.23 \%$ & $40.99 \%$ \\
\hline & k-means & $28.59 \%$ & - & $2.70 \%$ & $-13.51 \%$ & $5.94 \%$ & $11.87 \%$ & $18.30 \%$ & $18.36 \%$ & $69.58 \%$ \\
\hline & clara & $25.89 \%$ & $-2.70 \%$ & - & $-16.21 \%$ & $3.24 \%$ & $9.17 \%$ & $15.60 \%$ & $15.66 \%$ & $66.88 \%$ \\
\hline & spectral & $42.10 \%$ & $13.51 \%$ & $16.21 \%$ & - & $19.45 \%$ & $25.38 \%$ & $31.81 \%$ & $31.87 \%$ & $83.09 \%$ \\
\hline & hcmodel & $22.65 \%$ & $-5.94 \%$ & $-3.24 \%$ & $-19.45 \%$ & - & $5.93 \%$ & $12.36 \%$ & $12.42 \%$ & $63.64 \%$ \\
\hline & subspace & $16.72 \%$ & $-11.87 \%$ & $-9.17 \%$ & $-25.38 \%$ & $-5.93 \%$ & - & $6.43 \%$ & $6.49 \%$ & $57.71 \%$ \\
\hline & EM & $10.29 \%$ & $-18.30 \%$ & $-15.60 \%$ & $-31.81 \%$ & $-12.36 \%$ & $-6.43 \%$ & - & $0.06 \%$ & $51.28 \%$ \\
\hline & SOM & $10.23 \%$ & $-18.36 \%$ & $-15.66 \%$ & $-31.87 \%$ & $-12.42 \%$ & $6.49 \%$ & $-0.06 \%$ & $0.00 \%$ & $51.22 \%$ \\
\hline \multirow[t]{8}{*}{ ARI } & hierarchical & - & $-31.58 \%$ & $-29.06 \%$ & $-46.82 \%$ & $-18.27 \%$ & $-30.42 \%$ & $-10.07 \%$ & $-6.07 \%$ & $21.34 \%$ \\
\hline & k-means & $31.58 \%$ & - & $2.52 \%$ & $-15.24 \%$ & $13.31 \%$ & $1.16 \%$ & $21.51 \%$ & $25.51 \%$ & $52.92 \%$ \\
\hline & clara & $29.06 \%$ & $-2.52 \%$ & - & $-17.76 \%$ & $10.79 \%$ & $-1.36 \%$ & $18.99 \%$ & $22.99 \%$ & $50.40 \%$ \\
\hline & spectral & $46.82 \%$ & $15.24 \%$ & $17.76 \%$ & - & $28.55 \%$ & $16.40 \%$ & $36.75 \%$ & $40.75 \%$ & $68.16 \%$ \\
\hline & hcmodel & $18.27 \%$ & $-13.31 \%$ & $-10.79 \%$ & $-28.55 \%$ & - & $-12.15 \%$ & $8.20 \%$ & $12.20 \%$ & $39.61 \%$ \\
\hline & subspace & $30.42 \%$ & $-1.16 \%$ & $1.36 \%$ & $-16.40 \%$ & $12.15 \%$ & - & $20.35 \%$ & $24.35 \%$ & $51.76 \%$ \\
\hline & EM & $10.07 \%$ & $-21.51 \%$ & $-18.99 \%$ & $-36.75 \%$ & $-8.20 \%$ & $-20.35 \%$ & - & $4.0 \%$ & $31.41 \%$ \\
\hline & SOM & $6.07 \%$ & $-25.51 \%$ & $-22.99 \%$ & $-40.75 \%$ & $-12.20 \%$ & $-24.35 \%$ & $-4.00 \%$ & $0.00 \%$ & $27.41 \%$ \\
\hline \multirow[t]{8}{*}{ Jaccard } & hierarchical & - & $-17.46 \%$ & $-14.36 \%$ & $-30.97 \%$ & $-8.20 \%$ & $-17.94 \%$ & $-2.93 \%$ & $2.92 \%$ & $31.95 \%$ \\
\hline & k-means & $17.46 \%$ & - & $3.10 \%$ & $-13.51 \%$ & $9.26 \%$ & $-0.48 \%$ & $14.53 \%$ & $20.38 \%$ & $48.81 \%$ \\
\hline & clara & $14.36 \%$ & $-3.10 \%$ & - & $-16.61 \%$ & $6.16 \%$ & $-3.58 \%$ & $11.43 \%$ & $17.28 \%$ & $45.71 \%$ \\
\hline & spectral & $30.97 \%$ & $13.51 \%$ & $16.61 \%$ & - & $22.77 \%$ & $13.03 \%$ & $28.04 \%$ & $33.89 \%$ & $62.32 \%$ \\
\hline & hcmodel & $8.20 \%$ & $-9.26 \%$ & $-6.16 \%$ & $-22.77 \%$ & - & $-9.74 \%$ & $5.27 \%$ & $11.12 \%$ & $39.55 \%$ \\
\hline & subspace & $17.94 \%$ & $0.48 \%$ & $3.58 \%$ & $-13.03 \%$ & $9.74 \%$ & - & $15.01 \%$ & $20.86 \%$ & $49.24 \%$ \\
\hline & EM & $2.93 \%$ & $-14.53 \%$ & $-11.43 \%$ & $-28.04 \%$ & $-5.27 \%$ & $-15.01 \%$ & - & $5.85 \%$ & $34.28 \%$ \\
\hline & SOM & $-2.92 \%$ & $-20.38 \%$ & $-17.28 \%$ & $-33.89 \%$ & $-11.12 \%$ & $-20.86 \%$ & $-5.85 \%$ & $0.00 \%$ & $28.43 \%$ \\
\hline \multirow[t]{8}{*}{ FM } & hierarchical & - & $-14.98 \%$ & $-10.64 \%$ & $-25.57 \%$ & $-6.47 \%$ & $-15.22 \%$ & $-0.41 \%$ & $5.75 \%$ & $49.44 \%$ \\
\hline & k-means & $14.90 \%$ & - & $4.26 \%$ & $-10.67 \%$ & $8.43 \%$ & $-0.32 \%$ & $14.49 \%$ & $20.65 \%$ & $64.34 \%$ \\
\hline & clara & $10.64 \%$ & $-4.26 \%$ & - & $-14.93 \%$ & $4.17 \%$ & $-4.58 \%$ & $10.23 \%$ & $16.39 \%$ & $60.08 \%$ \\
\hline & spectral & $25.57 \%$ & $10.67 \%$ & $14.93 \%$ & - & $19.10 \%$ & $10.35 \%$ & $25.16 \%$ & $31.32 \%$ & $75.01 \%$ \\
\hline & hcmodel & $6.47 \%$ & $-8.43 \%$ & $-4.17 \%$ & $-19.10 \%$ & - & $-8.75 \%$ & $6.06 \%$ & $12.22 \%$ & $55.91 \%$ \\
\hline & subspace & $15.22 \%$ & $0.32 \%$ & $4.58 \%$ & $-10.35 \%$ & 8.75 & - & $14.81 \%$ & $20.97 \%$ & $64.66 \%$ \\
\hline & EM & $0.41 \%$ & $-14.49 \%$ & $-10.23 \%$ & $-25.16 \%$ & $-6.06 \%$ & $-14.81 \%$ & . & $6.16 \%$ & $49.85 \%$ \\
\hline & SOM & $-5.75 \%$ & $-20.65 \%$ & $-16.39 \%$ & $-31.32 \%$ & $-12.22 \%$ & $-20.97 \%$ & $-6.16 \%$ & - & $43.69 \%$ \\
\hline
\end{tabular}

feature (DB10C2F) (with $\alpha=4.3$ ), DB2C10F Dataset with 2 classes and 10 features (DB2C10F) (with $\alpha=1.16$ ) and DB10C10F Dataset with 10 classes and 10 features (DB10C10F) (with $\alpha=1.75$ ). For each parameter, we varied its values while keeping the other parameters value in their default configuration. The effect of varying the values of a single parameter $P$ was quantified by comparing the obtained accuracy $\Gamma(x)$ when the parameter takes the value $x$ and the accuracy $\Gamma_{\text {def }}$ achieved with the default configuration of parameters. The improvement in performance was quantified in terms of the average $(\langle S\rangle)$ and the maximum value $(\max S)$, given by

$$
\begin{gathered}
\langle S\rangle=\frac{1}{n_{P}} \sum_{x}\left(\Gamma(x)-\Gamma_{\mathrm{def}}\right), \\
\max S=\max _{x}\left(\Gamma(x)-\Gamma_{\mathrm{def}}\right),
\end{gathered}
$$

where . We also measured the sensitivity of varying the values of $P$ using the standard deviation $\Delta S:$

$$
\Delta S=\left[\frac{1}{n_{P}} \sum_{x}\left(\Gamma(x)-\Gamma_{\mathrm{def}}-\langle S\rangle\right)^{2}\right]^{1 / 2} .
$$

In addition to the aforementioned quantities, we also measured, for each dataset, the maximum accuracy obtained when varying each single parameter of the algorithm. We then calculate the average of maximum accuracies, $\langle\max A c c\rangle$, obtained over all considered datasets. In Table 2, we show the values of $\langle S\rangle, \max S, \Delta S$ and $\langle\max A c c\rangle$ for datasets containing two features. 
When considering a two-class problem (DB2C2F), a significant improvement in performance $(\langle S\rangle=10.75 \%$ and $\langle S\rangle=13.35 \%)$ was observed when varying parameter modelName, minPts and kpar of, respectively, the EM and spectral methods. For all other cases, only minor average gain in performance was observed. For the 10-class problem, we notice that an inadequate value for parameter method of the hierarchical algorithm can lead to much worse accuracy $(16.15 \%$ on average). In most cases, however, the average variation in performance was small.

Table 2 - One-parameter analysis performed in DB2C2F and DB10C2F. This analysis is based on the performance (measured through the ARI index) obtained when varying a single parameter of the clustering algorithm while maintaining the others in their default configuration. $\langle S\rangle, \max S$, $\Delta S$ is associated with the average, standard deviation and maximum difference between the performance obtained when varying a single parameter and the performance obtained for the default parameter values. We also measure $\langle\max A c c\rangle$, the average of best ARI values obtained when varying each parameter, where the average is calculated over all considered datasets.

\begin{tabular}{|c|c|c|c|c|c|c|c|c|c|}
\hline \multirow[b]{2}{*}{ Algorithm } & \multirow[b]{2}{*}{ Parameter } & \multicolumn{4}{|c|}{ DB2C2F } & \multicolumn{4}{|c|}{ DB10C2F } \\
\hline & & $\begin{array}{l}\langle S\rangle \\
(\%)\end{array}$ & $\begin{array}{l}\Delta S \\
(\%)\end{array}$ & $\begin{array}{c}\max S \\
(\%)\end{array}$ & $\begin{array}{c}\langle\max A c c\rangle \\
(\%)\end{array}$ & $\begin{array}{l}\langle S\rangle \\
(\%)\end{array}$ & $\begin{array}{c}\Delta S \\
(\%)\end{array}$ & $\begin{array}{c}\max S \\
(\%)\end{array}$ & $\begin{array}{c}\langle\max A c c\rangle \\
(\%)\end{array}$ \\
\hline k-means & iter.max & 0.05 & 2.37 & 14.46 & 51.5 & 0.04 & 0.91 & 4.49 & 47.3 \\
\hline $\mathrm{k}$-means & nstart & 1.98 & 5.62 & 16.73 & 51.9 & 1.24 & 1.98 & 6.80 & 47.9 \\
\hline $\mathrm{k}$-means & algorithm & 0.29 & 2.46 & 6.63 & 49.8 & -0.92 & 1.29 & 0.65 & 45.0 \\
\hline clara & metric & -1.52 & 8.10 & 11.27 & 49.6 & -3.66 & 5.36 & 5.10 & 42.5 \\
\hline clara & samples & -0.10 & 3.82 & 6.39 & 52.3 & -0.21 & 3.03 & 7.48 & 47.5 \\
\hline clara & sampsize & -2.78 & 12.96 & 27.31 & 54.0 & -0.54 & 2.92 & 4.88 & 47.1 \\
\hline clara & rngR & -0.16 & 3.19 & 4.19 & 51.0 & -4.53 & 4.04 & -0.03 & 41.7 \\
\hline hierarchical & metric & 5.27 & 22.28 & 63.65 & 23.3 & 1.83 & 3.64 & 9.26 & 42.3 \\
\hline hierarchical & method & 2.07 & 36.90 & 100.0 & 57.2 & -16.15 & 21.26 & 15.89 & 46.5 \\
\hline hierarchical & par.method & 0.0 & 0.0 & 0.0 & 18.0 & 0.0 & 0.0 & 0.0 & 40.5 \\
\hline spectral & kernel & -0.61 & 10.42 & 39.45 & 43.7 & -0.3 & 2.84 & 6.78 & 48.0 \\
\hline spectral & kpar & 7.36 & 16.78 & 33.3 & 44.6 & -1.83 & 3.16 & 3.35 & 43.5 \\
\hline spectral & iter & 1.14 & 19.19 & 85.34 & 54.1 & 0.06 & 2.62 & 5.84 & 47.9 \\
\hline spectral & mod.simple & -2.11 & 9.7 & 33.32 & 43.0 & 0.54 & 2.02 & 4.5 & 47.7 \\
\hline hcmodel & modelName & -2.41 & 19.48 & 29.89 & 60.0 & -0.56 & 3.26 & 6.44 & 48.4 \\
\hline hcmodel & use & -2.14 & 10.14 & 12.58 & 57.4 & -0.50 & 1.11 & 2.19 & 47.5 \\
\hline EM & $\mathrm{Z}$ & 1.71 & 8.77 & 19.34 & 33.9 & 7.04 & 8.30 & 28.17 & 45.4 \\
\hline EM & modelName & 10.75 & 26.18 & 66.64 & 64.4 & 0.14 & 6.25 & 16.20 & 45.0 \\
\hline SOM & $\operatorname{dim}$ & -2.63 & 8.22 & 20.89 & 48.6 & -0.01 & 2.8 & 6.57 & 40.9 \\
\hline SOM & init & 2.79 & 11.9 & 43.57 & 45.4 & 0.56 & 4.41 & 11.66 & 37.1 \\
\hline SOM & alpha & 1.66 & 6.65 & 20.23 & 45.9 & -0.91 & 1.72 & 2.15 & 36.6 \\
\hline SOM & neigh & -3.95 & 6.18 & 16.53 & 37.2 & 3.26 & 1.82 & 6.67 & 39.8 \\
\hline SOM & topl & -3.4 & 6.56 & 16.53 & 37.4 & -0.03 & 1.52 & 2.47 & 36.5 \\
\hline SOM & radius & 4.26 & 8.14 & 26.42 & 48.5 & -5.31 & 2.61 & 0.24 & 32.4 \\
\hline SOM & rlen & 1.75 & 7.8 & 27.97 & 43.7 & 1.49 & 1.92 & 6.7 & 39.5 \\
\hline
\end{tabular}

In Table 3, we show the values of $\langle S\rangle$, $\max S, \Delta S$ and $\langle\max A c c\rangle$ for datasets described by 10 features. For the two-class clustering problem, a moderate improvement can be observed for the k-means, hierarchical and optics algorithm through the variation of, respectively, parameter nstart, method and minPts. A large increase in accuracy was observed when varying parameter modelName of the EM method. Changing the modelName used by the algorithm led to, on average, an improvement of $18.8 \%$. A similar behaviour was obtained when the number of classes was set to $C=10$. For 10 classes, the variation of method in the hierarchical algorithm provided an average improvement of $6.72 \%$. A high improvement was also observed when varying parameter modelName of the EM algorithm, with an average improvement of $13.63 \%$.

In contrast to the parameters discussed so far, the variation of some parameters plays a 
minor role in the discriminative power of the clustering algorithms. This is the case, for instance, of parameters kernel and iter of the spectral clustering algorithm and parameter itermax of the $\mathrm{k}$-means clustering. In some cases, the effect of a unidimensional variation of parameter resulted in a reduction of performance. For instance, the variation of min.individuals and models of the subspace algorithm provided an average loss of accuracy on the order of $\langle S\rangle=20 \%$, depending on the dataset. Parameters metric and $r n g R$ of the Clara algorithm also led to marked decrease in performance.

Table 3 - One-parameter analysis performed in DB2C10F and DB10C10F. This analysis is based on the performance obtained when varying a single parameter, while maintaining the others in their default configuration. $\langle S\rangle, \max S, \Delta S$ are associated with the average, standard deviation and maximum difference between the performance obtained when varying a single parameter and the performance obtained for the default parameter values. We also measure $\langle\max A c c\rangle$, the average of best ARI values obtained when varying each parameter, where the average is calculated over all considered datasets.

\begin{tabular}{|c|c|c|c|c|c|c|c|c|c|}
\hline \multirow[b]{2}{*}{ Algorithm } & \multirow[b]{2}{*}{ Parameter } & \multicolumn{4}{|c|}{ DB2C10F } & \multicolumn{4}{|c|}{ DB10C10F } \\
\hline & & $\begin{array}{l}\langle S\rangle \\
(\%)\end{array}$ & $\begin{array}{c}\Delta S \\
(\%)\end{array}$ & $\begin{array}{c}\max S \\
(\%)\end{array}$ & $\begin{array}{c}\langle\max A c c\rangle \\
(\%)\end{array}$ & $\begin{array}{l}\langle S\rangle \\
(\%)\end{array}$ & $\begin{array}{l}\Delta S \\
(\%)\end{array}$ & $\begin{array}{c}\max S \\
(\%)\end{array}$ & $\begin{array}{c}\langle\max A c c\rangle \\
(\%)\end{array}$ \\
\hline k-means & iter.max & 0.30 & 8.13 & 36.36 & 53.2 & 0.14 & 1.92 & 6.41 & 56.6 \\
\hline k-means & nstart & 5.26 & 12.0 & 36.36 & 53.5 & 2.68 & 2.65 & 9.43 & 57.8 \\
\hline k-means & algorithm & -0.35 & 6.72 & 25.5 & 42.3 & -2.11 & 3.3 & 2.71 & 52.7 \\
\hline clara & metric & -10.9 & 22.31 & 25.05 & 51.8 & -16.63 & 6.84 & -5.1 & 37.6 \\
\hline clara & samples & 1.04 & 8.94 & 25.05 & 60.4 & -4.83 & 8.96 & 10.26 & 51.9 \\
\hline clara & sampsize & 0.44 & 13.94 & 37.31 & 61.0 & -4.46 & 9.97 & 14.18 & 57.6 \\
\hline clara & rngR & -2.89 & 15.08 & 25.05 & 56.9 & -14.75 & 6.29 & -5.21 & 39.3 \\
\hline hierarchical & metric & 4.82 & 21.46 & 96.0 & 9.7 & 1.15 & 8.52 & 27.18 & 19.2 \\
\hline hierarchical & method & 8.76 & 21.93 & 100.0 & 43.7 & 6.72 & 25.52 & 71.1 & 61.5 \\
\hline hierarchical & par.method & 0.00 & 0.00 & 0.00 & 0.00 & 0.0 & 0.0 & 0.0 & 13.8 \\
\hline spectral & kernel & 0.64 & 15.91 & 50.56 & 87.9 & 1.3 & 7.13 & 15.81 & 82.3 \\
\hline spectral & kpar & -1.08 & 16.88 & 50.56 & 88.1 & -2.25 & 5.81 & 6.03 & 71.7 \\
\hline spectral & iter & -0.96 & 15.91 & 50.56 & 87.9 & 0.45 & 7.27 & 20.01 & 79.8 \\
\hline spectral & mod.simple & 3.36 & 15.72 & 50.56 & 87.9 & -1.35 & 7.55 & 14.24 & 78.7 \\
\hline subspace & models & -1.77 & 36.80 & 97.4 & 100.0 & -22.44 & 8.92 & -6.7 & 69.6 \\
\hline subspace & init & -0.78 & 23.47 & 97.4 & 99.5 & -0.57 & 9.29 & 11.13 & 87.4 \\
\hline subspace & algo & -1.32 & 1.99 & 0.27 & 88.9 & 0.7 & 1.1 & 1.9 & 87.4 \\
\hline subspace & min.individuals & -26.9 & 43.17 & 10.73 & 90.9 & -12.32 & 16.6 & 7.78 & 89.1 \\
\hline hcmodel & modelName & 3.70 & 24.23 & 75.6 & 51.3 & 3.63 & 4.89 & 14.4 & 61.5 \\
\hline hcmodel & use & -0.92 & 17.68 & 51.47 & 49.1 & -1.86 & 6.09 & 10.69 & 55.9 \\
\hline EM & $\mathrm{Z}$ & 1.68 & 8.62 & 18.99 & 29.9 & -0.35 & 5.49 & 15.06 & 43.3 \\
\hline EM & modelName & 18.80 & 31.93 & 96.62 & 100.0 & 13.63 & 16.09 & 64.52 & 91.6 \\
\hline SOM & $\operatorname{dim}$ & -9.69 & 12.27 & 16.5 & 31.5 & 1.35 & 2.64 & 10.99 & 37.4 \\
\hline SOM & init & -6.86 & 14.84 & 25.09 & 27.8 & -2.86 & 4.46 & 2.07 & 32.7 \\
\hline SOM & alpha & -5.9 & 10.71 & 9.95 & 26.8 & -1.68 & 2.43 & 2.71 & 33.2 \\
\hline SOM & neigh & -13.17 & 12.61 & 7.09 & 14.5 & 1.43 & 3.68 & 13.02 & 35.2 \\
\hline SOM & topl & -13.18 & 12.63 & 7.09 & 14.4 & 0.92 & 2.07 & 7.01 & 33.8 \\
\hline SOM & radius & 7.38 & 29.83 & 91.14 & 76.1 & -0.71 & 1.83 & 2.07 & 32.8 \\
\hline SOM & rlen & -2.22 & 12.75 & 20.57 & 32.5 & 2.68 & 3.56 & 11.61 & 38.2 \\
\hline
\end{tabular}

\subsubsection{Multi-dimensional analysis}

A complete analysis of the performance of a clustering algorithm requires the simultaneous variation of all of its parameters. Nevertheless, such a task is difficult to do in practice, given a large number of parameter combinations that need to be taken into account. Therefore, here we consider a random variation of parameters aimed at obtaining a sampling of each algorithm performance for its complete multi-dimensional parameter space. 
The methodology is applied as follows. Considering the one-dimensional variation of parameters, presented in the previous section, we identify the parameter bounds, $\left[P_{\min }, P_{\max }\right]$, where the classification either does not significantly change or provides much worse results when compared to the default parameter value. Such bounds define the interval where the parameter will be randomly sampled. In order to generate the values for a given set of parameters $P^{(1)}, P^{(2)}, \ldots, P^{(n)}$ of an algorithm, we randomly sample each parameter $P^{(i)}$ according to a uniform distribution defined in the interval $\left[P_{\min }^{(i)}, P_{\max }^{(i)}\right]$. This procedure generates sets of parameter values, which are then used to evaluate the performance of the algorithms. For each algorithm, 500 sets of parameters were generated.

The performance of the algorithms for the different sets of parameters was evaluated according to the following procedure. Consider the histogram of ARI values obtained for the random sampling of parameters for the SOM algorithm, shown in Figure 11. The red dashed line indicates the ARI value obtained for the default parameters of the algorithm. The light blue shaded region indicates the parameters configurations where the performance of the algorithm improved. From this result, we calculated four main measures. The first, which we call p-value, is given by the area of the blue region divided by the total histogram area, multiplied by 100 in order to result in a percentage value. The $\mathrm{p}$-value represents the percentage of parameter configurations where the algorithm performance improved when compared to the default parameters configuration. The second, third and fourth measures are given by the mean, $\langle R\rangle$, standard deviation, $\Delta R$, and maximum value, $\max R$, of the relative performance for all cases where the performance is improved (e.g. the blue shaded region in Figure 11). The relative performance is calculated as the difference in performance between a given realization of parameter values and the default parameters. The mean indicates the expected improvement of the algorithm for the random variation of parameters. The standard deviation represents the stability of such improvement, that is, how certain one is that the performance will be improved when doing such random variation. The maximum value indicates the largest improvement obtained when random parameters are considered. We also measured the average of the maximum accuracies $\langle$ max ARI $\rangle$ obtained for each dataset when randomly selecting the parameters.

In Table 4 we show the performance (ARI) of the algorithms for dataset DB2C2F when applying the aforementioned random selection of parameters. The EM method is the only algorithms with a p-value larger than $50 \%$. Also, a high average gain in performance was observed for the EM (22.1\%) and hierarchical (30.6\%) methods. Moderate improvement was obtained for the homodel, k-means, spectral and SOM.

The performance of the algorithms for dataset DB10C2F is presented in Table 5. A high p-value was obtained for EM (76.5\%) and k-means (77.7\%) and SOM (62.9\%). Nevertheless, the average improvement in performance was relatively low for the most of the algorithms.

A more diverse variation in performance was observed for dataset $\mathrm{DB} 2 \mathrm{C} 10 \mathrm{~F}$, with results shown in Table 6. The EM, k-means and hierarchical clustering algorithms resulted in a p- 

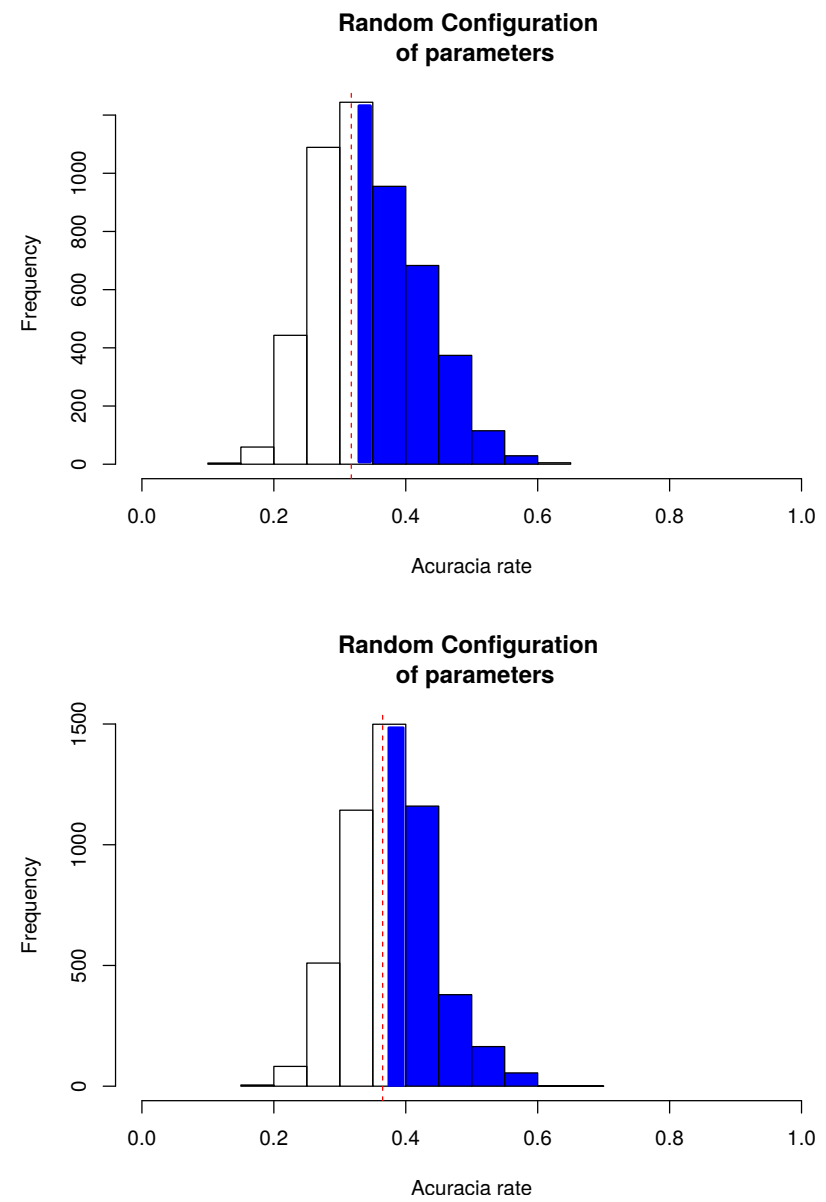

Figure 11 - Distribution of ARI values obtained for the random sampling of the SOM parameters. The algorithm was applied to dataset DB10C10F and DB10C2F, and 500 sets of parameters were drawn.

value larger than $50 \%$. In such cases, when the performance was improved, the average gain in performance was, respectively, $30.1 \%, 18.0 \%$ and $25.9 \%$. This means that the random variation of parameters might represent a valid approach for improving these algorithms. Actually, with the exception of Clara, all methods display significant average improvement in performance for this dataset. The results also show that a maximum accuracy of $100 \%$ can be achieved for the EM and subspace algorithms.

In Table 7 we show the performance of the algorithms for dataset DB10C10F. The p-values for the EM, Clara, k-means and SOM indicate that the random selection of parameters usually improves the performance of these algorithms. The hierarchical algorithm can be significantly improved by the considered random selection of parameters. This is a consequence of the default value of parameter method, which, as discussed in Section 3.5.2, is not appropriate for this dataset. 
Table 4 - Multi-parameter analysis performed in dataset DB2C2F. The p-value represents the probability that the classifier set with a random configuration of parameters outperform the same classifier set with its default parameters. $\langle R\rangle, \Delta R$ and $\max R$ represent the average, standard deviation and maximum value of the improvement obtained when random parameters are considered. Column $\langle\max A R I\rangle$ indicates the average of the best accuracies obtained for each dataset.

\begin{tabular}{lccccc}
\hline \hline Algorithm & $\begin{array}{c}\mathrm{p} \text {-value } \\
(\%)\end{array}$ & $\begin{array}{c}\langle R\rangle \\
(\%)\end{array}$ & $\begin{array}{c}\Delta R \\
(\%)\end{array}$ & $\begin{array}{c}\max R \\
(\%)\end{array}$ & $\begin{array}{c}\langle\max \text { ARI }\rangle \\
(\%)\end{array}$ \\
\hline EM & 68.1 & 22.1 & 21.7 & 69.6 & 69.0 \\
hierarchical & 43.9 & 30.6 & 33.6 & 100.0 & 63.0 \\
clara & 29.2 & 4.9 & 4.7 & 27.3 & 60.0 \\
hcmodel & 25.8 & 13.3 & 8.2 & 29.9 & 63.0 \\
k-means & 21.7 & 13.2 & 3.9 & 21.4 & 55.0 \\
spectral & 47.0 & 14.7 & 13.9 & 85.3 & 59.0 \\
SOM & 49.0 & 13.1 & 12.5 & 55.1 & 72.1 \\
\hline
\end{tabular}

Table 5 - Multi-parameter analysis performed in dataset DB10C2F. The p-value represents the probability that the classifier set with a random configuration of parameters outperform the same classifier set with its default parameters. $\langle R\rangle, \Delta R$ and $\max R$ represent the average, standard deviation and maximum value of the improvement obtained when random parameters are considered. Column $\langle$ max ARI $\rangle$ indicates the average of the best accuracies obtained for each dataset.

\begin{tabular}{lccccc}
\hline \hline Algorithm & $\begin{array}{c}\text { p-value } \\
(\%)\end{array}$ & $\begin{array}{c}\langle R\rangle \\
(\%)\end{array}$ & $\begin{array}{c}\Delta R \\
(\%)\end{array}$ & $\begin{array}{c}\max R \\
(\%)\end{array}$ & $\begin{array}{c}\langle\max \text { ARI }\rangle \\
(\%)\end{array}$ \\
\hline \hline EM & 76.5 & 7.5 & 8.0 & 35.8 & 51.4 \\
clara & 54.7 & 2.3 & 1.8 & 9.0 & 51.0 \\
k-means & 77.7 & 2.2 & 1.7 & 6.9 & 49.0 \\
hcmodel & 28.4 & 2.7 & 2.5 & 6.8 & 49.0 \\
hierarchical & 36.6 & 5.9 & 4.2 & 21.7 & 49.0 \\
spectral & 40.0 & 2.3 & 1.6 & 8.0 & 52.0 \\
SOM & 62.9 & 3.7 & 2.7 & 18.8 & 48.7 \\
\hline
\end{tabular}

Table 6 - Multi-parameter analysis performed in dataset DB2C10F. The p-value represents the probability that the classifier set with a random configuration of parameters outperform the same classifier set with its default parameters. $\langle R\rangle, \Delta R$ and $\max R$ represent the average, standard deviation and maximum value of the improvement obtained when random parameters are considered. Column $\langle\max$ ARI $\rangle$ indicates the average of the best accuracies obtained for each dataset.

\begin{tabular}{lccccc}
\hline \hline Algorithm & $\begin{array}{c}\text { p-value } \\
(\%)\end{array}$ & $\begin{array}{c}\langle R\rangle \\
(\%)\end{array}$ & $\begin{array}{c}\Delta R \\
(\%)\end{array}$ & $\begin{array}{c}\max R \\
(\%)\end{array}$ & $\begin{array}{c}\langle\max \text { ARI }\rangle \\
(\%)\end{array}$ \\
\hline EM & 70.8 & 30.1 & 29.9 & 96.6 & 100.0 \\
hierarchical & 52.0 & 25.9 & 31.4 & 100.0 & 80.0 \\
subspace & 11.1 & 43.1 & 45.4 & 97.4 & 100.0 \\
clara & 44.9 & 6.5 & 6.3 & 37.3 & 70.0 \\
hcmodel & 38.4 & 31.8 & 25.3 & 81.2 & 70.0 \\
k-means & 50.1 & 18.0 & 7.1 & 62.4 & 60.0 \\
spectral & 48.9 & 9.9 & 18.5 & 31.5 & 90.0 \\
SOM & 43.9 & 18.7 & 15.2 & 83.3 & 79.3 \\
\hline
\end{tabular}


Table 7 - Multi-parameter analysis performed in dataset DB10C10F. The p-value represents the probability that the classifier set with a random configuration of parameters outperform the same classifier set with its default parameters. $\langle R\rangle, \Delta R$ and $\max R$ represent the average, standard deviation and maximum value of the improvement obtained when random parameters are considered. Column $\langle\max$ ARI $\rangle$ indicates the average of the best accuracies obtained for each dataset.

\begin{tabular}{lccccc}
\hline \hline Algorithm & $\begin{array}{c}\text { p-value } \\
(\%)\end{array}$ & $\begin{array}{c}\langle R\rangle \\
(\%)\end{array}$ & $\begin{array}{c}\Delta R \\
(\%)\end{array}$ & $\begin{array}{c}\max R \\
(\%)\end{array}$ & $\begin{array}{c}\langle\max \text { ARI }\rangle \\
(\%)\end{array}$ \\
\hline \hline EM & 86.0 & 17.1 & 15.5 & 69.1 & 100.0 \\
clara & 72.1 & 7.1 & 4.4 & 22.8 & 68.0 \\
k-means & 83.0 & 4.3 & 2.3 & 12.0 & 60.0 \\
hcmodel & 53.4 & 7.4 & 4.6 & 17.5 & 64.0 \\
hierarchical & 51.9 & 32.1 & 19.4 & 72.9 & 68.0 \\
spectral & 49.1 & 5.6 & 4.1 & 19.7 & 87.3 \\
subspace & 10.7 & 7.5 & 4.7 & 21.4 & 99.3 \\
SOM & 64.2 & 6.4 & 4.7 & 28.4 & 52.7 \\
\hline
\end{tabular}

\subsection{Conclusions}

The analysis of the advantages and pitfalls of clustering algorithms is also a difficult task that has been received much attention from the research community. Here, we approached this task focusing on a comprehensive methodology for generating a large diversity of heterogeneous datasets with precisely defined properties such as the distances between classes and correlations between features. Using packages from the $\mathrm{R}$ language, we developed a comparison of the performance of seven popular clustering methods applied to 270 artificial datasets. Three situations were considered: default parameters, single parameter variation and random variation of parameters. Besides serving as a practical guide to the application of clustering methods when the researcher is not an expert in data mining techniques, a number of interesting results regarding the considered clustering methods were obtained.

Regarding the default parameters, the difference in performance of clustering methods was not significant for low-dimensional datasets. Specifically, the Kruskal-Wallis test on the differences in performance when 2 features were considered resulted in a p-value of $p=0.00017$, with a chi-squared distance of $\chi^{2}=21.12$. For 10 features, a p-value of $p=2.06 \times 10^{-7}$ $\left(\chi^{2}=44.08\right)$ was obtained. Considering 50 features resulted in a $\mathrm{p}$-value of $p=3.59 \times 10^{-7}$ for the Kruskal-Wallis test $\left(\chi^{2}=42.83\right)$.

The Spectral method provided the best performance when using default parameters, with an Adjusted Rand Index (ARI) of $68.16 \%$, as indicated in Table 1. In contrast, the hierarchical method showed the worst results with an ARI of 21.34\%. It is also interesting that underestimating the number of classes in the dataset led to worse performance than in overestimation situations. This was observed for all algorithms and is in accordance with previous results (ERMAN; ARLITT; MAHANTI, 2006).

Regarding single parameter variations, for datasets containing 2 features, the hierarchical 
and EM methods showed significant performance variation. On the other hand, for datasets containing 10 or more features, most methods could be readily improved through changes in selected parameters.

With respect to the multidimensional analysis of datasets containing ten classes and two features, the performance of the algorithms for the multidimensional selection of parameters was similar to that using the default parameters. This suggests that the algorithms are not sensitive to parameter variations for this dataset. For datasets containing two classes and ten features, the EM, hcmodel, subspace and hierarchical algorithm showed a significant gain in performance. The EM algorithm also resulted in a high p-value (70.8\%), which indicates that many parameter values for this algorithm can provide better results than the default configuration. For datasets containing ten classes and ten features, the improvement was significantly lower for almost all the algorithms, with the exception of the hierarchical clustering.

In Tables 8 and 9 we show a summary of the best accuracies obtained during our analysis. The tables contain the best performance, measured as the ARI of the resulting partitions, achieved by each algorithm in the three considered situations (default, one- and multi-dimensional adjustment of parameters). The results are respective to datasets $\mathrm{DB} 2 \mathrm{C} 2 \mathrm{~F}, \mathrm{DB} 10 \mathrm{C} 2 \mathrm{~F}, \mathrm{DB} 2 \mathrm{C} 10 \mathrm{~F}$ and DB10C10F. We observe that, for datasets containing 2 features, the algorithms tend to show similar performance, especially when the number of classes is increased. For datasets containing 10 features or more, the spectral algorithm seems to consistently provide the best performance, although the EM, hierarchical, k-means and subspace algorithms can also achieve similar performance with some parameter tuning.

Table 8 - Summary table for the performance of clustering algorithms in datasets DB2C2F and DB10C2F. $A R I_{d e f}$ represents the average accuracy obtained when considering the default parameters of the algorithms. $A R I_{\text {best }_{p}}$ represents the average of the best accuracies obtained when varying a single parameter. $A R I_{\text {best }_{r}}$ represents the average of the best accuracies obtained when parameters are randomly selected.

\begin{tabular}{|c|c|c|c|c|c|c|c|}
\hline \multirow[b]{2}{*}{ \# } & \multirow[b]{2}{*}{ Algorithm } & \multicolumn{3}{|c|}{ DB2C2F } & \multicolumn{3}{|c|}{ DB10C2F } \\
\hline & & $\begin{array}{c}A R I_{d e f} \\
(\%)\end{array}$ & $\begin{array}{c}A R I_{\text {best }_{p}} \\
(\%)\end{array}$ & $\begin{array}{c}A R I_{\text {best }} \\
(\%)\end{array}$ & $\begin{array}{c}A R I_{d e f} \\
(\%)\end{array}$ & $\begin{array}{c}A R I_{\text {best }} \\
(\%)\end{array}$ & $\begin{array}{c}A R I_{\text {best }_{r}} \\
(\%)\end{array}$ \\
\hline 3 & EM & 32.2 & 64.4 & 69.0 & 38.4 & 45.4 & $\begin{array}{l}51.4 \\
\end{array}$ \\
\hline 2 & spectral & 37.2 & 54.1 & 59.0 & 45.4 & 48.0 & 52.0 \\
\hline 4 & clara & 51.1 & 54.0 & 60.0 & 46.2 & 47.5 & 51.0 \\
\hline 5 & hcmodel & 54.0 & 60.0 & 63.0 & 47.1 & 48.4 & 49.0 \\
\hline 6 & k-means & 48.1 & 51.9 & 55.0 & 45.2 & 47.9 & 49.0 \\
\hline 7 & hierarchical & 18.0 & 57.2 & 63.0 & 40.5 & 46.5 & 49.0 \\
\hline 8 & SOM & 38.1 & 48.6 & 72.1 & 36.50 & 40.9 & 48.7 \\
\hline
\end{tabular}


Table 9 -Summary table for the performance of clustering algorithms in datasets DB2C10F and DB10C10F. $A R I_{d e f}$ represents the average accuracy obtained when considering the default parameters of the algorithms. $A R I_{\text {best }_{p}}$ represents the average of the best accuracies obtained when varying a single parameter. $A R I_{\text {best }_{r}}$ represents the average of the best accuracies obtained when parameters are randomly selected.

\begin{tabular}{|c|c|c|c|c|c|c|c|}
\hline & & \multicolumn{3}{|c|}{ DB2C10F } & \multicolumn{3}{|c|}{ DB10C10F } \\
\hline \# & Algorithm & $\begin{array}{c}A R I_{d e f} \\
(\%)\end{array}$ & $\begin{array}{c}A R I_{\text {best }} \\
(\%)\end{array}$ & $\begin{array}{c}A R I_{\text {best }} \\
(\%)\end{array}$ & $\begin{array}{c}A R I_{d e f} \\
(\%)\end{array}$ & $\begin{array}{c}A R I_{\text {best }} \\
(\%)\end{array}$ & $\begin{array}{c}A R I_{\text {best }} \\
(\%)\end{array}$ \\
\hline 1 & subspace & 89.9 & 100.0 & 100.0 & 86.1 & 89.1 & 99.3 \\
\hline 3 & EM & 23.4 & 100.0 & 100.0 & 40.9 & 91.6 & 100.0 \\
\hline 2 & spectral & 82.4 & 88.1 & 90.0 & 70.9 & 82.3 & 87.3 \\
\hline 4 & clara & 53.0 & 61.0 & 70.0 & 51.9 & 57.6 & 68.0 \\
\hline 5 & hcmodel & 34.2 & 51.3 & 70.0 & 54.2 & 61.5 & 64.0 \\
\hline 6 & k-means & 36.6 & 53.5 & 60.0 & 52.0 & 57.8 & 60.0 \\
\hline 7 & hierarchical & 0.0 & 43.7 & 80.0 & 13.8 & 61.5 & 68.0 \\
\hline 8 & SOM & 23.0 & 76.1 & 79.3 & 31.74 & 38.2 & 52.7 \\
\hline
\end{tabular}



CHAPTER

4

\section{THE IMPACT OF INTERCONNECTING TOPOLOGIES ON SOM NEURAL} NETWORKS

As discussed in chapter 2, the effort of the area of complex networks to characterize real networks has allowed us to know specific characteristics of networks that surround us, such as biological networks, the Internet, and transportation systems (NEWMAN, 2010b). Real networks present specific characteristics such as high clustering, small average shortest path lengths and heterogeneity of degree distribution. Classical network models such as the ER network captures only a small number of properties observed in real networks, such as low the average shortest path length, leaving out other properties. Similarly, regular networks are not accurate models to represent the structure of real-world networks, since they present nodes with the same number of connections.

In addition to the very heterogeneous structure, real-world networks present correlations between the degrees of nodes - this property is called assortativity (NEWMAN, 2003). Depending on the type of correlation, a network can be assortative (positive correlation) or disassortative (negative correlation). Studies have shown the impact of the degree of correlation in spreading of diseases and synchronization phenomena (BARRAT; BARTHELEMY; VESPIGNANI, 2008; NEWMAN, 2010b). While biological networks tend to be disassortative, social networks are often assortative. Assortative networks tend to be robust against attack.

Most studies focusing on the Kohonen network consider as a network topology, a regular network. As indicated in Chapter 2, three network models were considered in our research, small world, deactivation model and degree correlated networks. In deactivation model, a high clustering coefficient is observed whose growth mechanism, does not allow the formation of shortcuts between the deactivated region of the network. The degree correlated network allows creating networks with different assortativity values without affecting the degree distribution of 
the network.

In this section, we will first study the relation topological influence and performance of SOM network through the investigation of three different network models such as small world, deactivation model and degree correlated network that were presented in section ??. Three datasets will be used in our analysis, i.e., digits, musical symbols and letters dataset. In addition, we will study the topological characteristics of the topological map SOM considering the same models indicated above lines.

\subsection{The impact on accuracy}

In this first part of the experiments, our interest is focused on how the neuronal interconnection topology can influence the learning and accuracy of the obtained neuronal network. In section ?? we have briefly explained the network models that we are using in our experiments.

\subsubsection{Dataset}

The SOM networks were trained and tested with respect to three datasets. The first is the MNIST dataset (LECUN, 1998) wich contains digits from digit 0 to 9. For each neuron, the 784-dimensional input vector is rearranged into a $28 \times 28$ matrix. For the training and test step, 30,000 and 10,000 examples were used, respectively.

The second is the EMNIST Letters dataset (COHEN et al., 2017) wich contains uppercase and lowercase handwritten letters collected from over 500 writers, rearranged into a $28 \times 28$ matrix. For the training and test step, 26,000 and 9,646 examples were adopted.

The third database contains handwritten musical symbols (FORNÉS; LLADÓS; SÁNCHEZ, 2008) of 4100 black and white symbols of 7 different classes: flat, natural, sharp, double-sharp, c-clef, g-clef, f-clef. The musical symbols were rearranged into a $28 \times 28$ matrix. The training and test steps were performed with respect to 3075 and 1025 examples. The error rate are an averaged over ten realizations. For all experiments the map have a rectangular neighborhood shape.

\subsubsection{Analysis and results}

First, we evaluate the error rate considering the three different topologies (small world, deactivation model and degree-correlated networks) described in the chapter 2 . Then, we quantify the influence of topological measurements, such as the average path length $\langle L\rangle$ and the average clustering coefficient $\langle C C\rangle$, on the accuracy. The error rate for the test set was calculated as $E=n_{\text {error }} / N_{\text {test }}$, where $n_{\text {error }}$ is the number of misclassified elements of the test set and $N_{\text {test }}$ the 


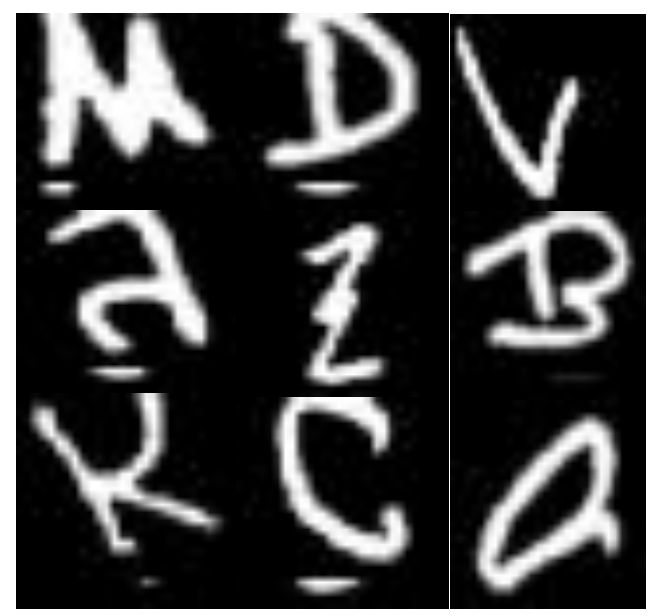

(a)

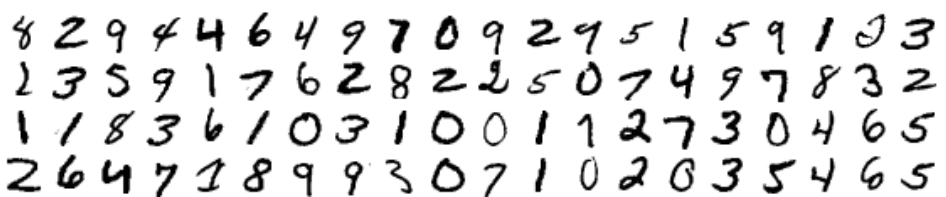

(b)
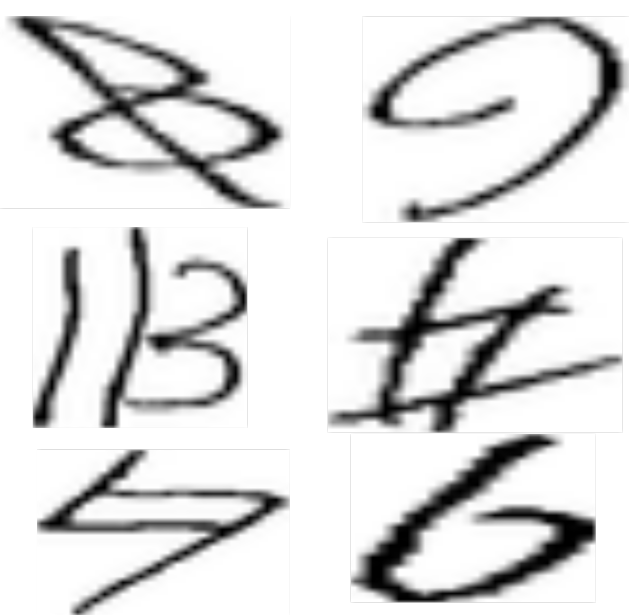

(c)

Source: Elaborated by the author.

Figure 12 - Dataset: From top to bottom correspond to the EMNIST, MNIST and musical symbols dataset. 
size of the test set. Maps of sizes 35x35 and 50x50 with $\langle k\rangle=4$ and $\sigma=15$ were used in the experiments.

The obtained results are shown in Figure 13. The first, second and third rows of Figure 13 correspond to, respectively, the digit, musical symbols and letters datasets. The plots from left to right column correspond to the small world, deactivation model and degree correlated networks. In Figure 13a we observe that, as the number of learning steps increases, the error rate for the different parameters $p$ decreases. At short scales (few learning steps), increasing $p$ leads to a large increase in the error rate. This happens because, for large $p$, almost all neurons are updated at each learning step. Similar behaviors were verified for the other datasets $13 \mathrm{~d}$ and $13 \mathrm{~g}$. In deactivation model, the error rate depends on parameter $\mu$ (as shown in Figures 13b, 13e and $13 \mathrm{~h}$ ). The lowest error rates are obtained when $\mu=0$, which defines a highly clustered network having scale-free degree distribution. When a small ratio of random connections is added ( $\mu=0.1$ ), the error rate increases, achieving its maximum value for $\mu=1.0$. As in the case of the small-world networks, the influence of the model parameter in the error rate is higher at the beginning of training.

In order to analyze the influence of degree-degree correlations, networks with different levels of assortativity were used in the SOM. Figure 13c shows that the error rate tended to be inversely proportional to the assortativity. The same behaviour is obtained for the musical symbols and letters dataset, as shown in Figures $13 \mathrm{f}$ and $13 \mathrm{i}$.

It is also interesting to verify which topological properties tend to be associated with larger error rates. As shown in the previous results, the error rate is more dependent on the topology when a small number of learning steps is used. Therefore, we henceforth consider 30000 learning steps. The results for the digits dataset are shown in Figure 14 and 15. The plots from top to bottom correspond to the small-world, deactivation model and degree-degree correlated network.

Figure 14a shows a plot of the error rate as a function of the average shortest path length, $\langle L\rangle$. Parameter $p$ of the small-world model was used for varying $\langle L\rangle$. The plot shows that the error rate is inversely proportional to $\langle L\rangle$. The variation of the error rate as the clustering coefficient of the network increases is shown in Figure 14b. Since a lattice was considered for the initial configuration of the small-world model, the clustering coefficient is always close to zero. Figure 15a shows the relationship between the error rate and parameter $p$ of the small-world model. The plot indicates that the error rate increases with $p$.

Figures $14 \mathrm{e}, 14 \mathrm{f}$ and $15 \mathrm{c}$ show the relationship between the error rate and, respectively, the average shortest path length, average clustering coefficient and assortativity of the network. Again, the error rate decreases with the average shortest path length. A large decrease in error rate is observed for a slight increase in the clustering coefficient. Also, the error rate is inversely proportional to the assortativity of the network. This is likely caused by the strong relationship between $\langle L\rangle$ and the assortativity, as shown in the inset of Figure 14e. 


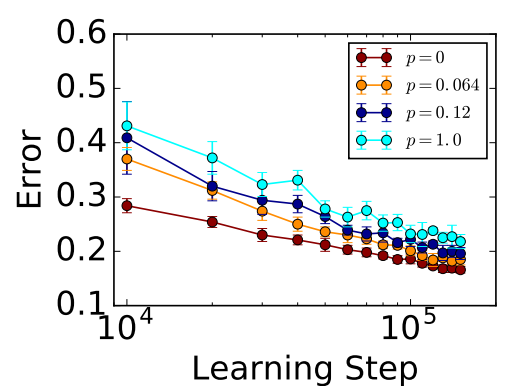

(a) digits dataset

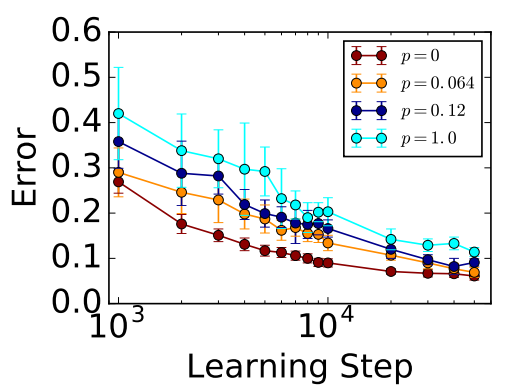

(d) music dataset

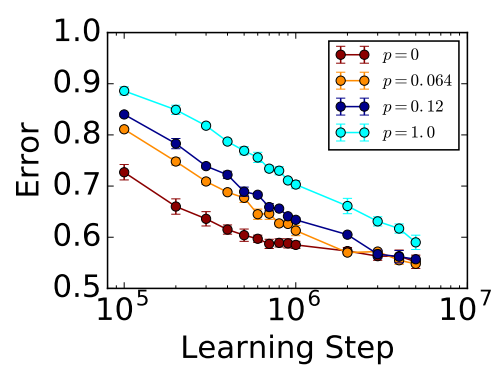

(g) letters dataset

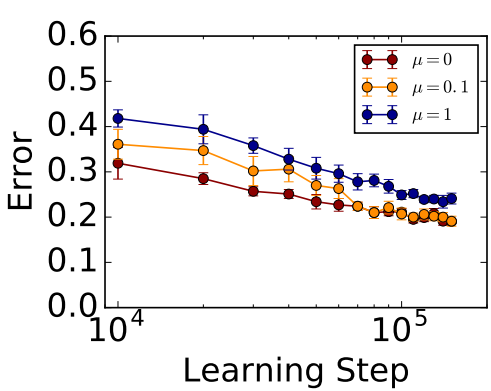

(b) digits dataset

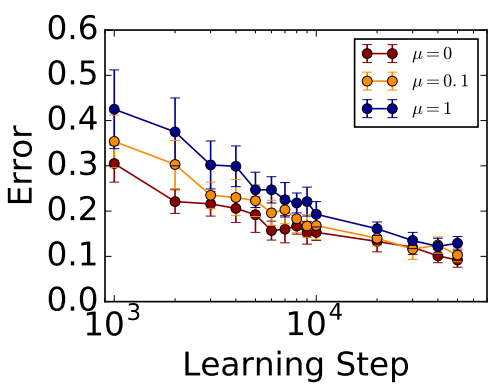

(e) music dataset

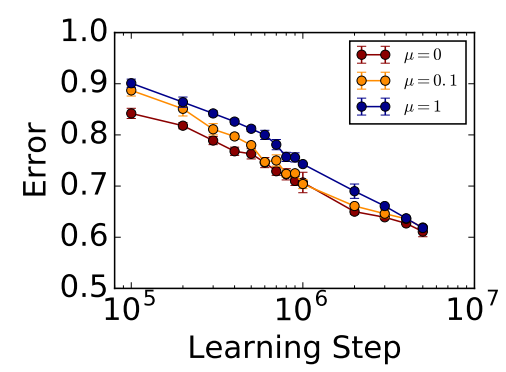

(h) letters dataset

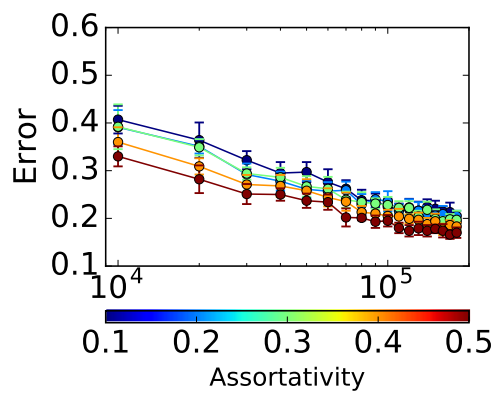

(c) digits dataset

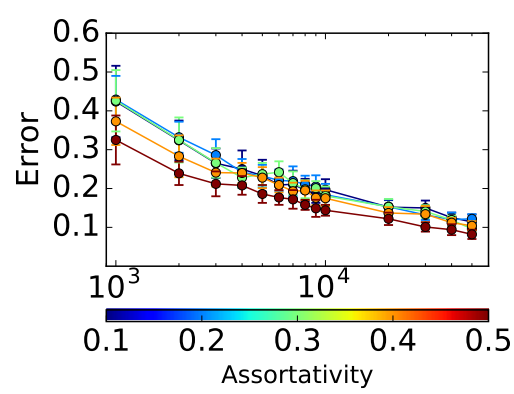

(f) music dataset

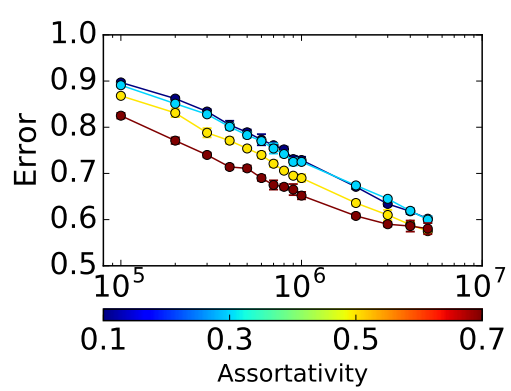

(i) letters dataset

Source: Elaborated by the author.

Figure 13 - Error rate as a function of the number of learning steps for different datasets and network models. The first, second and third rows of plots correspond, respectively, to the digits and musical symbols and letters dataset. Each column correspond to a model network. From left to right correspond to SW, deactivation model and degree correlated networks. The first and second columns correspond to a map size of $50 \times 50$. In the third column a map size of $35 \times 35$ where used. Figures 13a, 13d and 13g show the results for the small-world model. The results for the deactivation model are shown in Figures 13b, 13e and 13h. Figures 13c, $13 \mathrm{f}$ and $13 \mathrm{i}$ show the results for the degree-degree correlated networks. In all cases $\langle k\rangle=4$ was used and the initial learning was set to $\eta=0.08$.

The relationship between the error rate and $\langle L\rangle$ for the deactivation model, shown in Figure $14 \mathrm{c}$, is similar to those observed for the other models. Interestingly, the clustering coefficient seems to have little effect on the error rate (as shown in Figure 14d). Only for large clustering coefficients, on the order of $\langle c c\rangle \approx 0.6$, a decrease in error rate is observed. The plot in Figure 15b indicates that parameter $\mu$ of the deactivation model can have a large influence on the error rate for $\mu<0.2$. It is interesting to note that this parameter has a nonlinear relationship 
with the clustering coefficient of the network, as shown in Figure 15b.

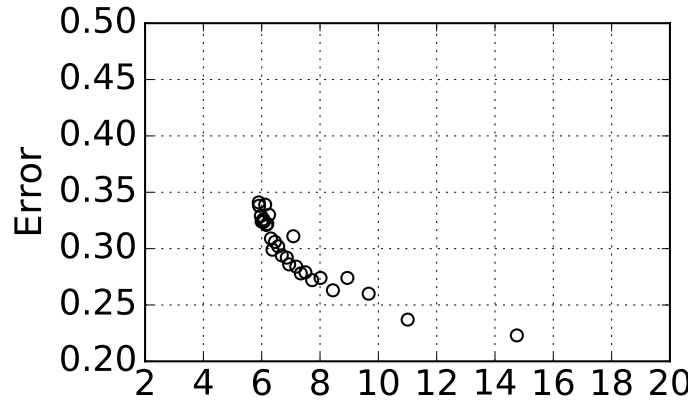

$\langle L\rangle$

(a)

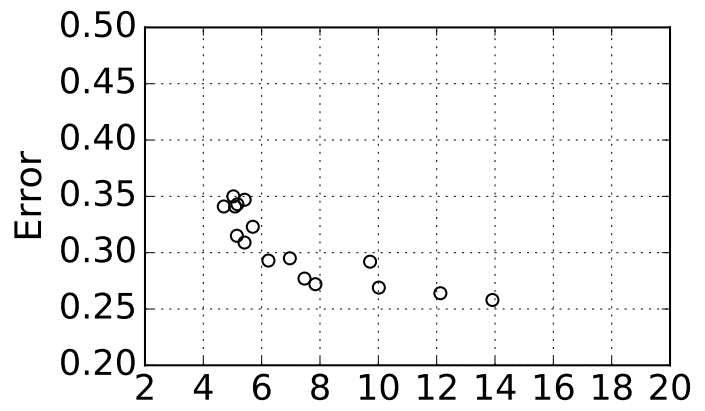

$\langle L\rangle$

(c)

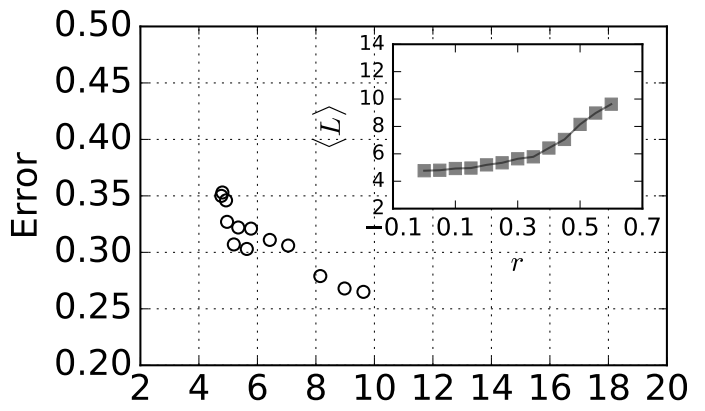

$\langle L\rangle$

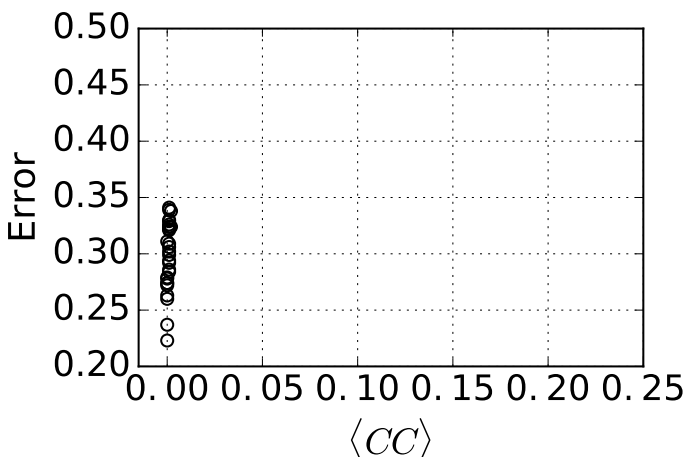

(b)

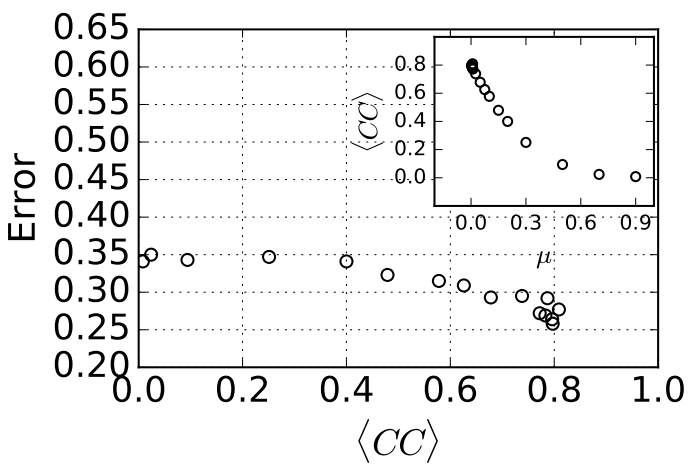

(d)

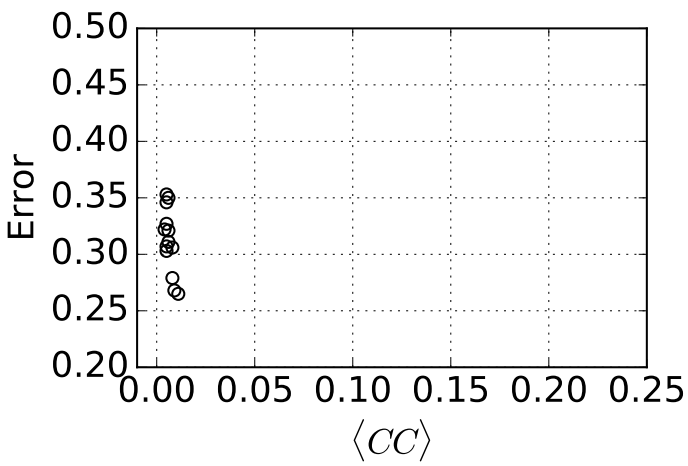

(f)

Source: Elaborated by the author.

Figure 14 - Error rate as a function of network properties for the digits dataset. The plots from top to bottom correspond to the small-world, degree-degree correlated and deactivation models. Inset plots show the relationship between selected pairs of topological measurements.

Was verified how the map size affects the accuracy of each network model. The figures were plot according to the error rate in function of training time as we can see in figure 16. A map size of 20x20, 35x35 and 70x70 was considered in our analysis. In figure 16a we observe that for different sizes of maps there is a progressive and significant decrease in the error rate as the network is trained when the network is regular with $p=0$. On the other hand, when the 


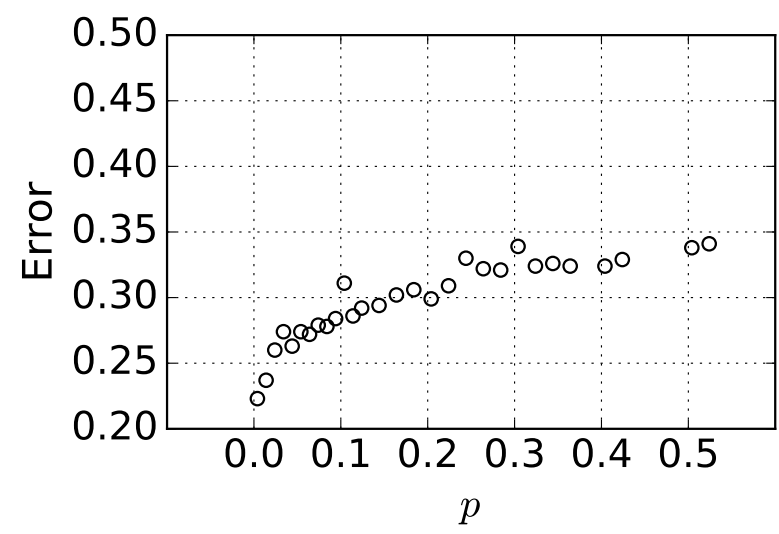

(a) Small world

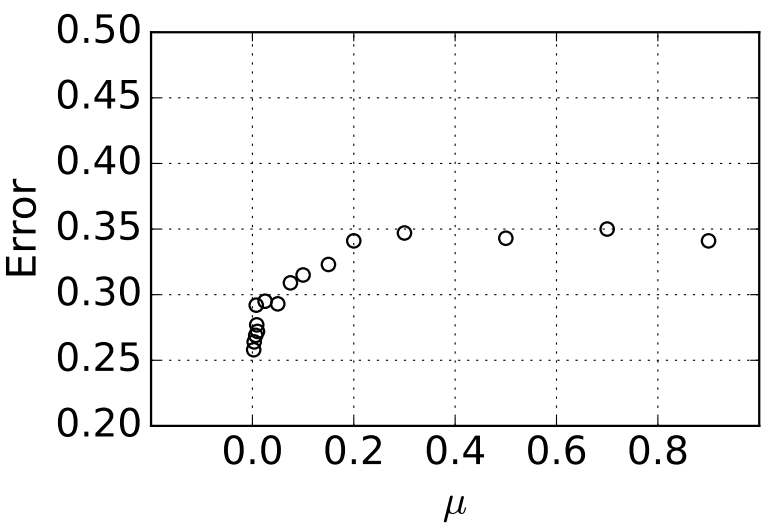

(b) Deactivation model

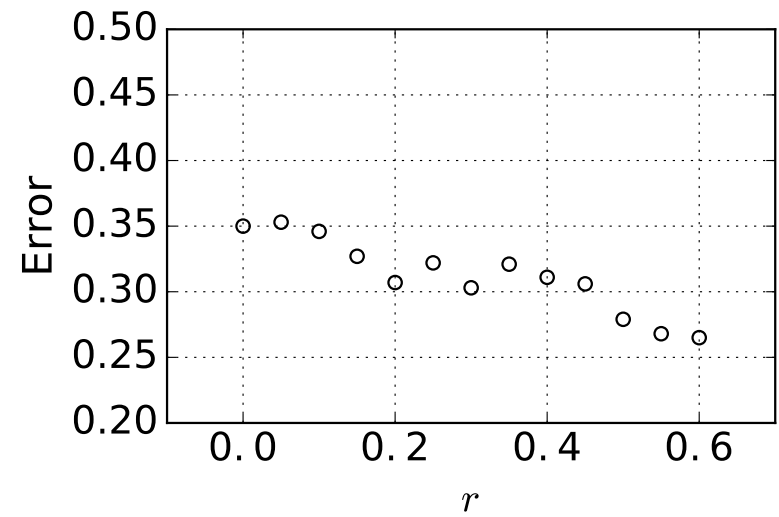

(c) Degree-correlated networks

Source: Elaborated by the author.

Figure 15 - Error rate as a function of model parameters for the digits dataset. The plots from top to bottom correspond to the small-world, degree-degree correlated and deactivation models. 
network becomes disorganized ( $p=1$ ), the error rate varies less significantly as the network size increases. In deactivation model, the lowest error rate is obtained when the network increases the size when $\mu=0$ and the same behaviour is observed with $\mu=0.1$ as we can see in figure 16b. Likewise in degree correlated network when $r=0.5$ and the size of the map is large the error decrease more quickly at short and middle training than when the network is smaller as can be appreciated in figure 16c.

\subsection{Analysis of topological properties of map SOM}

The Self-organization exhibited by topological maps is given by a spontaneous pattern formation as a result of the interactions of components whose nature is non-linear (KELSO; OPSTAL, 1996). In the human brain, the organization is distributed over a network of interacting neurons where different brain regions are specialized for different tasks (HEYLIGHEN et al., 2001). A process of self-organization in artificial neural networks as observe too, which was inspired by the process of self-organization exhibited by topological maps in the cortex. In SOM network one requirement for the organization is obtained between a competition process between neurons and another requirement is through an update of weights only in the local vicinity of the winner neuron.

In order to quantify the topological properties of topological map SOM, we consider the following measurements. The average number of elements per group $\bar{e}_{\text {groups }}$, the average number of groups $\bar{n}_{\text {group }}$ and the size of the largest group $S_{\text {group }}$ by map. In the same way, we calculate the standard deviation of the average number of elements $\bar{\sigma}_{e_{\text {groups }}}$, the modularity $Q$. Maps of size $35 \times 35, \sigma=15$ with $\langle k\rangle=4$ were used in the experiments. For each model, we consider 10 realizations using the digits dataset.

In Figure 17 we can see an example of the output of topological map with a map size of 10x10 and organized by subsets of neurons under the same label. The groups or modules were formed by the similarity of the input patterns. In the example of the figure below the map contains eight groups that represent digits from 0 to 7 . The digit 2 is separated into two groups and the digits 7 and 5 form groups of a single element.

For the output map indicated as an example we calculate the topological measurements of the map: $\bar{e}_{\text {groups }}=11, \bar{n}_{\text {groups }}=9$ and $S_{\text {group }}=32$.

A more systematic investigation of the SOM is shown in Figure 18 containing the results for the topological measures as a function of error. Each point represents a different number of learning steps used in the training (in the interval $\left[10^{3}, 10^{6}\right]$ ). It was considered a small-world network, deactivation model and correlated degree network. The plots from left to right row correspond to the average number of elements per group, the average number of groups and the size of the largest group, respectively. For small-world networks with $p=0$ the error rate is positively correlated with the number of identified groups and thus negatively correlated with 

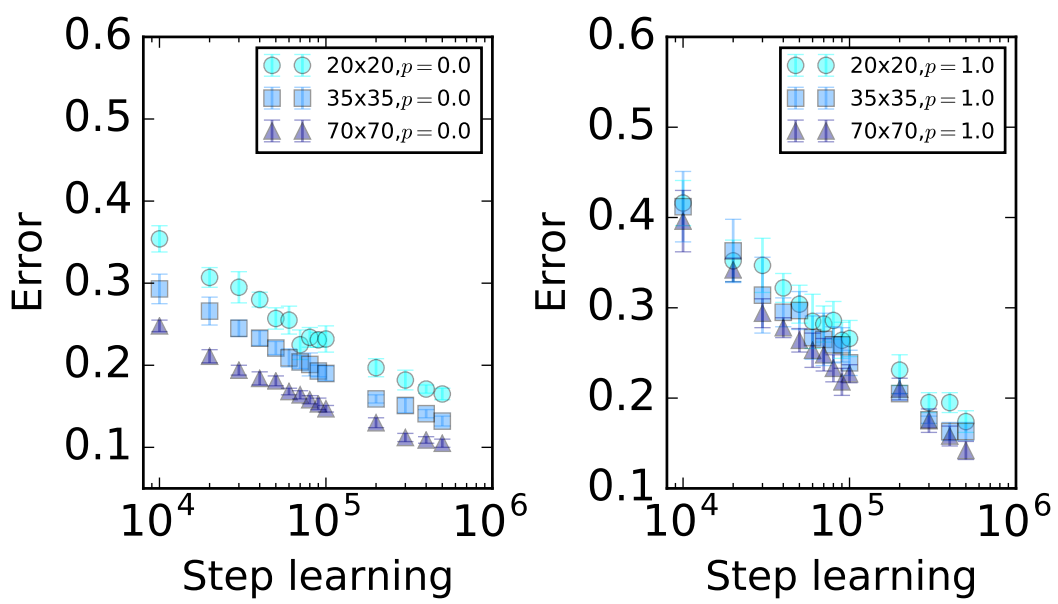

(a)
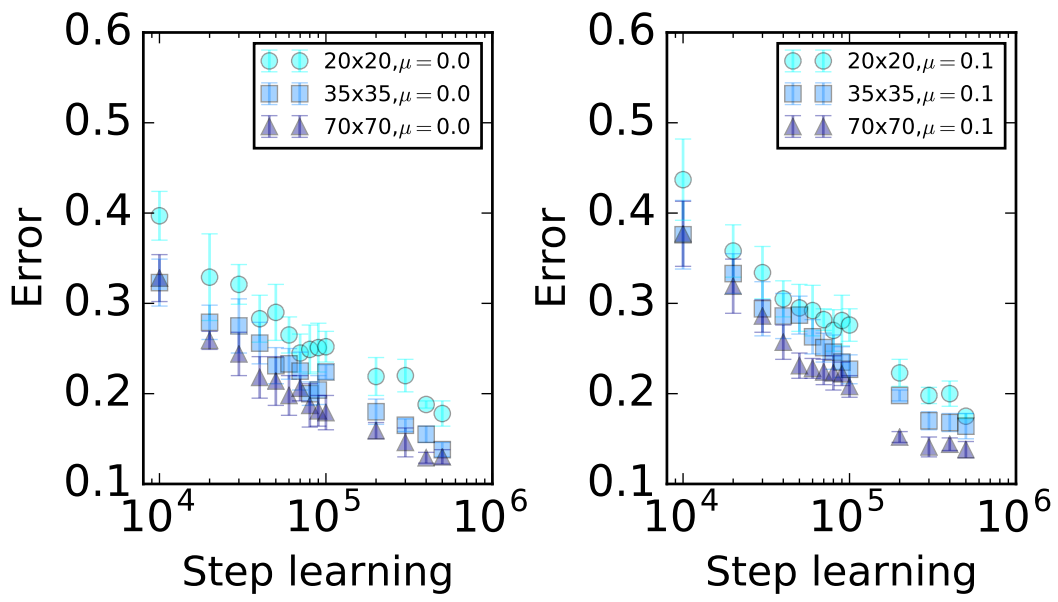

(b)
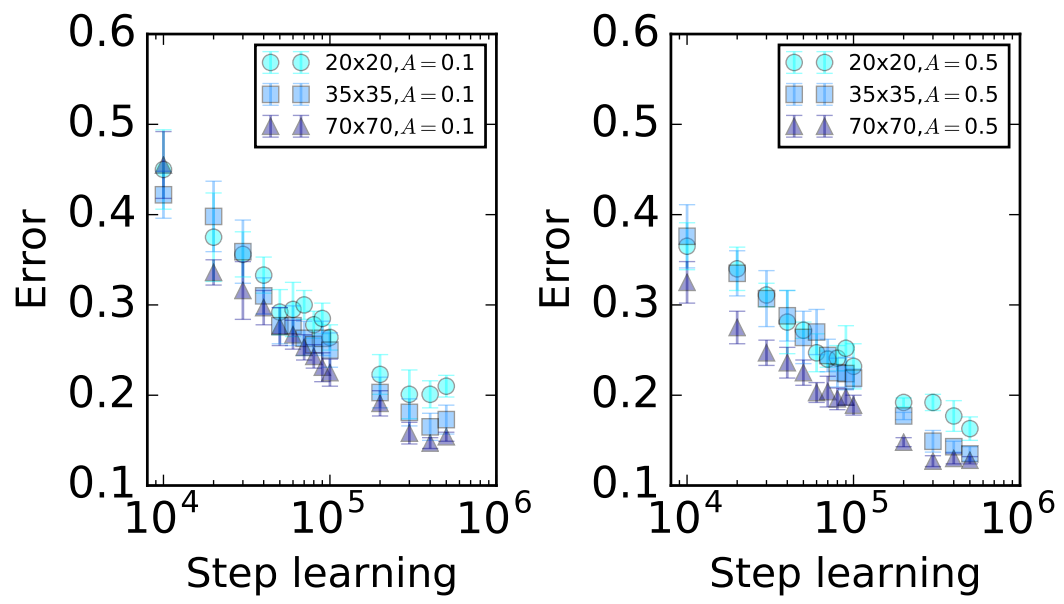

(c)

Source: Elaborated by the author.

Figure 16 - Error rate of the SOM maps as a function of training time. From top to bottom the plots correspond to small-world, deactivation and assortative networks. Map sizes of 20x20, 35x35 and $70 \times 70$ were used. 


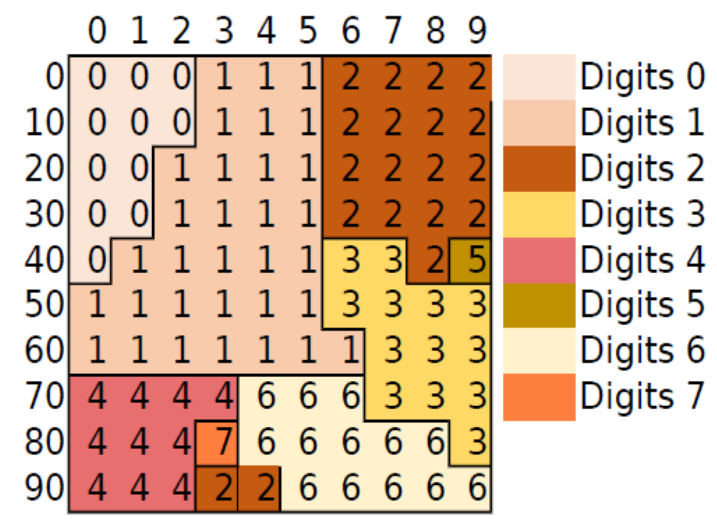

Source: Elaborated by the author.

Figure 17 - Example of SOM result for a map of size 10x10.

the average group size (Figures 18a and 18b). On the other hand, for $p>0$ the error rate is not influenced by the number of groups and the average group size. The deactivation model follows a similar behavior of the small-world model. The number of elements per group increases and the number of groups decreases for lower error rate(Figures $18 \mathrm{c}$ and $18 \mathrm{~d}$ whn $\mu=0$, while only small variations on the groups properties are observed for $\mu=0.1$. An inverse behavior is observed for assortative networks with $r=0.5$ (Figures 18e and 18f). IN this case, having more groups lead to lower error rates. This indicates that the network os being fragmented into small groups containing similar patterns. A different scenario happens with $r=0.1$, where the mean number elements per group remains constant. In Figure 19 whe show the size of the largest group for the sames cases reported in Figure 18. The variations of the size of the largest group seems negligible on the majority of cases, the notable exception being assortative networks with $r=0.5$, where the largest group has a sharp decrease in size for lower error rates.

In figure 20 we can observe the standard deviation of the mean numbers elements per group in function of error rate. Each symbol corresponds to the standard deviation for different learning step. The standard deviation in a small-world network when $p=0$ approaches zero when the training time increases indicating that the mean number elements per group tend to be close to the mean as we can see in figure 20a. For other values of $p$ the standard deviation is slightly above the mean. At short training, a high data dispersion is observed when $r=0.5$, but as the training time increases the dispersion of data gradually decreases, as can be seen in figure 20c.

In order to quantify how well the neurons in the SOM networks can be partitioned into communities, the modularity measure was calculated. The communities corresponds to the membership of each neuron obtained from the output of SOM map after training steps. Note 


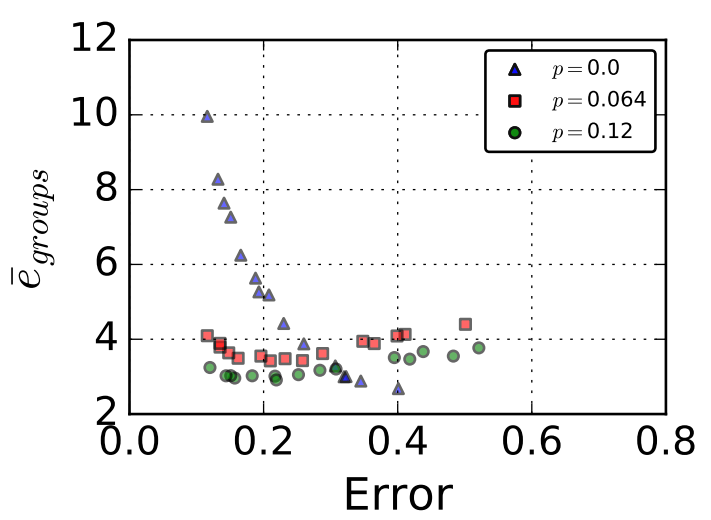

(a)

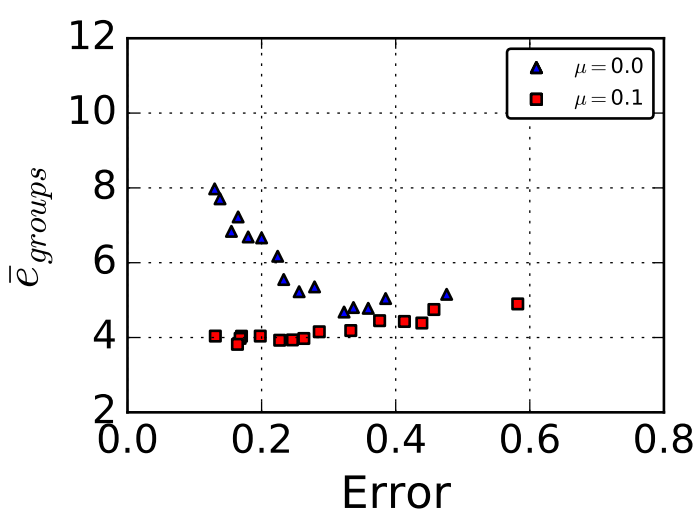

(c)

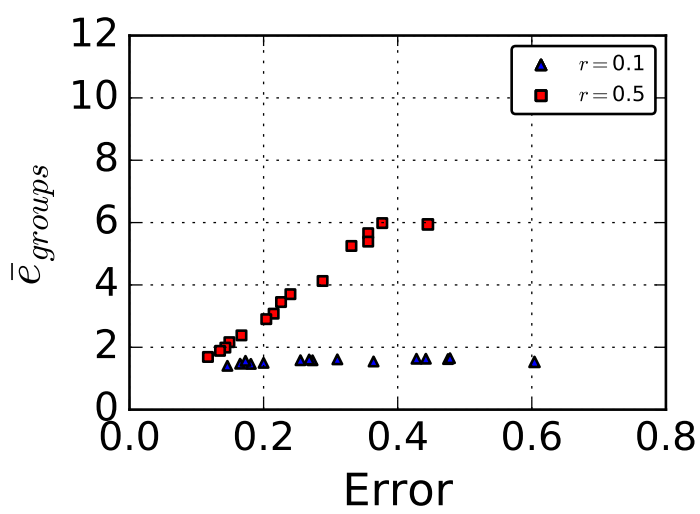

(e)

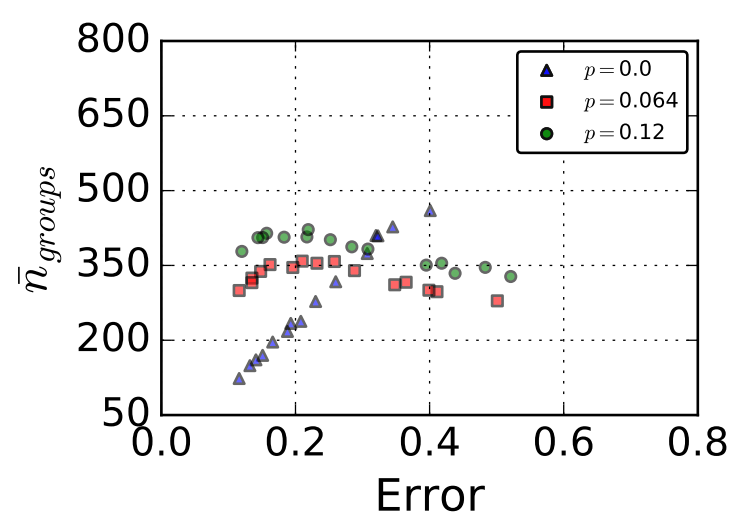

(b)

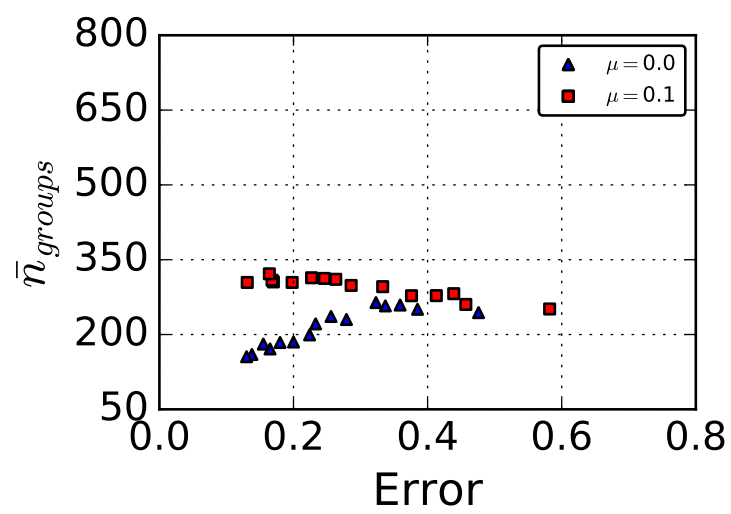

(d)

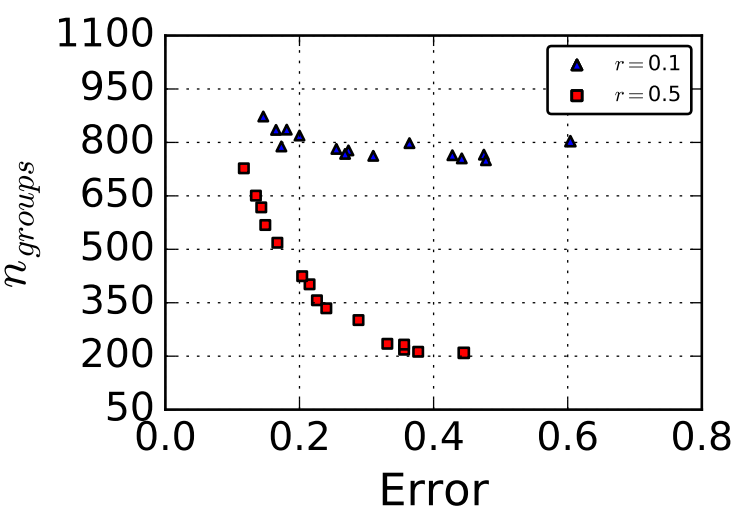

(f)

Source: Elaborated by the author.

Figure 18 - Average number of neurons per group, $\bar{e}_{\text {groups }}$, and number of groups, $\bar{n}_{\text {groups }}$, as a function of error rates obtained for distinct number of learning steps used when training SOM models. The plots from top to bottom correspond to small-world, deactivation and assortative networks. 


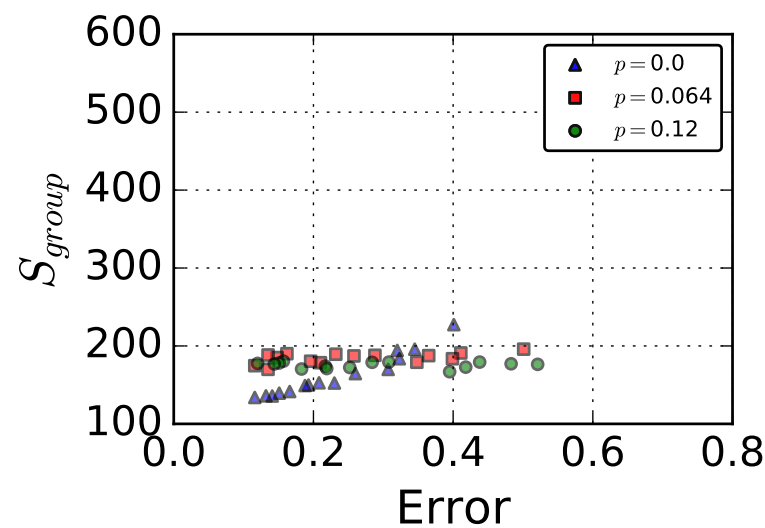

(a)

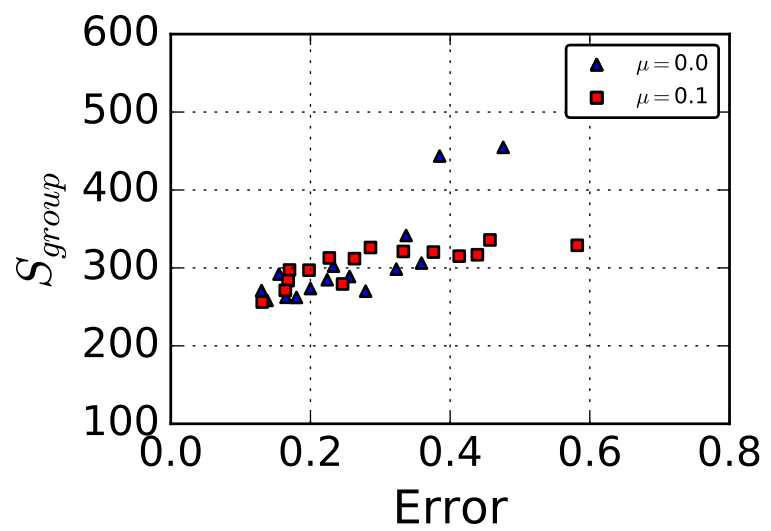

(b)

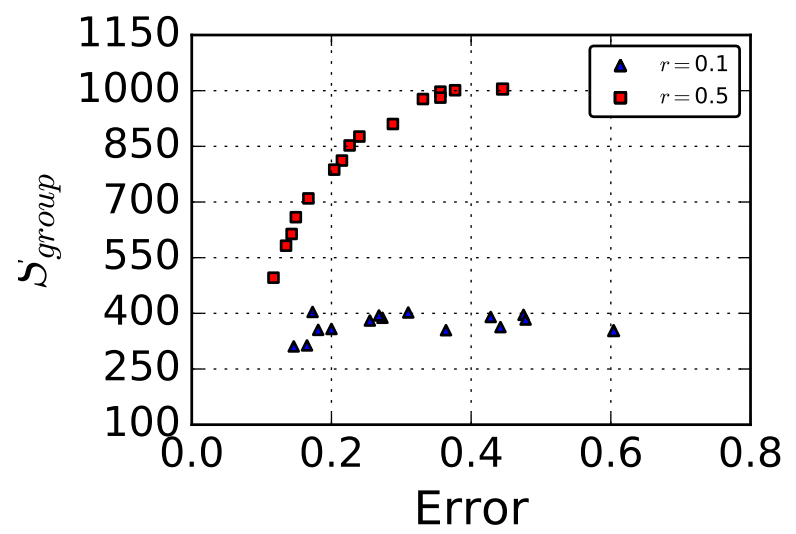

(c)

Source: Elaborated by the author.

Figure 19 - Size of the largest group as a function of the error rate for different network models. The models are (a) small-world, (b) deactivation and (c) assortative. 

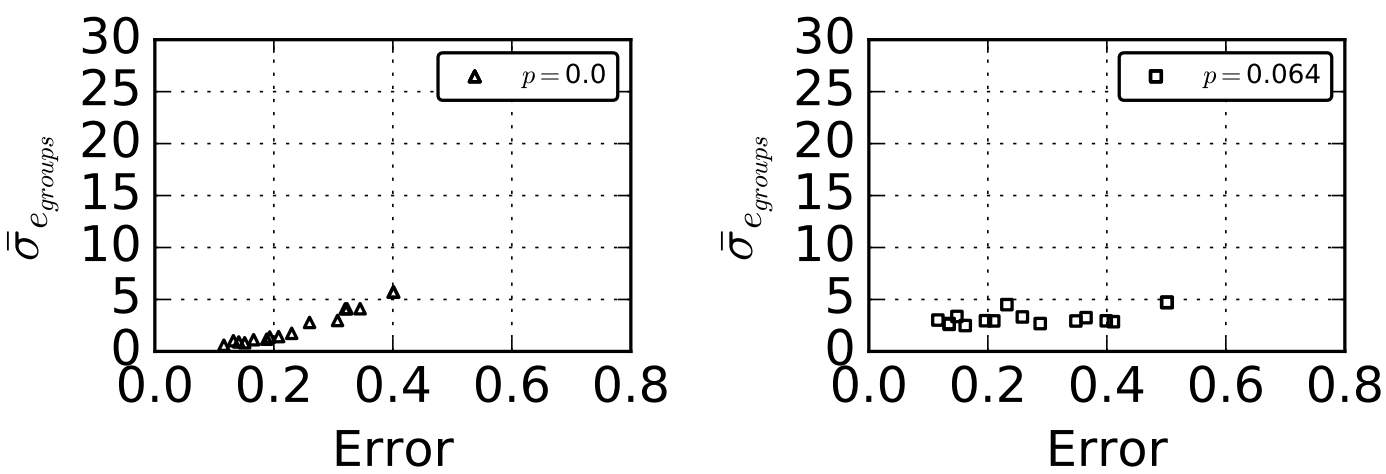

(a)
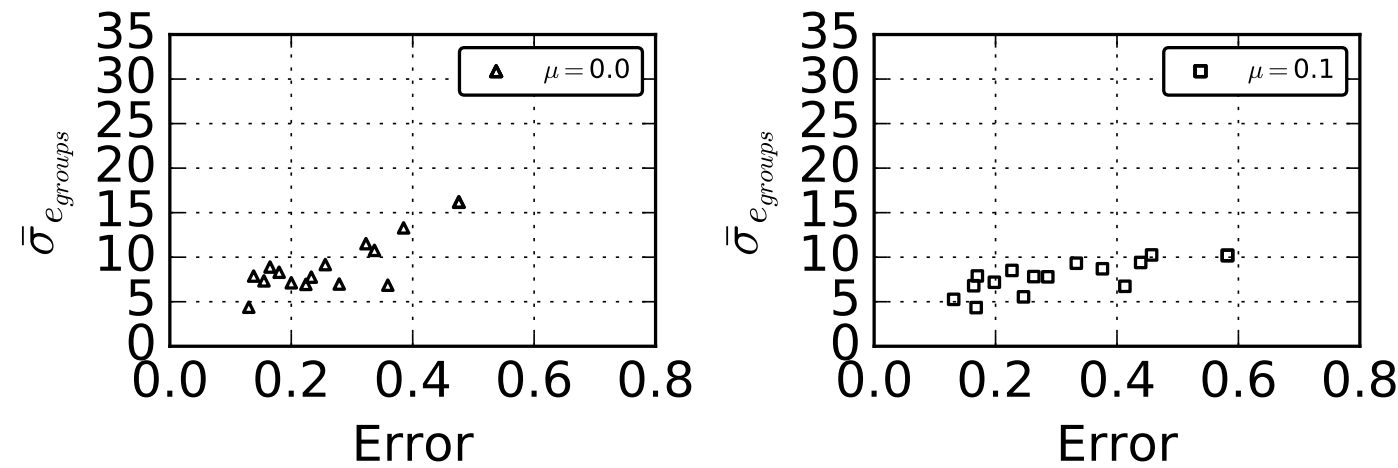

(b)
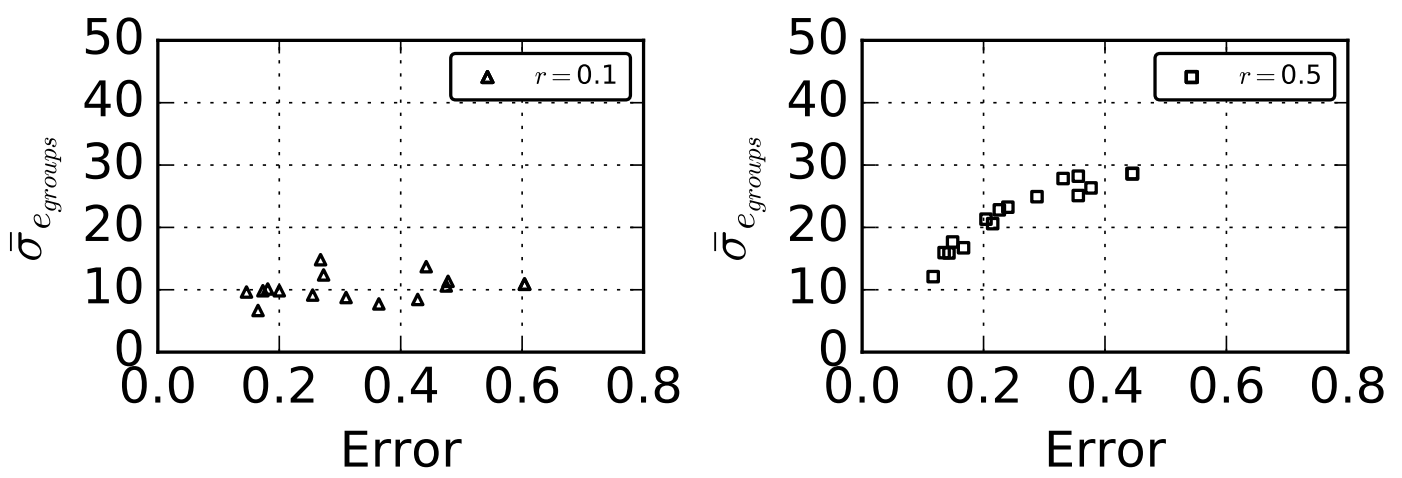

(c)

Source: Elaborated by the author.

Figure 20 - Standard deviation of group sizes as a function of the error rate. The plots from top to bottom correspond to small-world, deactivation and assortative networks. 
that the maximum value of modularity is 1 . A high modularity suggests a clear community structure. High modularity and a low number of modules imply efficient functional segregation and integration. In brain networks, segregation and integration are both necessary for brain functions (HILGETAG et al., 2000). Figure 21 shows the modularity, $Q$ as a function of the number of learning steps. Inset inside each plot show the modularity as a function of the error rate. From top to bottom the plots correspond to small world, deactivation and assortative networks. The modularity for the small-world model with $p=0$, shown in Figure $21 \mathrm{~b}$, increases with the training time. going from 0.2 to approximately 0.65 . For $p>0$, the modularity does not significantly change during training. A similar behavior is observed in the deactivation model, wich is shown in Figure 21c. Similarly to the small-world and deactivation model, the results for the assortative model show significant differences according to the parameter used to generate the network. For low assortativity, the modularity stays at around 0.3 during the whole training procedure, as shown in Figure 21e. On the other hand, large assortativity leads to an increase in modularity as the number os learning steps increases. This indicates that there are well-defined groups within the map, although we know that the groups are composed of few elements, as observed in the previous results for this model. All in all the results indicate that higher modularity leads to lower error rates, and that topologies having less shortcuts tend to promote higher modularity.

\subsection{Final remarks}

In this chapter was investigated if different networks topologies yield significant differences with respect to performance. Analyzing the performance of SOM neural networks with different topologies such as small world, correlated networks and deactivation model of Klemm. We found that the interconnecting topology has an influence on the performance of the networks at short and middle learning time scales. Depending on the type of database learning can take from medium to long times. In addition, the effect of assortativity on the efficiency of interconnected networks was investigated. The study also revealed that excessive assortativity led to improving network performance compared to a low assortativity network due to the creation of small clusters in the network, preventing the update process to be expanded to the other modules. It has also been shown that depending on the network topology it is possible to observe differences in accuracy when considering different map size.

To measure the modular structure of SOM network was used measures such as the average number of elements by group, the number of group, the largest group, the modularity measure and the edge distributions of edges inter subgraph. A self-organization map with a small world topology with $p=0$ organize the patterns into dense subgraphs. Also there is a visible growth of the modularity as the step learning increases, whose value varies from 0.4 to approximately 0.65 . On the other hand, adding shortcuts with $p>0$ was shown that there is a deterioration of organization. The network does not seem to self-organize as the training time. 


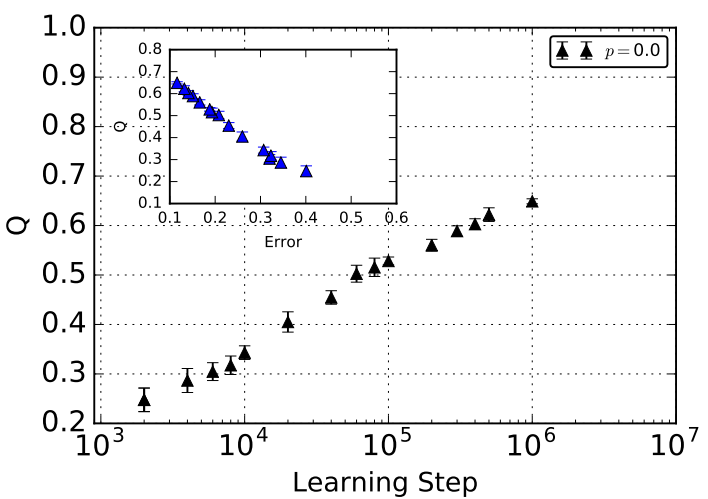

(a)

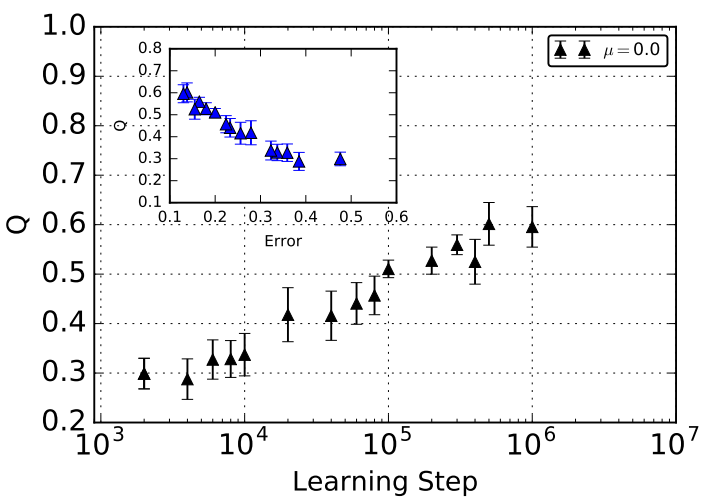

(c)

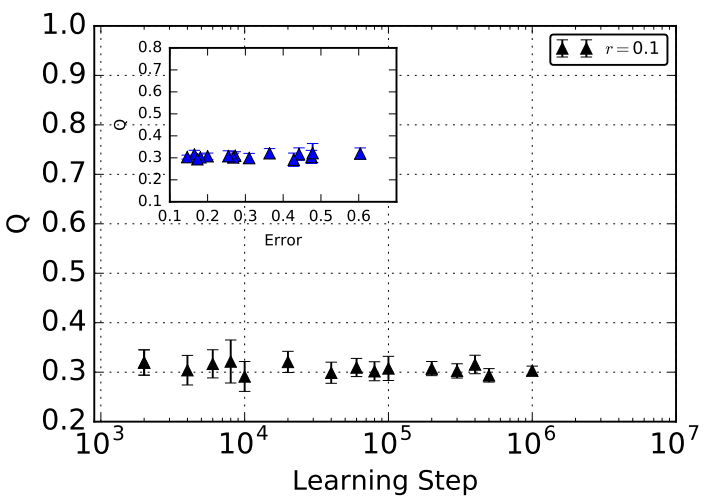

(e)

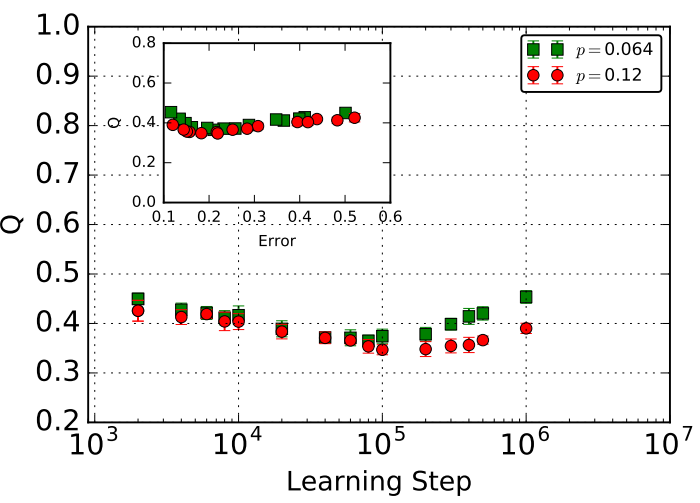

(b)

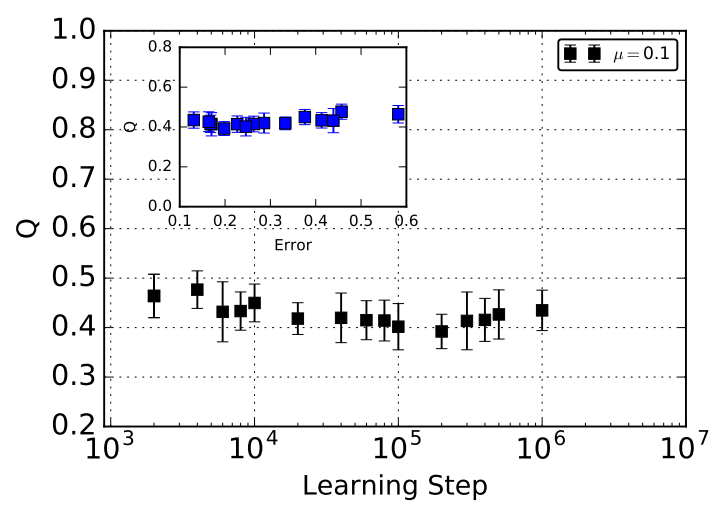

(d)

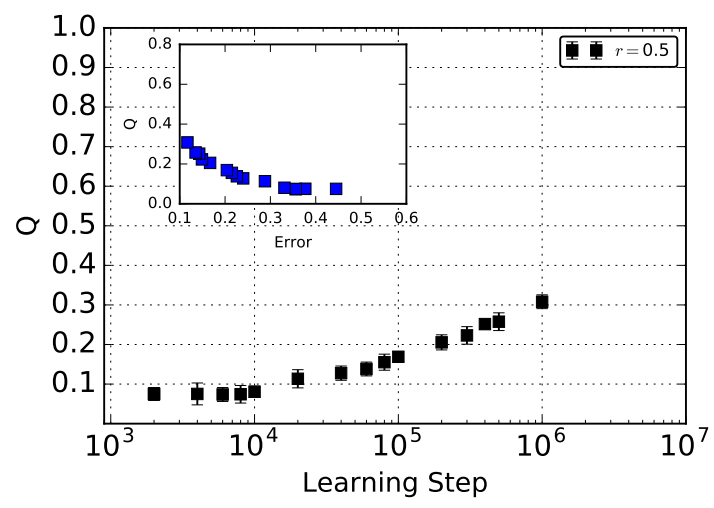

(f)

Source: Elaborated by the author.

Figure 21 - Modularity as a function of training time for the digits dataset. From top to bottom correspond to small world network, deactivation model and degree correlated networks. Inset plots show the relationship between modularity and error rate. 
The same behavior is obtained with deactivation model. With $\mu=0.1$ in the deactivation model tends to slightly decrease the average number of elements by group and the increase the number of group with increased training.

In assortative networks with a high level of assortativity, the tendency is that the map to be organized in many groups of few elements, favoring the increase of the connectivity between modules than within modules. 
CHAPTER

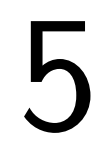

(2)

\section{CONCLUSIONS}

The great potential of Self-organization map as a tool for the analysis of data is demonstrated by the innumerable papers that has been published and by the amount of applications that the network is used. Our main objective in this thesis was to study the kohonen network from two perspectives. From the perspective of complex networks where: (i) properly quantify the impact of topology in performance of SOM network (Chapter4), (ii) quantify the topological properties of topographic output map (Chapter4) and from the perspective of machine learning where: (i) throught of a large diversity of heterogeneous datasets with defined properties, was quantified the performance of SOM as a method of clustering algorithm (Chapter3), (ii) and was developed a comparison of the performance of SOM and other popular clustering methods (Chapter3).

We began chapter 3, indicating some topological features that real network networks exhibit in comparison to the regular network and the random model. We point out that has been proposed models capable of capturing some specific characteristics of real networks. In order to study a specific characteristic, as is the case of assortativity. Different proposed models vary the level of assortativity without altering other characteristics of the network. Generating networks with different correlated degress. We found interesting to show how such a variation of assortativitie can affect the performance of the SOM network.

Another model that was considered was the SW model because it has some characteristics that we find in real networks and also because through a process of rewiring the model interpolates between a regular structure and a random one. The initial step to began our analysis of the topological influence of the network through simulations, was evaluate the error rate of network SOM considering the network models that were mentioned previously and three different dataset such as: letters, musical symbols and digits. Was observed that the interconnecting topology has an influence on the performance of the networks at short and middle learning time scales. After a long learning period, the differences in error rate between distinct network models seem to disappear. Depending on the type of database learning can take from medium to long times as indicated in the figure 13. It has been shown that different levels of assortativity in the network 
influence the accuracy of SOM. A high assortativity implies the creation of small clusters in the network, preventing the update process to be expanded to the other modules of the network, resulting in a decrease in the error rate as indicated in the figure $13 \mathrm{c}$.

In addition, we analyzed the influence of topological measurements such as the average of clustering coefficient $(\langle C C\rangle)$ and the average path length $(\langle L\rangle)$ as functions of the error rates obtained for these models. In the deactivation model, adding a small amount of long-range connections $(10 \%)$ tended to worsen the network performance.

In order to study the topological properties of the output map created by SOM network we consider the following measures: The average number of elements per group $\left(\bar{e}_{\text {groups }}\right)$, average number of groups $\left(\bar{n}_{\text {group }}\right)$, size of the largest group $\left(S_{\text {group }}\right)$ by map and the modularity $(Q)$ that quantifies how dense are the groups formed between communities of groups and within each community. The topological properties were calculated for different training times of the network in order to observe the topographic changes of the map as the training time increases.

First, through the results we can see how graph theoretical techniques help to understand the influence of the topology on acuracy and the self-organization of the SOM network. In small world network when the parameter of rewiring $p=0$ the mean number of elements per group increases as the time increase. This means that the groups begin to be denser and more defined, which is also corroborated with the measure of modularity whose value is around 0.7. However the $p>0.064$ and $p=0.12$ the number of elements per group remains the same as training time increases, and in turn, the network does not self-organize as time increases. The modularity of the network is around 0.45 . The same behavior happens with the deactivation model. When $\mu=0$ the behavior is similar to a small world network with $p=0$. A small amount of addition of long-range connections with $\mu=0.1$ reduces the modularity from 0.6 to 0.45 approximately. At short time scale, when the assortativity has high values the modularity obtained is around of 0.1 , which indicates that the network is disorganized. At middle short time scale the network begins to self-organize in small groups, obtaining a modularity of 0.3 aproximately.

In this work we also study the sensitivity of the clustering methods with regard to their parameters configuration. Considering a configuration of parameters by default we show that the spectral algorithm showed the best performance. On the other hand the kohonen network had an average performance. We also found that the default configuration of the adopted implementations was not accurate. In these cases, a simple approach based on random selection of parameters values proved to be a good alternative to improve the performance.

When considering a two-class problem (DB2C2F), a small improvement was observed for the SOM algorithm through the variation of radius. With respect to the multidimensional analysis for datasets containing ten classes and two features, the performance of the algorithms for the multidimensional selection of parameters was similar to that using the default parameters. In random configuration of parameters we also observed that the SOM algorithm tends to improve its acuracy when the number of classes is low. 
In summary, we consider that our main contributions on the topic are as follows:

- Comprehensive methodology for generating a large diversity of heterogeneous datasets with precisely defined properties.

- Analysis of the influence of input parameters configuration in function of accuracy over the network Self-organization map and other algorithms of clustering.

- Verification of topological influences on accuracy rate in a self-organization map network.

- Quantification of the topological properties of output map SOM after training step, considering different topologies.

The reported findings pave the way to several further investigations related to topological influence on SOM, such as studying the effect of the interconnection topology on the robustness of the networks, treat the neurons in terms of groups associated to each learn pattern, and to consider other learning dynamics. 

ABBAS, O. A. et al. Comparisons between data clustering algorithms. Int. Arab J. Inf. Technol., v. 5 , n. 3, p. 320-325, 2008. Citation on page 47.

AGGARWAL, C. C.; REDDY, C. K. Data Clustering: Algorithms and Applications. 1st. ed. -: Chapman \& Hall/CRC, 2013. 652 p. ISBN 1466558210, 9781466558212. Citations on pages 45 and 46.

AGHAJARI, E.; CHANDRASHEKHAR, G. D. Self-organizing map based extended fuzzy c-means (seefc) algorithm for image segmentation. Applied Soft Computing, Elsevier, v. 54, p. 347-363, 2017. Citation on page 38.

AHMAD, A.; YUSOF, R. A modified kohonen self-organizing map (ksom) clustering for four categorical data. Jurnal Teknologi, Penerbit Universiti Teknologi Malaysia, v. 78, n. 6-13, p. 75-80, 2016. Citation on page 27.

ALAHAKOON, D.; HALGAMUGE, S. K.; SRINIVASAN, B. Dynamic self-organizing maps with controlled growth for knowledge discovery. IEEE Transactions on neural networks, IEEE, v. 11, n. 3, p. 601-614, 2000. Citation on page 38.

ALDECOA, R.; MARÍN, I. Surprise maximization reveals the community structure of complex networks. Scientific reports, Nature Publishing Group, v. 3, p. 1060, 2013. Citation on page 33.

AMANCIO, D. R.; COMIN, C. H.; CASANOVA, D.; TRAVIESO, G.; BRUNO, O. M.; RODRIGUES, F. A.; COSTA, L. da F. A systematic comparison of supervised classifiers. PloS one, Public Library of Science, v. 9, n. 4, p. e94137, 2014. Citation on page 52.

ANKERST, M.; BREUNIG, M. M.; KRIEGEL, H.-P.; SANDER, J. Optics: Ordering points to identify the clustering structure. SIGMOD Rec., ACM, New York, NY, USA, v. 28, n. 2, p. 49-60, jun 1999. ISSN 0163-5808. Available: <http://doi.acm.org/10.1145/304181.304187>. Citation on page 45 .

. Optics: Ordering points to identify the clustering structure. In: DELIS, A.; FALOUTSOS, C.; GHANDEHARIZADEH, S. (Ed.). SIGMOD 1999, Proceedings ACM SIGMOD International Conference on Management of Data, June 1-3, 1999, Philadephia, Pennsylvania, USA. [S.1.]: ACM Press, 1999. p. 49-60. ISBN 1-58113-084-8. Citation on page 45.

ARBIB, M. A. The handbook of brain theory and neural networks. [S.1.]: MIT press, 2003. Citations on pages 25 and 39.

ARRUDA, G. F. de; COZZO, E.; MORENO, Y.; RODRIGUES, F. A. On degree-degree correlations in multilayer networks. Physica D: Nonlinear Phenomena, Elsevier, v. 323, p. 5-11, 2016. Citations on pages 29 and 37.

BACKER, E. Computer-assisted reasoning in cluster analysis. [S.1.]: Prentice Hall International (UK) Ltd., 1995. Citation on page 41. 
BARABASI, A.-L.; ALBERT, R. Emergence of scaling in random networks. Science, v. 286, n. 5439, p. 509-512, 1999. Citation on page 35.

BARRAT, A.; BARTHELEMY, M.; VESPIGNANI, A. Dynamical processes on complex networks. [S.1.]: Cambridge university press, 2008. Citation on page 71.

BASHEER, I. A.; HAJMEER, M. Artificial neural networks: fundamentals, computing, design, and application. Journal of microbiological methods, Elsevier, v. 43, n. 1, p. 3-31, 2000. Citation on page 25.

BASSETT, D. S.; BULLMORE, E. Small-world brain networks. The neuroscientist, Sage Publications Sage CA: Thousand Oaks, CA, v. 12, n. 6, p. 512-523, 2006. Citation on page 34.

BERGÉ, L.; BOUVEYRON, C.; GIRARD, S. HDclassif: an R Package for Model-Based Clustering and Discriminant Analysis of High-Dimensional Data. Journal of Statistical Software, University of California, Los Angeles, v. 46, n. 6, p. 1-29, 2012. Available: <https: //hal.archives-ouvertes.fr/hal-00541203>. Citations on pages 51 and 58.

BISHOP, C. et al. Pattern recognition and machine learning. [S.1.]: Springer-Verlag New York, 2006. Citations on pages 27 and 46.

BISHOP, C. M. Neural networks for pattern recognition. [S.1.]: Oxford university press, 1995. Citation on page 25.

BOCCALETTI, S.; LATORA, V.; MORENO, Y.; CHAVEZ, M.; HWANG, D.-U. Complex networks: Structure and dynamics. Physics Reports, Elsevier, v. 424, n. 4, p. 175-308, 2006. Citation on page 33.

BROHÉE, S.; HELDEN, J. van. Evaluation of clustering algorithms for protein-protein interaction networks. BMC Bioinformatics, v. 7, n. 1, p. 1-19, 2006. ISSN 1471-2105. Available: $<$ http://dx.doi.org/10.1186/1471-2105-7-488>. Citations on pages 47, 48, and 49.

BULLMORE, E.; SPORNS, O. The economy of brain network organization. Nature Reviews Neuroscience, v. 13, n. 5, p. 336-349, 2012. Citation on page 36.

BURDICK, D.; CALIMLIM, M.; GEHRKE, J. Mafia: A maximal frequent itemset algorithm for transactional databases. In: Proceedings of the 17th International Conference on Data Engineering. Washington, DC, USA: IEEE Computer Society, 2001. p. 443-452. ISBN 0-76951001-9. Citation on page 50.

BURGUILLO, J. C. Using self-organizing maps with complex network topologies and coalitions for time series prediction. Soft Computing, Springer, v. 18, n. 4, p. 695-705, 2014. Citation on page 27.

CARIDAKIS, G.; KARPOUZIS, K.; DROSOPOULOS, A.; KOLLIAS, S. Somm: Self organizing markov map for gesture recognition. Pattern Recognition Letters, Elsevier, v. 31, n. 1, p. 52-59, 2010. Citation on page 25.

CLAUSET, A.; NEWMAN, M. E.; MOORE, C. Finding community structure in very large networks. Physical review E, APS, v. 70, n. 6, p. 066111, 2004. Citation on page 33.

COHEN, G.; AFSHAR, S.; TAPSON, J.; SCHAIK, A. van. Emnist: an extension of mnist to handwritten letters. arXiv preprint arXiv:1702.05373, 2017. Citation on page 72. 
CORRIDONI, J. M.; BIMBO, A. D.; LANDI, L. 3d object classification using multi-object kohonen networks. Pattern Recognition, Elsevier, v. 29, n. 6, p. 919-935, 1996. Citations on pages 25 and 39 .

COSTA, I. G.; CARVAlHO, F. d. A. T. d.; SOUTO, M. A.-1. C. P. d. Comparative analysis of clustering methods for gene expression time course data. Genetics and Molecular Biology, scielo, v. 27, p. 623 - 631, 00 2004. ISSN 1415-4757. Available: <http://www.scielo.br/scielo. php?script=sci_arttext\&pid=S1415-47572004000400025\&nrm=iso $>$. Citations on pages 47, 49 , and 50.

COSTA, L. d. F.; JR, O. N. O.; TRAVIESO, G.; RODRIGUES, F. A.; BOAS, P. R. V.; ANTIQUEIRA, L.; VIANA, M. P.; ROCHA, L. E. C. Analyzing and modeling real-world phenomena with complex networks: a survey of applications. Advances in Physics, Taylor \& Francis, v. 60, n. 3, p. 329-412, 2011. Citation on page 26.

COVER, T.; THOMAS, J. Elements of Information Theory. [S.l.: s.n.], 2012. 792 p. ISBN 9781118585771. Citation on page 43.

DEMPSTER, A.; LAIRD, N.; RUBIN, D. Maximum likelihood from incomplete data via the em algorithm. Journal of the Royal Statistical Society. Series B (Methodological), Royal Statistical Society, v. 39, n. 6, 1977. Citation on page 45.

DITTENBACH, M.; RAUBER, A.; MERKL, D. Uncovering hierarchical structure in data using the growing hierarchical self-organizing map. Neurocomputing, Elsevier, v. 48, n. 1-4, p. 199-216, 2002. Citation on page 38.

DOUGHERTY, E. R.; BARRERA, J.; BRUN, M.; KIM, S.; CESAR, R. M.; CHEN, Y.; BITTNER, M.; TRENT, J. M. Inference from clustering with application to gene-expression microarrays. Journal of Computational Biology, Mary Ann Liebert, Inc., v. 9, n. 1, p. 105-126, 2002. Citations on pages 47 and 48.

DUDA, R.; HART, P.; STORK, D. Pattern classification. [S.1.]: John Wiley \& Sons, 2012. Citation on page 46.

DUNN, J. C. A fuzzy relative of the isodata process and its use in detecting compact well separated clusters. Cybernetics, Taylor and Francis, v. 3, p. 32-57, 1973. Citation on page 44.

ERDŐS, P.; RÉNYI, A. On the evolution of random graphs. Citation on page 34.

ERKAYMAZ, O.; ÖZER, M.; YUMUŞAK, N. Impact of small-world topology on the performance of a feed-forward artificial neural network based on 2 different real-life problems. Turkish Journal of Electrical Engineering \& Computer Sciences, The Scientific and Technological Research Council of Turkey, v. 22, n. 3, p. 708-718, 2014. Citation on page 26.

ERMAN, J.; ARLITT, M.; MAHANTI, A. Traffic classification using clustering algorithms. In: ACM. Proceedings of the 2006 SIGCOMM workshop on Mining network data. [S.1.], 2006. p. 281-286. Citations on pages 49 and 67.

ESTER, M.; KRIEGEL, H.-P.; SANDER, J.; XU, X. A density-based algorithm for discovering clusters a density-based algorithm for discovering clusters in large spatial databases with noise. In: Proceedings of the Second International Conference on Knowledge Discovery and Data Mining. AAAI Press, 1996. (KDD'96), p. 226-231. Available: <http://dl.acm.org/citation.cfm? $\mathrm{id}=3001460.3001507>$. Citation on page 45 . 
FACELI, K.; LORENA, A.; GAMA, J.; CARVALHO, A. Inteligência artificial: uma abordagem de aprendizado de máquina. [S.1.]: GEN-LTC. Ltda., 2011. Citations on pages 27 and 44 .

FALOUTSOS, M.; FALOUTSOS, P.; FALOUTSOS, C. On power-law relationships of the internet topology. SIGCOMM Comput. Commun. Rev., ACM, New York, NY, USA, v. 29, n. 4, p. 251-262, 1999. ISSN 0146-4833. Citation on page 35.

FILIPPONE, M.; CAMASTRA, F.; MASULLI, F.; ROVETTA, S. A survey of kernel and spectral methods for clustering. Pattern recognition, Elsevier, v. 41, n. 1, p. 176-190, 2008. Citation on page 46 .

FORNÉS, A.; LLADÓS, J.; SÁNCHEZ, G. Old Handwritten Musical Symbol Classification by a Dynamic Time Warping Based Method, Graphics Recognition. Recent Advances and New Opportunities: 7th International Workshop, GREC 2007, Curitiba, Brazil, September 20-21, 2007. Selected Papers. [S.1.]: Springer-Verlag, Berlin, Heidelberg, 2008. Citation on page 72 .

FORTUNATO, S. Community detection in graphs. Physics reports, Elsevier, v. 486, n. 3-5, p. 75-174, 2010. Citation on page 33.

FOWLKES, C. L. M. E. B. A method for comparing two hierarchical clusterings. Journal of the American Statistical Association, [American Statistical Association, Taylor \& Francis, Ltd.], v. 78, n. 383, p. 553-569, 1983. ISSN 01621459. Available: <http://www.jstor.org/stable/ $2288117>$. Citation on page 42.

FRALEY, C.; RAFTERY, A. E. How many clusters? which clustering method? answers via model-based cluster analysis. The computer journal, Br Computer Soc, v. 41, n. 8, p. 578-588, 1998. Citations on pages 43, 45, and 47.

Mclust: Software for model-based cluster analysis. Journal of Classification, Springer, v. 16, n. 2, p. 297-306, 1999. Citation on page 51.

Model-based clustering, discriminant analysis, and density estimation. Journal of the American statistical Association, Taylor \& Francis, v. 97, n. 458, p. 611-631, 2002. Citation on page 45 .

FRITZKE, B. Kohonen feature maps and growing cell structures-a performance comparison. In: Advances in neural information processing systems. [S.1.: s.n.], 1993. p. 123-130. Citation on page 38 .

GAN, G.; MA, C.; WU, J. Data clustering: theory, algorithms, and applications. [S.1.]: SIAM, 2007. Citation on page 46.

GARCÍA-ROIS, J.; BURGUILLO, J. C. Topology-based analysis of self-organizing maps for time series prediction. Soft Computing, Springer, v. 21, n. 6, p. 1601-1618, 2017. Citation on page 27 .

GUHA, S.; RASTOGI, R.; SHIM", K. Cure: an efficient clustering algorithm for large databases". Information Systems, v. 26, n. 1, p. 35 - 58, 2001. ISSN "0306-4379". Available: <"http: //www.sciencedirect.com/science/article/pii/S0306437901000084">. Citation on page 42. 
HALKIDI, M.; BATISTAKIS, Y.; VAZIRGIANNIS, M. On clustering validation techniques. Journal of intelligent information systems, Springer, v. 17, n. 2-3, p. 107-145, 2001. Citation on page 42 .

HAN, J.; KAMBER, M. Data Mining. Concepts and Techniques. 2nd ed.. ed. -: Morgan Kaufmann, 2006. ISBN 1558609016. Citation on page 45.

HAN, J.; PEI, J.; KAMBER, M. Data mining: concepts and techniques. [S.1.]: Elsevier, 2011. Citation on page 44.

HARTIGAN, J. A.; WONG, M. A. A k-means clustering algorithm. JSTOR: Applied Statistics, v. 28, n. 1, p. 100-108, 1979. Citation on page 51.

HEYLIGHEN, F. et al. The science of self-organization and adaptivity. The encyclopedia of life support systems, v. 5, n. 3, p. 253-280, 2001. Citation on page 78.

HILGETAG, C.-C.; BURNS, G. A.; O’NEILL, M. A.; SCANNELL, J. W.; YOUNG, M. P. Anatomical connectivity defines the organization of clusters of cortical areas in the macaque and the cat. Philosophical Transactions of the Royal Society of London B: Biological Sciences, The Royal Society, v. 355, n. 1393, p. 91-110, 2000. Citation on page 84.

HIRSCHBERGER, M.; QI, Y.; STEUER, R. E. Randomly generating portfolio-selection covariance matrices with specified distributional characteristics. European Journal of Operational Research, Elsevier, v. 177, n. 3, p. 1610-1625, 2007. Citation on page 51.

HOPFIELD, J. Neural networks and physical systems with emergent collective computational abilities. Proceedings of the National Academy of Sciences, National Acad Sciences, v. 79, n. 8 , p. 2554-2558, 1982. Citation on page 26.

HORN, R. A.; JOHNSON, C. R. Matrix Analysis. 2nd. ed. New York, NY, USA: Cambridge University Press, 2012. ISBN 0521548233, 9780521548236. Citation on page 51.

HOWE, D.; COSTANZO, M.; FEY, P.; GOJOBORI, T.; HANNICK, L.; HIDE, W.; HILL, D.; KANIA, R.; SCHAEFFER, M.; PIERRE, S. S. et al. Big data: The future of biocuration. Nature, Nature Publishing Group, v. 455, n. 7209, p. 47-50, 2008. Citation on page 27.

HU, W.; XIE, D.; TAN, T.; MAYBANK, S. Learning activity patterns using fuzzy self-organizing neural network. IEEE Transactions on Systems, Man, and Cybernetics, Part B (Cybernetics), IEEE, v. 34, n. 3, p. 1618-1626, 2004. Citation on page 38.

HUANG, J.; SUN, H.; KANG, J.; QI, J.; DENG, H.; SONG, Q. Esc: An efficient synchronizationbased clustering algorithm. Knowledge-Based Systems, Elsevier, v. 40, p. 111-122, 2013. Citation on page 42 .

HUANG, Z. Extensions to the k-means algorithm for clustering large data sets with categorical values. Data mining and knowledge discovery, Springer, v. 2, n. 3, p. 283-304, 1998. Citation on page 44.

JACCARD, P. Nouvelles recherches sur la distribution florale. Bulletin de la Sociète Vaudense des Sciences Naturelles, v. 44, p. 223-270, 1908. Citation on page 42.

JAIN, A. Data clustering: 50 years beyond k-means. Pattern Recognition Letters, Elsevier, v. 31, n. 8, p. 651-666, 2010. Citation on page 44. 
JAIN, A.; DUBES, R. Algorithms for clustering data. [S.1.]: Prentice-Hall, Inc., 1988. Citations on pages 41 and 46 .

JAIN, A. K.; MURTY, M. N.; FLYNN, P. J. Data clustering: a review. ACM computing surveys (CSUR), Acm, v. 31, n. 3, p. 264-323, 1999. Citation on page 44.

JAIN, A. K.; TOPCHY, A.; LAW, M. H.; BUHMANN, J. M. Landscape of clustering algorithms. In: IEEE. Pattern Recognition, 2004. ICPR 2004. Proceedings of the 17th International Conference on. [S.1.], 2004. v. 1, p. 260-263. Citations on pages 43 and 44.

Jiang, F.; Berry, H.; Schoenauer, M. Optimising the topology of complex neural networks. ArXiv e-prints, oct 2007. Citation on page 27.

JUNG, Y. G.; KANG, M. S.; HEO, J. Clustering performance comparison using k-means and expectation maximization algorithms. Biotechnology \& Biotechnological Equipment, Taylor \& Francis, v. 28, n. sup1, p. S44-S48, 2014. Citations on pages 47 and 49.

KARATZOGLOU, A.; SMOLA, A.; HORNIK, K.; ZEILEIS, A. kernlab-an s4 package for kernel methods in $r$. Institut für Statistik und Mathematik, WU Vienna University of Economics and Business, 2004. Citation on page 51.

KASKI, S.; HONKELA, T.; LAGUS, K.; KOHONEN, T. Websom-self-organizing maps of document collections1. Neurocomputing, Elsevier, v. 21, n. 1-3, p. 101-117, 1998. Citation on page 38 .

KAUFMAN, L.; ROUSSEEUW, P. J. Finding Groups in Data: An Introduction to Cluster Analysis. [S.1.]: John Wiley, 1990. Citations on pages 44 and 51.

KELSO, J. S.; OPSTAL, A. v. Dynamic patterns: the self-organization of brain and behavior. Journal of Cognitive Neuroscience, Cambridge, Mass.: Published by the MIT Press with the Cognitive Neuroscience Institute, c1989-, v. 8, n. 4, p. 385, 1996. Citation on page 78.

KINNUNEN, T.; SIDOROFF, I.; TUONONEN, M.; FRÄNTI, P. Comparison of clustering methods: A case study of text-independent speaker modeling. Pattern Recognition Letters, Elsevier, v. 32, n. 13, p. 1604-1617, 2011. Citations on pages 47 and 49.

KLEMM, K.; EGUÍLUZ, V. M. Growing scale-free networks with small-world behavior. Physical Review E, APS, v. 65, n. 5, p. 057102, 2002. Citations on pages 15, 35, and 36.

KOHONEN, T. Self-organized formation of topologically correct feature maps. Biological cybernetics, Springer, v. 43, n. 1, p. 59-69, 1982. Citations on pages 25 and 38.

Essentials of the self-organizing map. Neural Networks, Elsevier, v. 37, p. 52-65, 2013. Citations on pages 25 and 39.

KOU, G.; PENG, Y.; WANG, G. Evaluation of clustering algorithms for financial risk analysis using mcdm methods. Information Sciences, Elsevier, v. 275, p. 1-12, 2014. Citation on page 49.

KRIEGEL, H.-P.; KRÖGER, P.; ZIMEK, A. Subspace clustering. Wiley Interdisciplinary Reviews: Data Mining and Knowledge Discovery, Wiley Online Library, v. 2, n. 4, p. 351364, 2012. Citation on page 46. 
LAWRENCE, H.; ARABIE, P. Comparing partitions. Journal of Classification, v. 2, n. 1, p. 193-218, 1985. ISSN 1432-1343. Citation on page 42.

LECUN, Y. The mnist database of handwritten digits. http://yann. lecun. com/exdb/mnist/, 1998. Citation on page 72.

LI, Z.; ZHANG, S.; WANG, R.-S.; ZHANG, X.-S.; CHEN, L. Quantitative function for community detection. Physical review E, APS, v. 77, n. 3, p. 036109, 2008. Citation on page 33.

LIU, Y.-C.; WU, C.; LIU, M. Research of fast som clustering for text information. Expert Systems with Applications, Elsevier, v. 38, n. 8, p. 9325-9333, 2011. Citation on page 38.

LUXBURG, U. V. A tutorial on spectral clustering. Statistics and computing, Springer, v. 17, n. 4, p. 395-416, 2007. Citation on page 46.

MACQUEEN, J. et al. Some methods for classification and analysis of multivariate observations. In: OAKLAND, CA, USA. Proceedings of the fifth Berkeley symposium on mathematical statistics and probability. [S.1.], 1967. v. 1, n. 14, p. 281-297. Citations on pages 44 and 50.

MAJUMDER, A.; BEHERA, L.; SUBRAMANIAN, V. K. Emotion recognition from geometric facial features using self-organizing map. Pattern Recognition, Elsevier, v. 47, n. 3, p. 12821293, 2014. Citation on page 39.

MANGIAMELI, P.; CHEN, S. K.; WEST, D. A comparison of som neural network and hierarchical clustering methods. European Journal of Operational Research, Elsevier, v. 93, n. 2, p. 402-417, 1996. Citation on page 50.

MAULIK, U.; BANDYOPADHYAY, S. Performance evaluation of some clustering algorithms and validity indices. IEEE Transactions on Pattern Analysis and Machine Intelligence, IEEE, v. 24, n. 12, p. 1650-1654, 2002. Citations on pages 47 and 48.

MCCULLOCH, W. S.; PITTS, W. A logical calculus of the ideas immanent in nervous activity. The bulletin of mathematical biophysics, Springer, v. 5, n. 4, p. 115-133, 1943. Citation on page 38 .

MCGRAW, P. N.; MENZINGER, M. Topology and computational performance of attractor neural networks. Physical Review E, APS, v. 68, n. 4, p. 047102, 2003. Citation on page 26.

MCKIGHT, P. E.; NAJAB, J. Kruskal-wallis test. Corsini Encyclopedia of Psychology, Wiley Online Library, 2010. Citation on page 58.

MINGOTI, S. A.; LIMA, J. O. Comparing som neural network with fuzzy c-means, k-means and traditional hierarchical clustering algorithms. European Journal of Operational Research, Elsevier, v. 174, n. 3, p. 1742-1759, 2006. Citation on page 50.

MO, H.; XU, B.; OUYANG, W.; WANG, J. Color segmentation of multi-colored fabrics using self-organizing-map based clustering algorithm. Textile Research Journal, SAGE Publications Sage UK: London, England, v. 87, n. 3, p. 369-380, 2017. Citation on page 27.

MURAKAMI, M.; ISHIKURA, S.; KOMINAMI, D.; SHIMOKAWA, T.; MURATA, M. Robustness and efficiency in interconnected networks with changes in network assortativity. Applied Network Science, v. 2, n. 1, p. 6, Mar 2017. ISSN 2364-8228. Available: $<$ https://doi.org/10.1007/s41109-017-0025-4>. Citation on page 36. 
NASCIMENTO, M. C.; CARVALHO, A. C. D. Spectral methods for graph clustering-a survey. European Journal of Operational Research, Elsevier, v. 211, n. 2, p. 221-231, 2011. Citation on page 45 .

NEWMAN, M. Networks: an introduction. [S.1.]: Oxford University Press, 2010. Citations on pages 31 and 34 .

Networks: an introduction. [S.1.]: Oxford university press, 2010. Citation on page 71.

NEWMAN, M. E. The structure and function of complex networks. SIAM review, SIAM, v. 45, n. 2, p. 167-256, 2003. Citation on page 34.

Modularity and community structure in networks. Proceedings of the national academy of sciences, National Acad Sciences, v. 103, n. 23, p. 8577-8582, 2006. Citation on page 33.

NEWMAN, M. E.; GIRVAN, M. Finding and evaluating community structure in networks. Physical review E, APS, v. 69, n. 2, p. 026113, 2004. Citation on page 33.

NEWMAN, M. E. J. Mixing patterns in networks. Phys. Rev. E, American Physical Society, v. 67, n. 2, p. 026126, Feb. 2003. Citations on pages 37 and 71.

ORTIZ, A.; GORRIZ, J.; RAMIREZ, J.; SALAS-GONZALEZ, D. Improving mr brain image segmentation using self-organising maps and entropy-gradient clustering. Information Sciences, Elsevier, v. 262, p. 117-136, 2014. Citation on page 38.

PALLA, G.; BARABÁSI, A.-L.; VICSEK, T. Quantifying social group evolution. Nature, Nature Publishing Group, v. 446, n. 7136, p. 664, 2007. Citation on page 25.

PARSONS, L.; HAQUE, E.; LIU, H. Subspace clustering for high dimensional data: a review. ACM SIGKDD Explorations Newsletter, ACM, v. 6, n. 1, p. 90-105, 2004. Citations on pages 46 and 50 .

PARSONS, L.; HAQUE, E.; LIU, H. et al. Evaluating subspace clustering algorithms. In: CITESEER. Workshop on Clustering High Dimensional Data and its Applications, SIAM Int. Conf. on Data Mining. [S.1.], 2004. p. 48-56. Citation on page 50.

PIRIM, H.; EKŞIOĞLU, B.; PERKINS, A. D.; YÜCEER, Ç. Clustering of high throughput gene expression data. Computers \& operations research, Elsevier, v. 39, n. 12, p. 3046-3061, 2012. Citations on pages 47 and 48 .

R Development Core Team. R: A Language and Environment for Statistical Computing. Vienna, Austria, 2006. ISBN 3-900051-07-0. Available: <http://www.R-project.org>. Citation on page 47.

REDNER, R.; WALKER, H. Mixture densities, maximum likelihood and the em algorithm. SIAM Review, v. 26, n. 6, 1984. Citation on page 45.

REICHMAN, O.; JONES, M.; SCHILDHAUER, M. Challenges and opportunities of open data in ecology. Science(Washington), American Association for the Advancement of Science, 1200 New York Avenue, NW Washington DC 20005 USA, v. 331, n. 6018, p. 703-705, 2011. Citation on page 27.

ROSENBLATT, F. The perceptron: a probabilistic model for information storage and organization in the brain. Psychological review, American Psychological Association, v. 65, n. 6, p. 386, 1958. Citation on page 38. 
RUSSO, R.; HERRMANN, H. J.; ARCANGELIS, L. de. Brain modularity controls the critical behavior of spontaneous activity. Scientific reports, Nature Publishing Group, v. 4, p. 4312, 2014. Citation on page 25.

SAKAR, A.; MAMMONE, R. J. Growing and pruning neural tree networks. IEEE Transactions on Computers, IEEE, v. 42, n. 3, p. 291-299, 1993. Citation on page 38.

SIMARD, D.; NADEAU, L.; KRÖGER, H. Fastest learning in small-world neural networks. Physics Letters A, Elsevier, v. 336, n. 1, p. 8-15, 2005. Citation on page 26.

SOUTO, M. C. de; COSTA, I. G.; ARAUJO, D. S. de; LUDERMIR, T. B.; SCHLIEP, A. Clustering cancer gene expression data: a comparative study. BMC bioinformatics, BioMed Central, v. 9, n. 1, p. 1, 2008. Citations on pages 47 and 49.

STEINLEY, D. K-means clustering: a half-century synthesis. British Journal of Mathematical and Statistical Psychology, Wiley Online Library, v. 59, n. 1, p. 1-34, 2006. Citation on page 44.

STREHL, A.; GHOSH, J.; CARDIE, C. Cluster ensembles - a knowledge reuse framework for combining multiple partitions. Journal of Machine Learning Research, v. 3, p. 583-617, 2002. Citation on page 42 .

SU, M.-C.; CHANG, H.-T. Fast self-organizing feature map algorithm. IEEE Transactions on Neural Networks, IEEE, v. 11, n. 3, p. 721-733, 2000. Citation on page 38.

TASDEMIR, K.; MERÉNYI, E. Exploiting data topology in visualization and clustering of self-organizing maps. IEEE Transactions on Neural Networks, IEEE, v. 20, n. 4, p. 549-562, 2009. Citation on page 25.

TSAO, E. C.-K.; BEZDEK, J. C.; PAL, N. R. Fuzzy kohonen clustering networks. Pattern recognition, Elsevier, v. 27, n. 5, p. 757-764, 1994. Citation on page 38.

VERMA, D.; MEILA, M. A comparison of spectral clustering algorithms. University of Washington Tech Rep UWCSE030501, v. 1, p. 1-18, 2003. Citation on page 48.

WANG, X. F.; CHEN, G. Complex networks: small-world, scale-free and beyond. IEEE circuits and systems magazine, IEEE, v. 3, n. 1, p. 6-20, 2003. Citation on page 32.

WATTS, D. J.; STROGATZ, S. H. Collective dynamics of 'small-world' networks. nature, Nature Publishing Group, v. 393, n. 6684, p. 440, 1998. Citation on page 34.

WHITE, S.; SMYTH, P. A spectral clustering approach to finding communities in graphs. In: SIAM. Proceedings of the $\mathbf{2 0 0 5}$ SIAM international conference on data mining. [S.1.], 2005. p. 274-285. Citation on page 42 .

XULVI-BRUNET, R.; SOKOLOV, I. M. Reshuffling scale-free networks: From random to assortative. Phys. Rev. E, American Physical Society, v. 70, p. 066102, Dec 2004. Available: $<$ https://link.aps.org/doi/10.1103/PhysRevE.70.066102>. Citation on page 37.

YANG, J. som: Self- and super-organising map r package. 2004. Available: <http://CRAN. R-project.org>. Citation on page 51.

ZHANG, D.-Q.; CHEN, S.-C. Clustering incomplete data using kernel-based fuzzy c-means algorithm. Neural processing letters, Springer, v. 18, n. 3, p. 155-162, 2003. Citation on page 42. 
ZHANG, G.; PATUWO, B. E.; HU, M. Y. Forecasting with artificial neural networks:: The state of the art. International journal of forecasting, Elsevier, v. 14, n. 1, p. 35-62, 1998. Citation on page 25 .

ZHANG, G. P. Neural networks for classification: a survey. IEEE Transactions on Systems, Man, and Cybernetics, Part C (Applications and Reviews), IEEE, v. 30, n. 4, p. 451-462, 2000. Citation on page 25. 


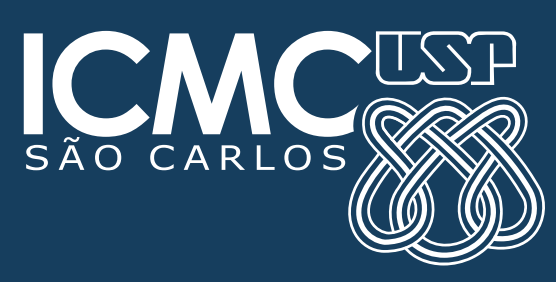

